\title{
Defects in Nematic Shells: a $\Gamma$-convergence discrete-to-continuum approach
}

\author{
Giacomo Canevari · Antonio Segatti
}

Received: date / Accepted: date

\begin{abstract}
In this paper we rigorously investigate the emergence of defects on Nematic Shells with genus different from one. This phenomenon is related to a non trivial interplay between the topology of the shell and the alignment of the director field. To this end, we consider a discrete $X Y$ system on the shell $M$, described by a tangent vector field with unit norm sitting at the vertices of a triangulation of the shell. Defects emerge when we let the mesh size of the triangulation go to zero, namely in the discrete-to-continuum limit. In this paper we investigate the discrete-to-continuum limit in terms of $\Gamma$-convergence in two different asymptotic regimes. The first scaling promotes the appearance of a finite number of defects whose charges are in accordance with the topology of shell $M$, via the Poincaré-Hopf Theorem. The second scaling produces the so called Renormalized Energy that governs the equilibrium of the configurations with defects.
\end{abstract}

Keywords Nematic Shells - XY model - Index of a vector field and Poincaré-Hopf Theorem · Defects · Renormalized Energy $\cdot \Gamma$-convergence

Mathematics Subject Classification (2000) 74K25 - 82B20 - 49J45 . $53 \mathrm{Z} 03 \cdot 76 \mathrm{~A} 15$

\section{Introduction}

Nematic Liquid Crystals offer many intriguing and fascinating examples of a non trivial interplay between topology, geometry, partial differential equations

G. Canevari

BCAM - Basque Center for Applied Mathematics

Alameda de Mazarredo 14, 48009 Bilbao, Vizcaya, Spain. E-mail: gcanevari@bcamath.org

A. Segatti

Dipartimento di Matematica "F. Casorati", Università di Pavia, Via Ferrata 1, 27100 Pavia, Italy. E-mail: antonio.segatti@unipv.it 
and physics (see the recent survey [7]). Interestingly, Liquid Crystals manifest several visual representations of the underlying geometric constraints. For instance, the word Nematic itself originates from the Greek word $\nu \eta \mu \alpha$ (thread) and refers to a particular type of thread-like topological defects that these type of Liquid Crystals exhibit.

In this paper, we are interested in exploring these interplays for Nematic Shells. A Nematic Shell is a rigid colloidal particle with a typical dimension in the micrometer scale coated with a thin film of nematic liquid crystal whose molecular orientation is subjected to a tangential anchoring. The study of these structures has recently received a good deal of interest. As suggested by Nelson [38], the interest in Nematic Shells is related to the possibility of using them as building blocks of mesoatoms with a controllable valence.

From a mathematical point of view, a Nematic Shell is usually represented as a two dimensional compact surface $M$ (without boundary, for simplicity) embedded in $\mathbb{R}^{3}$. As it happens for nematic liquid crystals occupying a domain in $\mathbb{R}^{2}$ or in $\mathbb{R}^{3}$, the basic mathematical description is given in terms of a unitnorm vector field named director, describing the local orientation of the rodshaped molecules of the crystal ([51]). When dealing with nematic shells, the local orientation of the molecules described via a unit-norm tangent vector field $\mathbf{n}: M \rightarrow \mathbb{R}^{3}$ with $\mathbf{n}(x) \in \mathrm{T}_{x} M$ for any $x \in M, \mathrm{~T}_{x} M$ being the tangent plane at the point $x$ ([50], [33], [38], [36], [37], [46], [47]).

The study of these structures offers a non trivial interplay between the geometry and the topology of the fixed substrate and the tangential anchoring constraint. Indeed, as observed in [52] and [14], the liquid crystal equilibrium (and all its stable configurations, in general) is the result of the competition between two driving principles: on the one hand the minimization of the "curvature of the texture" penalized by the elastic energy, and on the other the frustration due to constraints of geometrical and topological nature, imposed by anchoring the nematic to the surface of the underlying particle.

Moreover, the interaction between the local orientation of the molecules and the topology of the surface $M$ (and possibly of the boundary conditions, if any) can induce the formation of topological defects, that is regions of rapid changes in the director field $\mathbf{n}$ (see the survey [8] for detailed references and recent analytical results). It is important to note that point of defects play the role of hot spots for the formation of the mesoatoms suggested by Nelson in [38]. Thus understanding the formation and, possibly, the energetics of defect configurations is extremely significant for applications. This type of problems have been already discussed in the physics community, see e.g. [53] and [14] and references therein.

When dealing with smooth vector fields, the classical Poincaré-Hopf Theorem establishes a link between the existence of a continuous tangent vector field with unit norm on a surface $M$ and the topology of the surface itself. To have a clue on what happens for Nematic Shells, let us consider the simplest 
form of the energy (see [50], [33], [38]):

$$
E(\mathbf{v}):=\frac{1}{2} \int_{M}|\mathrm{D} \mathbf{v}|^{2} \mathrm{~d} S,
$$

where $\mathrm{D}$ is the covariant derivative on $M$. It turns out that the rigorous analysis of nematic shells has to face with possible weak forms of the Poincaré-Hopf Theorem. In particular, introducing the "Sobolev set"

$$
\begin{gathered}
W_{\mathrm{tan}}^{1,2}\left(M ; \mathbb{S}^{2}\right):=\left\{\mathbf{v}: M \rightarrow \mathbb{R}^{3},|\mathbf{v}(x)|=1, \mathbf{v}(x) \in \mathrm{T}_{x} M \text { for a.a. } x \in M,\right. \\
\left.|\mathrm{D} \mathbf{v}| \in L^{2}(M)\right\},
\end{gathered}
$$

we have that (see [47] and [18])

$$
W_{\mathrm{tan}}^{1,2}\left(M ; \mathbb{S}^{2}\right) \neq \emptyset \Leftrightarrow \chi(M)=0
$$

where $\chi(M)$ is the Euler Characteristic of $M$. Consequently, the emergence of defects is exactly related to the choice of the topology of $M$ via the Euler characteristic. More in detail (see [18]), the precise relation between the topology of the surface and the topological charge of the defects is given by the Poincaré-Hopf Theorem: If the unit-norm vector field $\mathbf{v}$ on $M$ has singularities of degree $d_{i}$ located at the point $x_{i}$ for $i=1, \ldots, k$, then

$$
\sum_{i=1}^{k} d_{i}=\chi(M)
$$

The goal of this paper is to understand the emergence of defects for shells of genus different from one (that is, non zero Euler Characteristic) and their energetics. The defect generation is related to the impossibility, for shells with $\chi \neq 0$, of supporting a tangent, unit-norm vector field with the Sobolev regularity above. Thus, a possibile strategy would be to relax one the above constraints, for instance the unit-norm constraint as in the Ginzburg-Landau theory. On a Euclidean domain $\Omega \subseteq \mathbb{R}^{d}, d \geq 2$, the Ginzburg-Landau functional reads

$$
E_{\varepsilon}^{\mathrm{GL}}(u):=\int_{\Omega}\left\{\frac{1}{2}|\nabla u|^{2}+\frac{1}{4 \varepsilon^{2}}\left(1-|u|^{2}\right)^{2}\right\}
$$

and is defined for $u \in W^{1,2}(\Omega, \mathbb{C}), \varepsilon>0$ being a small parameter. The asymptotic behaviour of Ginzburg-Landau minimizers as $\varepsilon \rightarrow 0$, in case $\Omega$ is a Euclidean domain, has been extensively addressed (see, for instance, [12], [27], [40], [29], [41]). Ginzburg-Landau functionals for complex-valued maps defined on a surface have been considered by Baraket [9], who studied the asymptotic behaviour of minimizers in the singular limit $\varepsilon \rightarrow 0$, and by Contreras and Sternberg [20], who worked for fixed $\varepsilon>0$ and derived the model as a thin-film limit of the three-dimensional Ginzburg-Landau functional, via $\Gamma$-convergence. Qing [39] studied a Ginzburg-Landau functional depending on $(u, A)$, where $u$ is a section of an arbitrary line bundle on a closed Riemann surface $M$ (in particular, $u$ may be a tangent field on $M$ ) and $A$ is a 
connection on that bundle; the functional in [39] includes a dependence on the curvature of $A$. Recently, Ignat and Jerrard [25] studied the singular limit of a Ginzburg-Landau energy for tangent vector fields on a closed surface, in the framework of $\Gamma$-convergence.

In this paper, we choose another point of view and instead of a continuous model we rather consider a discrete one with the molecules sitting at the vertices of a triangular mesh approximating the surface $M$. One of the advantages of this approach is that it paves the way for a computational analysis in terms of finite elements.

The model we consider here is a variant of the well-known $X Y$-spin model, which is widely regarded as a prototypical example of a discrete spin system where phase transitions that are mediated by topological defects occur. Such phase transitions were first identified by Kosterlitz and Thouless [31] (also based on previous work by Berezinskii [11]), who were awarded the 2016 Nobel Prize for Physics, together with Haldane, in recognition of their discoveries on topological phases of matter. $X Y$-models have also attracted attention in the mathematical community; see, for instance, [1], [2], [16], where the discrete-to-continuum limit of such models, and their connection with the continuum Ginzburg-Landau theory, is explored. The aforementioned papers are concerned with the study of "flat" situations, i.e. the model is set on a domain $\Omega \subseteq \mathbb{R}^{2}$; the dynamics for an $X Y$-model on a "curved" torus has been numerically explored e.g. in [48], via a Monte-Carlo approach.

In this paper, we aim to address the mathematical analysis of an $X Y$-model on surfaces. More precisely, given a closed surface $M \subseteq \mathbb{R}^{3}$ with $\chi(M) \neq 0$, we consider a family of triangulations $\mathcal{T}_{\varepsilon}$ of $M$ with the vertices $i \in \mathcal{T}_{\varepsilon}^{0}$ lying on $M$ and with mesh size $\varepsilon$, i.e. $\varepsilon=\max _{T \in \mathcal{T}_{\varepsilon}} \operatorname{diam}(T)$ (see Section 3 for the details). Any point $i \in \mathcal{T}_{\varepsilon}^{0}$ is occupied by a unit-norm tangent vector $\mathbf{v}_{\varepsilon}(i) \in \mathrm{T}_{i} M$. Our energy functional takes the form

$$
X Y_{\varepsilon}\left(\mathbf{v}_{\varepsilon}\right):=\frac{1}{2} \sum_{i \neq j \in \mathcal{T}_{\varepsilon}^{0}} \kappa_{\varepsilon}^{i j}\left|\mathbf{v}_{\varepsilon}(i)-\mathbf{v}_{\varepsilon}(j)\right|^{2},
$$

where the coefficients $\kappa_{\varepsilon}^{i j}$ are the entries of the stiffness matrix of $M$, that is, the finite-element discretization of the Laplace-Beltrami operator $\left(\right.$ see $\left(\mathrm{H}_{3}\right)$ for the definition). Our discrete energy is effectively induced by nearest-neighbours interactions, as the coefficient $\kappa_{\varepsilon}^{i j}=0$ unless $i, j$ are adjacent vertices.

As we will see later on in this paper, the $X Y_{\varepsilon}$ energy is intimately related to the Dirichlet energy of the piece wise interpolation of the discrete vectors $\mathbf{v}_{\varepsilon}(i)$. Consequently, when $\varepsilon \rightarrow 0$ we expect that the behavior of $X Y_{\varepsilon}$ is dictated by (2), and a uniform bound on the energy of a minimizer can hold if and only if the Euler Characteristic is equal to zero. It is important to note that the discrete (tangent) vectors $\mathbf{v}(i)$ are identified with tangent vectors in $\mathbb{R}^{3}$ and thus the difference appearing in the definition of the energy is exactly a difference between vectors in $\mathbb{R}^{3}$. When $\chi(M)=0$, this fact brings important consequences in the distorsion effect in the limit energy (see Proposition 1) and in particular produces a macroscopic energy that is capable of describing also the extrinsic effects. 
When $\chi(M) \neq 0$, configurations with defects emerge when we let $\varepsilon \rightarrow 0$, namely in the discrete-to-continuum limit. Thus, the very goal of this paper is to analyze this limit in terms of $\Gamma$-convergence, in the spirit of [1], [2]. As it is typical in $\Gamma$-convergence results, one obtains possibly different limits according to the chosen scaling of the energy.

Our first main result (see Theorem A) exactly relates the emergence of defects with the topology of the shell $M$ and is expressed in terms of the $\Gamma$ convergence (in a suitable topology) of $\frac{X Y_{\varepsilon}(\cdot)}{|\log \varepsilon|}$. Following the flat case (see [29], [1], [2]), we introduce the so-called vorticity measure $\widehat{\mu}_{\varepsilon}\left(\mathbf{v}_{\varepsilon}\right)$ of a discrete field $\mathbf{v}_{\varepsilon}$, which is a kind of discrete notion of the Jacobian. This quantity captures all the relevant "topological information" of $\mathbf{v}_{\varepsilon}$. For sequences $\left(\mathbf{v}_{\varepsilon}\right)_{\varepsilon}$ that satisfy a logarithmic energy bound (e.g., minimizers), we show that $\widehat{\mu}_{\varepsilon}\left(\mathbf{v}_{\varepsilon}\right)$ converges, in a suitable topology, to a measure of the form $2 \pi \sum_{i=1}^{\mathscr{K}} d_{i} \delta_{x_{i}}-G \mathrm{~d} S$, where the points $x_{i} \in M$ correspond to the position of the defects, the coefficients $d_{i} \in \mathbb{Z}$ are the topological charges, $G$ is the Gauss curvature and $\mathrm{d} S$ is the area element on $M$. The proof follows the steps of analogous results in the Ginzburg-Landau literature (see, in particular, [29], [27] for the continuous setting and [1], [2] for the discrete $X Y$-setting).

Our second main result (see Theorem B) is on the energetics of configurations with defects. More precisely, we investigate the $\Gamma$-convergence of $X Y_{\varepsilon}(\cdot)-\pi \mathscr{K}|\log \varepsilon|$, where $\mathscr{K}$ is the total variation of the measure $\sum_{i=1}^{\mathscr{K}} d_{i} \delta_{x_{i}}$ when $\left|d_{i}\right|=1$ for all $i=1, \ldots, \mathscr{K}$. What emerges in the $\Gamma$-limit is the so called Renormalized Energy $\mathbb{W}$ that has been first studied in a rigorous way for the Ginzburg-Landau theory by Brezis, Bethuel and Hélein in [12]. The literature on this topic is very vast (see [3] and references therein) and includes also results for the discrete $X Y$ model on the plane, see [2]. In the euclidean situation this energy depends on the position of the singularities via the Green function of the laplacian on $\mathbb{R}^{2}$. When dealing with a curved substrate $M$, one may expects that the curvature properties of $M$ intervene in the limit (differently from the zeroth order $\Gamma$-convergence in which only the topological properties of $M$ come into play). In particular, we expect that the curvature of $M$ (more precisely the Gaussian curvature) enters in the expression of the Renormalized Energy and acts as a further geometric driving principle in the location of defects. This intuition is indeed true, as the analysis in [53] shows for a corrugated plane, that is the graph of a gaussian function. For this specific surface Vitelli and Nelson in [53] show that the Renormalized Energy is given by a sum of different terms including the Green function of the Laplace Beltrami on the surface and contributions coming from the Gaussian curvature of $M$. Interestingly, Vitelli and Nelson exhibit a term in the Renormalized energy given by interaction between the charge of the defects and the curvature of $M$. Instead, our Theorem B deals with a compact surface $M$ and rigorously prove the $\Gamma$-convergence of $X Y_{\varepsilon}(\cdot)-\pi \mathscr{K}|\log \varepsilon|$, to a Renormalized Energy that takes into account also how $M$ sits in the three dimensional space. In fact, $\mathbb{W}$ is given by the sum of a purely intrinsic part and and extrinsic part related with the shape operator of $M$. 
As a first consequence of our result, we have the following asymptotic expansion for the minima of $X Y_{\varepsilon}$ (thanks to classical results in $\Gamma$-convergence):

$$
\min X Y_{\varepsilon}=\pi|\chi(M)||\log \varepsilon|+\mathbb{W}(\mathbf{v})+\sum_{i=1}^{|\chi(M)|} \gamma\left(x_{i}\right)+\mathrm{o}_{\varepsilon \rightarrow 0}(1),
$$

where $\mathbf{v} \in W_{\text {tan,loc }}^{1,2}\left(M \backslash\left\{x_{1}, \ldots, x_{|\chi(M)|}\right\} ; \mathbb{S}^{2}\right)$ is the "continuum limit" of the sequence of discrete minimizers and $\gamma\left(x_{i}\right)$ is a positive quantity that takes into account the energy located in the core of the defects $x_{i}$ of $\mathbf{v}$. It is interesting to note that $\sum_{i=1}^{|\chi(M)|} \gamma\left(x_{i}\right)$ has memory of the discrete structure around any singularity $x_{i}$ in the sense that $\gamma\left(x_{i}\right)$ will depend on the (limit) triangulation around each defect $x_{i}$. This is a purely discrete phenomenon which is essentially due to the fact that for a curved shell the vertices of the triangulation do not necessarily sit on a structured lattice. Another striking difference from the planar case, both continuous and discrete (see [12] and [2]), and from the curved continuous case (see [25]), is that the core energy $\gamma\left(x_{i}\right)$ depends on the singularity $x_{i}$.

It is important to note that the Renormalized energy we introduce in this paper is defined on a proper class of vector fields (see $(17),(18)$ in the present paper and [2, Eq. 4.21]). In particular, our choice is different from the classical choice of Brezis, Bethuel and Hélein [12] that rather define the Renormalized Energy as a functional of the configuration and of the charge of the singularities. However, the two definitions are indeed intimately linked (at least on the planar case, see [2, Eq. 4.23], and for the intrinsic part of the Rernomalized Energy, see (21)) by a minimization procedure. For a general surface $M$, the form of the Renormalized Energy $\mathbb{W}$ is rather implicit and, as expected, one should work down its expression for any given surface $M$. In analogy with the well established planar case and guided by the computation in [53] for the corrugated plane, we expect in particular that the Renormalized energy should comprise a term related with the Green function of the Laplace Beltrami operator on $M$ whose explicit expression heavily depends on the form of $M$.

Even if our analysis was motivated by Nematic Shells, the study of the interplay between the topological properties of the domain and the possible formation of singularities with infinite energy, is common to other models as the emergence of (topological) defects is ubiquitous in nature (see [30] and references therein). In particular, their topological origin often give the system configurations exhibiting defects a universal feature. Consequently, the issues we aim at addressing have a more general flavor and are independent of the particular system. Moreover, energy functionals such as (1) are commonly used also to model Amphiphilic molecules exhibiting an hexatic bond orientational order ([35], [15]). Thus, the horizon of our analysis and results is much wider than just Nematic Shells.

During the preparation of the present paper, we were informed that $\mathrm{R}$. Ignat and R. Jerrard (see [25] and [26]) were obtaining, independently from us, 
similar results (among the others, the Renormalized Energy) for a GinzburgLandau type functional on a two dimensional surface $M \subseteq \mathbb{R}^{3}$.

\section{Differential geometry notation}

In this section we briefly introduce the differential geometry formalism that we use in the paper. We refer to [23] and [32] for basic references on this subject.

Let $M \subseteq \mathbb{R}^{3}$ be a smooth, compact, connected surface without boundary, oriented by the choice of a smooth, unit normal field $\gamma: M \rightarrow \mathbb{R}^{3}$. We denote by $\boldsymbol{\omega}$ the volume form induced by this choice of the orientation and by $\mathrm{d} S$ the area element on $M$, i.e., the positive Borel measure induced by $\boldsymbol{\omega}$. We let $G$ be the Gauss curvature of $M$. By abuse of notation, we identify $G$ with the Borel measure $G \mathrm{~d} S$ and, if no confusion is possible, we write $G$ in place of $G \mathrm{~d} S$. For any point $x \in M$, we let $\mathrm{T}_{x} M$ denote the tangent plane at $x$ and we dnote with $\mathrm{T} M$ the tangent bundle of $M$, namely the disjoint union $\bigsqcup_{x \in M} \mathrm{~T}_{x} M$. We denote with $\pi: \mathrm{T} M \rightarrow M$ the smooth map that assigns to any tangent vector its application point. A vector field $\mathbf{v}$ on an open neighbourhood of $A \subset M$ is a section of TM, namely a map $\mathbf{v}: A \rightarrow \mathrm{T} M$ for which $\pi \circ \mathbf{v}$ is the identity on $M$. Let $U \subseteq \mathbb{R}^{3}$ be an open tubular neighbourhood of $M$ of thickness $h$ such that

$$
h \leq \min _{x \in M}\left(\max \left(\left|\kappa_{1}(x)\right|,\left|\kappa_{2}(x)\right|\right)\right)^{-1}
$$

where $\kappa_{1}$ and $\kappa_{2}$ are the two principal curvatures of $M$. For a such a tubular neighbourhood the nearest-point projection $P: U \rightarrow M$ is well-defined and smooth.

Let $\nabla$ be the connection with respect to the standard metric of $\mathbb{R}^{3}$, i.e., given two smooth vector fields $Y$ and $X$ in $\mathbb{R}^{3}$ (identified with its tangent space), $\nabla_{X} Y$ is the vector field whose components are the directional derivatives of the components of $Y$ in the direction $X$. We denote with $\mathrm{D}_{\mathbf{v}} \mathbf{u}$ the covariant derivative of $\mathbf{u}$ in the direction $\mathbf{v}$ ( $\mathbf{u}$ and $\mathbf{v}$ are smooth tangent vector fields in $M$ ), with respect to the Levi Civita (or Riemannian) connection D of the metric $g$ on $M$.

Now, if $\mathbf{u}$ and $\mathbf{v}$ are extended arbitrarily to smooth vector fields on $\mathbb{R}^{3}$, we have the Gauss Formula :

$$
\nabla_{\mathbf{v}} \mathbf{u}=\mathrm{D}_{\mathbf{v}} \mathbf{u}+\langle\mathrm{d} \boldsymbol{\gamma}[\mathbf{u}], \mathbf{v}\rangle \boldsymbol{\gamma}
$$

This decomposition is orthogonal, thus there holds

$$
|\nabla \mathbf{u}|^{2}=|\mathrm{D} \mathbf{u}|^{2}+|\mathrm{d} \boldsymbol{\gamma}[\mathbf{u}]|^{2}
$$

Beside the covariant derivative, we introduce another differential operator for vector fields on $M$, which takes into account also the way that $\Sigma$ embeds in $\mathbb{R}^{3}$. Let $\mathbf{u}$ be a smooth vector field on $M$. We extend it smoothly to a vector field $\tilde{\mathbf{u}}$ on $\mathbb{R}^{3}$ and we denote its standard gradient by $\nabla \tilde{\mathbf{u}}$ on $\mathbb{R}^{3}$. For $x \in M$, we define

$$
\nabla_{\mathrm{s}} \mathbf{u}(x):=\nabla \tilde{\mathbf{u}}(x) P_{M}(x)
$$


where $P_{M}(x):=(\operatorname{Id}-\gamma \otimes \gamma)(x)$ is the orthogonal projection on $\mathrm{T}_{x} M$. In other words, $\nabla_{\mathrm{s}}$ is the restriction of the standard derivative in $\mathbb{R}^{3}$ to directions that are tangent to $M$. Note that $\nabla_{\mathrm{s}} \mathbf{u}$ is well-defined, as it does not depend on the particular extension $\tilde{\mathbf{u}}$. The object just defined is a smooth mapping $\nabla_{\mathrm{s}} \mathbf{u}: M \rightarrow \mathbb{R}^{3 \times 3}$, or equivalently $\nabla_{\mathrm{s}} \mathbf{u}: M \rightarrow \mathcal{L}\left(\mathbb{R}^{3}, \mathbb{R}^{3}\right)$ (the space of linear continuous operators on $\left.\mathbb{R}^{3}\right)$. In general, $\nabla_{\mathrm{s}} \mathbf{u} \neq \mathrm{Du}=P_{M}(\nabla \mathbf{u})$ since the matrix product is non commutative. Moreover, thanks to (4) and (5) there holds

$$
\left|\nabla_{\mathrm{s}} \mathbf{u}\right|^{2}=|\mathrm{Du}|^{2}+|\mathrm{d} \boldsymbol{\gamma}[\mathbf{u}]|^{2} .
$$

The difference between the covariant (intrinsic) differentiation $\mathrm{D}$ and the (extrinsic) differentiation $\nabla_{\mathrm{s}}$ is evident when considering $M=\mathbb{S}^{1} \times[0,1]$, namely $M$ is the lateral surface of the cylinder with radius one and height one and with the axis on the $z$ axis in $\mathbb{R}^{3}$. Consider the parametrization $\mathbf{x}(\theta, h)=(\cos \theta, \sin \theta, h)$, with $\theta \in[0,2 \pi]$ and $h \in(0,1)$ and the tangent vector field $\mathbf{u}=\frac{\partial \mathbf{x}}{\partial \theta}=(-\sin \theta, \cos \theta, 0)$. It is a standard computation to show that $\mathbf{u}$ is a parallel vector field, that is Du $\equiv 0$. On the other hand, there holds $\mathrm{d} \boldsymbol{\gamma}[\mathbf{u}]=\mathbf{u}$ that gives $($ see $(7)) \nabla_{\mathrm{s}} \mathbf{u} \neq 0$.

Note that, by identifying $\mathbf{u}$ with a map $\mathbf{u}=\left(\mathbf{u}^{1}, \mathbf{u}^{2}, \mathbf{u}^{3}\right): M \rightarrow \mathbb{R}^{3}$, the $k$ th row of the matrix representing $\nabla_{\mathrm{s}} \mathbf{u}$ coincides with the Riemannian gradient (that we still denote with $\nabla_{s}$ ) of $\mathbf{u}^{k}$. In other words, while D is a connection on the tangent bundle $\mathrm{T} M, \nabla_{\mathrm{s}}$ arises naturally as a connection on the trivial bundle $M \times \mathbb{R}^{3}$.

Let $x_{0} \in M$ and let $\mathbf{v}$ be a vector field defined in a neighbourhood of $x_{0}$ which is continuous and satisfies $\mathbf{v}(x) \neq 0$ for any $x$ in a neighbourhood of $x_{0}$ except, possibly, at $x_{0}$. By taking local coordinates $\varphi: B_{\delta} \subseteq \mathbb{R}^{2} \rightarrow$ $M$ with $\varphi(0)=x_{0}$, we can identify $\mathbf{v}$ with a map $\varphi^{*} \mathbf{v}: B_{\delta} \subseteq \mathbb{R}^{2} \rightarrow \mathbb{R}^{2}$, namely $\varphi^{*} \mathbf{v}(z):=\left\langle\mathrm{d} \varphi^{-1}(\varphi(z)), \mathbf{v}(\varphi(z))\right\rangle$ for $z \in B_{\delta}$. We define the local index ind $\left(\mathbf{v}, x_{0}\right)$ as the topological degree of the map

$$
\frac{\varphi^{*} \mathbf{v}}{\left|\varphi^{*} \mathbf{v}\right|}{ }_{\mid \partial B_{\delta}}: \partial B_{\delta} \simeq \mathbb{S}^{1} \rightarrow \mathbb{S}^{1}
$$

It is easily checked that ind $\left(\mathbf{v}, x_{0}\right)$ does not depend on the choice of $\delta$ nor $\varphi$. The index is well-defined even if $\mathbf{v}$ is a field of Sobolev regularity $W^{1,2}$ because the restriction of $\varphi^{*} \mathbf{v}$ to the circle $\partial B_{\delta}$ is continuous for a.e. $\delta$, thanks to Fubini theorem and Sobolev embedding. Given an open set $E \subseteq M$ and a continuous field $\mathbf{v}$ that has finitely many zeros inside $E$, we let ind $(\mathbf{v}, E):=$ $\sum_{x \in E: \mathbf{v}(x)=0} \operatorname{ind}(\mathbf{v}, x)$. By an approximation $\operatorname{argument}$, ind $(\mathbf{v}, E)$ can then be defined for any field $\mathbf{v} \in W^{1,2}$ with $\operatorname{essinf}_{\partial E}|\mathbf{v}|>0$. For further details on the construction and properties of the index, we refer the reader to, e.g., [18].

\section{The discrete model and main results}

In this section we introduce the discrete setting we will use in the rest of the paper and we state our main results. This Section is organized as follows. 
First of all, we will introduce the discretization of the surface $M$. As the mathematical analysis of (1) bears some analogy with the analysis of harmonic maps (see [47]) the discretization of the surface is based on the formalism developed in [10]. Then, we define the starting point of our analysis, namely the discrete energy $\left(\mathrm{XY}_{\varepsilon}\right)$. Finally, we discuss the (simpler) case of defectsfree textures and then we state our Main results on the emergence of defects (Theorem A) and on their energetics (Theorem B). To ease the presentaion, we will briefly introduce two fundamental objects, namely a discrete version of the jacobian and the Renormalized Energy. Their rigorous definitions together with their properties will be postponed in the forthcoming Subsections 4.5 and 6.1 .

\subsection{Triangulations of a surface}

A triangulation is a 2-dimensional simplicial complex, that is, a finite collection $\mathcal{T}$ of non-degenerate affine triangles $T \subseteq \mathbb{R}^{3}$ with the following property: the intersection of any two triangles $T, T^{\prime} \in \mathcal{T}$ is either empty or a common subsimplex of $T, T^{\prime}$. The sets of vertices and edges of $\mathcal{T}$ will be denoted by $\mathcal{T}^{0}, \mathcal{T}^{1}$, respectively. The size of a triangulation $\mathcal{T}$ is the quantity $\max _{T \in \mathcal{T}} \operatorname{diam}(T)$. A triangulation of a surface $M$ is a triangulation $\mathcal{T}$ such that the set $\cup_{T \in \mathcal{T}} T$ is homeomorphic to $M$.

For a fixed $\varepsilon_{0}>0$ and $\varepsilon \in\left(0, \varepsilon_{0}\right]$, we let $\mathcal{T}_{\varepsilon}$ be a triangulation of $M$ of size $\varepsilon$. For our purposes, it is not enough to require that the set $\widehat{M}_{\varepsilon}:=\cup_{T \in \mathcal{T}_{\varepsilon}} T$ be homeomorphic to $M$; throughout the paper, we will assume that the following stronger condition is satisfied. Recall that the nearest-point projection $P$ onto $M$ is well-defined and smooth in an open neighbourhood $U \subseteq M$.

$\left(\mathrm{H}_{1}\right)$ For any $\varepsilon \in\left(0, \varepsilon_{0}\right], \mathcal{T}_{\varepsilon}^{0} \subseteq M, \widehat{M}_{\varepsilon} \subseteq U$ and $\widehat{P}_{\varepsilon}:=P_{\mid \widehat{M}_{\varepsilon}}: \widehat{M}_{\varepsilon} \rightarrow M$ has a Lipschitz inverse. Moreover, there exists an $\varepsilon$-independent constant $\Lambda$ such that $\operatorname{Lip}\left(\widehat{P}_{\varepsilon}\right)+\operatorname{Lip}\left(\widehat{P}_{\varepsilon}^{-1}\right) \leq \Lambda$ for any $\varepsilon$.

This condition is stronger than just requiring $M, \widehat{M}_{\varepsilon}$ to be topologically equivalent. (For instance, for small $\varepsilon>0$ the tetrahedron of vertices $(\sin \varepsilon, 0, \cos \varepsilon)$, $\left(-\frac{1}{2} \sin \varepsilon, \frac{\sqrt{3}}{2} \sin \varepsilon, \cos \varepsilon\right),\left(-\frac{1}{2} \sin \varepsilon,-\frac{\sqrt{3}}{2} \sin \varepsilon, \cos \varepsilon\right),(0,0,1)$ defines a triangulation of the unit sphere which does not satisfy $\left(\mathrm{H}_{1}\right)$.) However, $\left(\mathrm{H}_{1}\right)$ is satisfied in numerically relevant examples.

Given an open set $\Omega \subseteq M_{\varepsilon}$, we define

$$
\Omega_{\varepsilon}:=\bigcup_{T \in \mathcal{T}_{\varepsilon}: P(T) \subseteq \Omega} P(T)
$$

so that $\Omega_{\varepsilon} \subseteq M$. Moreover, we denote with $\partial_{\varepsilon} \Omega$ the discrete boundary of $\Omega$, namely

$$
\partial_{\varepsilon} \Omega:=\partial \Omega_{\varepsilon} \cap \mathcal{T}_{\varepsilon}^{0}
$$

Given a piecewise-smooth function $\mathbf{u}: \widehat{M}_{\varepsilon} \rightarrow \mathbb{R}^{k}$, we denote by $\nabla_{\varepsilon} \mathbf{u}$ the restriction of the derivative $\nabla \mathbf{u}$ to directions that lie in the triangles of $\widehat{M}_{\varepsilon}$. 
In addition to $\left(\mathrm{H}_{1}\right)$, we assume that the family of triangulations $\left(\mathcal{T}_{\varepsilon}\right)$ satisfies the following conditions.

$\left(\mathrm{H}_{2}\right)$ Let $T_{\text {ref }} \subseteq \mathbb{R}^{2}$ be a reference triangle of vertices $(0,0),(1,0)$ and $(0,1)$. There exists a constant $\Lambda>0$ such that, for any $\varepsilon \in\left(0, \varepsilon_{0}\right]$ and any $T \in \mathcal{T}_{\varepsilon}$, the (unique) affine bijection $\phi: T_{\text {ref }} \rightarrow T$ satisfies

$$
\operatorname{Lip}(\phi) \leq \Lambda \varepsilon, \quad \operatorname{Lip}\left(\phi^{-1}\right) \leq \Lambda \varepsilon^{-1}
$$

Here $\operatorname{Lip}(\phi)$ denotes the Lipschitz constant of $\phi, \operatorname{Lip}(\phi):=\sup _{x \neq y} \mid x-$ $\left.y\right|^{-1}|\phi(x)-\phi(y)|$.

$\left(\mathrm{H}_{3}\right)$ For any $\varepsilon \in\left(0, \varepsilon_{0}\right]$ and any $i, j \in \mathcal{T}_{\varepsilon}^{0}$ with $i \neq j$, there holds

$$
\kappa_{\varepsilon}^{i j}:=-\int_{\widehat{M}_{\varepsilon}} \nabla_{\varepsilon} \widehat{\varphi}_{\varepsilon, i} \cdot \nabla_{\varepsilon} \widehat{\varphi}_{\varepsilon, j} \mathrm{~d} S \geq 0,
$$

where the hat function $\widehat{\varphi}_{\varepsilon, i}$ is the unique piecewise-affine, continuous function $\widehat{M}_{\varepsilon} \rightarrow \mathbb{R}$ such that $\widehat{\varphi}_{\varepsilon, i}(j)=\delta_{i j}$ for any $j \in \mathcal{T}_{\varepsilon}^{0}$.

Remark $1\left(\mathrm{H}_{2}\right)$ is equivalent to the following condition: there exists a constant $\Lambda>0$ such that, for any $\varepsilon \in\left(0, \varepsilon_{0}\right]$ and any triangle $T \in \mathcal{T}_{\varepsilon}$, there holds

$$
\Lambda^{-1} \varepsilon \leq \operatorname{diam}(T) \leq \Lambda \varepsilon \quad \text { and } \quad \alpha_{\min }(T) \geq \Lambda^{-1},
$$

where $\alpha_{\min }(T)$ stands for the minimum of the angles of $T$. Meshes that satisfy this condition are called quasi-uniform in the numerical literature. Since the manifold $M$ is compact and smooth, and hence has bounded curvature, $\alpha_{\min }(T) \geq \Lambda^{-1}$ implies that the number of neighbours of a given vertex is uniformly bounded with respect to $\varepsilon$.

Remark 2 A sufficient condition for $\left(\mathrm{H}_{3}\right)$ is the following: for any pair of triangles $T_{1}, T_{2} \in \mathcal{T}_{\varepsilon}$ that share a common edge $e$, let $\alpha_{i}$ be the angle in $T_{i}$ opposite to $e$ (for $i \in\{1,2\}$ ). If $\alpha_{1}+\alpha_{2} \leq \pi$ for every edge $e$ as above, then $\left(\mathrm{H}_{3}\right)$ holds (see e.g. [10, Lemma 1.4.1]). Triangular meshes that satisfy $\left(\mathrm{H}_{3}\right)$ are called weakly acute.

Remark 3 If $\mathcal{T}_{\varepsilon}$ satisfies $\left(\mathrm{H}_{3}\right)$ and if $\widehat{\varphi}, \widehat{\tau} \in C\left(\widehat{M}_{\varepsilon}, \mathbb{R}\right)$ are piecewise-affine functions on the triangles of $\mathcal{T}_{\varepsilon}$, then

$$
\int_{\widehat{M}_{\varepsilon}} \nabla_{\varepsilon} \widehat{\varphi} \cdot \nabla_{\varepsilon} \widehat{\tau} \mathrm{d} S=\sum_{i, j \in \mathcal{T}_{\varepsilon}^{0}} \kappa_{\varepsilon}^{i j}(\widehat{\varphi}(i)-\widehat{\varphi}(j))(\widehat{\tau}(i)-\widehat{\tau}(j)) .
$$

Remark 4 There are algorithmic ways to construct triangulations that are quasi-uniform, weakly acute and satisfy $\left(\mathrm{H}_{1}\right)$, for instance, Delaunay meshes (see e.g. [49], [4]).

Beside Hypotheses $\left(\mathrm{H}_{1}\right)-\left(\mathrm{H}_{3}\right)$, for the validity of Theorem B we will need a refined control on the triangulation $\mathcal{T}_{\varepsilon}$ around the singularities (see Proposition 5 ) in the limit $\varepsilon \rightarrow 0$. At base, we require that our triangulation $\mathcal{T}_{\varepsilon}$ is somehow scale invariant. We express this requirement as follows. Fix $x \in M$ and let $\delta>$ 
0 be smaller than the injectivity radius of $M$. Using geodesic coordinates, $\varphi: B_{\delta} \subseteq \mathrm{T}_{x} M \simeq \mathbb{R}^{2} \rightarrow M$ such that $\varphi(0)=\bar{x}$, we pull $\mathcal{T}_{\varepsilon}$ back and define a triangulation $\overline{\mathcal{T}}_{\varepsilon}$ on $B_{\delta} \subseteq \mathbb{R}^{2}$. (The set of vertices of $\overline{\mathcal{T}}_{\varepsilon}$ is $\varphi^{-1}\left(B_{\delta}(\bar{x}) \cap \mathcal{T}_{\varepsilon}^{0}\right)$; three vertices in $\overline{\mathcal{T}_{\varepsilon}}$ span a triangle in $\overline{\mathcal{T}}_{\varepsilon}$ if and only if their images via $\varphi$ do.) We scale the triangulation $\overline{\mathcal{T}}_{\varepsilon}$ of a factor $1 / \varepsilon$ and define a triangulation on $B_{\delta / \varepsilon} \subseteq \mathbb{R}^{2}$, namely

$$
\mathcal{S}_{\varepsilon}:=\left\{\frac{1}{\varepsilon} T: T \in \overline{\mathcal{T}}_{\varepsilon}\right\} .
$$

Given another triangulation $\mathcal{S}$ on $\mathbb{R}^{2}$, we denote by $\mathcal{S}_{\mid B_{\delta / \varepsilon}}$ the collection of triangles $T \in \mathcal{S}$ such that $T \subseteq B_{\delta / \varepsilon}$. We express the distance between $\mathcal{S}_{\varepsilon}$ and $\mathcal{S}_{\mid B_{\delta / \varepsilon}}$ as

$$
d\left(\mathcal{S}_{\varepsilon}, \mathcal{S}_{\mid B_{\delta / \varepsilon}}\right):=\inf _{\phi} \max _{i \in \mathcal{S}_{\varepsilon}^{0}}|i-\phi(i)|,
$$

where the infimum is taken over all simplicial isomorphisms $\phi$ from $\mathcal{S}_{\varepsilon}$ to $\mathcal{S}_{\mid B_{\delta / \varepsilon}}$ (that is, piecewise-affine maps that preserves the combinatorial structure of the mesh; see Section 4.1 for more details). In addition to $\left(\mathrm{H}_{1}\right),\left(\mathrm{H}_{2}\right)$ and $\left(\mathrm{H}_{3}\right)$, for the validity of Theorem $\mathrm{B}$ we assume that the following condition holds.

$\left(\mathrm{H}_{4}\right)$ For any $x \in M$ there exists a triangulation $\mathcal{S}=\mathcal{S}(x)$ on $\mathbb{R}^{2}$ such that, for any $\delta>0$ smaller than the injectivity radius of $M$, there holds

$$
\lim _{\varepsilon \searrow 0} d\left(\mathcal{S}_{\varepsilon}, \mathcal{S}_{\mid B_{\delta / \varepsilon}}\right)|\log \varepsilon|=0 .
$$

This assumption is only used in the arguments of Section 6.2, and in particular in the proof of Proposition 5.

Remark 5 The construction of a countable sequence of triangulations $\left(\mathcal{T}_{\varepsilon_{k}}\right)_{k \in \mathbb{N}}$ satisfying $\left(\mathrm{H}_{4}\right)$, as well as $\left(\mathrm{H}_{1}\right)-\left(\mathrm{H}_{3}\right)$, is illustrated in Figure 1 , in case $M$ is the sphere of radius $\sqrt{3}$ centred at the origin. We take a cube $Q$ inscribed in the sphere, and subdivide each face into a square grid with spacing $\varepsilon_{k}:=2 / k$. This induces a triangulation on $\partial Q$ by isosceles right triangles, whose restriction to each face satisfies $\left(\mathrm{H}_{4}\right)$. We pull back this triangulation to the sphere using the Lipschitz map $x \in \partial Q \mapsto x /|x|$. A similar construction can be carried out for any closed surface $M$, by noting that $M$ is bilipschitz equivalent to the boundary of a polyhedron whose faces are finite unions of squares. In the left Figure 2 we present another triangulation of the sphere that satisfy our set of hypothesis $\left(\mathrm{H}_{1}\right)-\left(\mathrm{H}_{4}\right)$. A triangulation that does not satisfies $\left(\mathrm{H}_{4}\right)$ is illustrated in the Figure 2 on the right.

The main characters of our analysis will be unit-norm tangent discrete vector fields on $M \cap \mathcal{T}_{\varepsilon}^{0}$, namely maps

$$
\mathbf{v}_{\varepsilon}: \mathcal{T}_{\varepsilon}^{0} \rightarrow \mathbb{R}^{3} \text { s.t. }|\mathbf{v}|=1 \text { and } \mathbf{v}_{\varepsilon}(i) \cdot \gamma(i)=0 \text { for any } i \in \mathcal{T}_{\varepsilon}^{0} .
$$

We will denote with $\mathrm{T}\left(\mathcal{T}_{\varepsilon} ; \mathbb{S}^{2}\right)$ the space of such discrete vector fields. Given $\mathbf{v}_{\varepsilon} \in \mathrm{T}\left(\mathcal{T}_{\varepsilon} ; \mathbb{S}^{2}\right), \widehat{\mathbf{v}}_{\varepsilon}: \widehat{M}_{\varepsilon} \rightarrow \mathbb{R}^{3}$ denotes the piecewise-affine interpolant of $\mathbf{v}_{\varepsilon}$. Note that $\widehat{\mathbf{v}}_{\varepsilon}$ can be represented using the basis functions $\widehat{\varphi}_{\varepsilon, i}$ in this way:

$$
\widehat{\mathbf{v}}_{\varepsilon}=\sum_{j \in \mathcal{T}_{\varepsilon}^{0}} \mathbf{v}_{\varepsilon}(j) \widehat{\varphi}_{\varepsilon, j}
$$



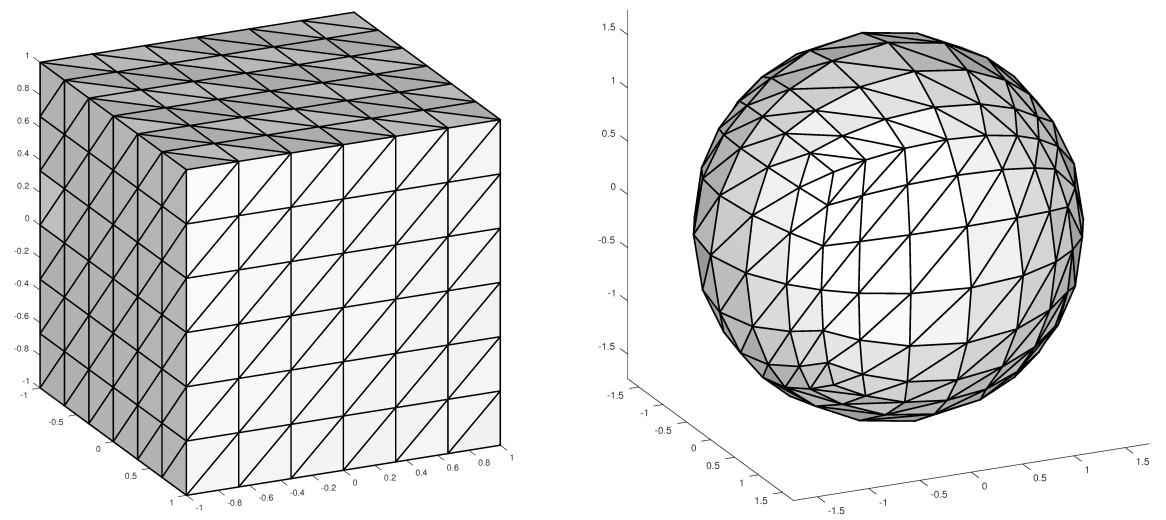

Fig. 1 Left: a uniform triangulation by isosceles right triangles on the boundary of a cube. Right: the same triangulation is mapped to a sphere, by renormalizing the coordinates of each vertex.
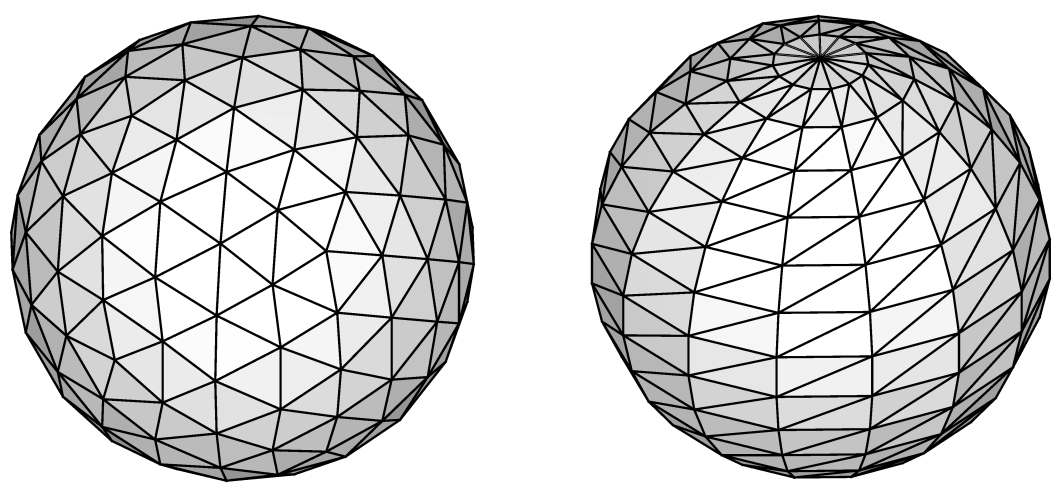

Fig. 2 Left: an icosphere. This triangulation is obtained by subdividing the faces of a regular icosahedron into smaller triangles, then projecting the vertices to the sphere. By refining this construction, one obtains a sequence of triangulations that satisfies $\left(\mathrm{H}_{1}\right)-\left(\mathrm{H}_{4}\right)$. Right: an $U V$-sphere, obtained by mapping a uniform grid on the square via spherical coordinates. The meshes produced by this method do not satisfy $\left(\mathrm{H}_{4}\right)$ nor $\left(\mathrm{H}_{2}\right)$, because the number of neighbours of the north and south poles blows up as the mesh is refined.

In a similar way, $\widehat{\gamma}_{\varepsilon}$ denotes the piecewise-affine interpolant of $\gamma$ restricted to $\mathcal{T}_{\varepsilon}^{0}$.

Remark 6 In computational applications, it might be convenient to define the set of discrete vector fields (11) using some numerical approximation $\gamma_{\varepsilon}$ of $\gamma$, instead of $\gamma$ itself. The arguments in this paper could easily be adapted to cover this case as well, provided that the approximation $\gamma_{\varepsilon}$ satisfies an a priori bound such as

$$
\sup _{i \in \mathcal{T}_{\varepsilon}^{0}}\left|\gamma_{\varepsilon}(i)-\gamma(i)\right| \leq C \varepsilon
$$




\subsection{The discrete energy}

Given a discrete field $\mathbf{v}_{\varepsilon} \in \mathrm{T}\left(\mathcal{T}_{\varepsilon}, \mathbb{S}^{2}\right)$, we consider the discrete XY-energy

$$
X Y_{\varepsilon}\left(\mathbf{v}_{\varepsilon}\right):=\frac{1}{2} \sum_{i \neq j \in \mathcal{T}_{\varepsilon}^{0}} \kappa_{\varepsilon}^{i j}\left|\mathbf{v}_{\varepsilon}(i)-\mathbf{v}_{\varepsilon}(j)\right|^{2},
$$

where the $\kappa_{\varepsilon}^{i j}$ 's are the coefficients of the stiffness matrix, defined in $\left(\mathrm{H}_{3}\right)$. Because the support of the hat function $\widehat{\varphi}_{\varepsilon, i}$ only intersects the triangles that are adjacent to $i$, we have $\kappa_{\varepsilon}^{i j}=0$ if the vertices $i, j$ are not adjacent. Hence, the XY-energy is indeed defined by a nearest-neighbours interaction. Moreover, due to (12) we have

$$
X Y_{\varepsilon}\left(\mathbf{v}_{\varepsilon}\right)=\frac{1}{2} \int_{\widehat{M}_{\varepsilon}}\left|\nabla_{\varepsilon} \widehat{\mathbf{v}}_{\varepsilon}\right|^{2} \mathrm{~d} S
$$

\subsection{The defect-free case}

Before addressing the problem of generation of defects, it is important to understand what happens for a shell $M$ with $\chi(M)=0$. In this case, the topology of $M$ does not force the formation of singularities as the PoincaréHopf Theorem admits configurations with $d_{i}=0$ for any $i$. Moreover, the results in [47] and [18] guarantee that the set

$$
\begin{gathered}
W_{\tan }^{1,2}\left(M ; \mathbb{S}^{2}\right):=\left\{\mathbf{v}: M \rightarrow \mathbb{R}^{3},|\mathbf{v}(x)|=1, \mathbf{v}(x) \in \mathrm{T}_{x} M \text { for a.a. } x \in M\right. \\
\left.|\mathrm{D} \mathbf{v}| \in L^{2}(M)\right\}
\end{gathered}
$$

is non empty. Consequently, for a compact surface without boundary and $\chi(M)=0$, the following energy $E_{\text {extr }}$ is well defined

$$
E_{\text {extr }}(\mathbf{v}):=\int_{M} e(\mathbf{v}) \mathrm{d} S, \quad e(\mathbf{v}):=|\mathrm{D} \mathbf{v}|^{2}+\frac{1}{2}|\mathrm{~d} \gamma[\mathbf{v}]|^{2} .
$$

When $\mathbf{v}$ is of unit norm, this energy governs the statics of a Nematic Shell (of genus 1 ) in the one constant approximation when one takes into account also extrinsic effects (see [36], [37] and [47]). Indeed, differently from (1), this energy includes the term with $\mathrm{d} \gamma$ that takes into account how the surface $M$ sits in the tridimensional space $\mathbb{R}^{3}$. We refer to the papers [36], [37] and [47] for a detailed analysis of $E_{\text {extr }}$ and for a discussions about the differences in selecting minimizers between (1) and (14). Interestingly, the energy (14) emerges as the discrete to continuum limit of the energies $X Y_{\varepsilon}$. In fact, suppose $M$ is a compact surface without boundary with $\chi(M)=0$. Let $\mathcal{T}_{\varepsilon}$ be a triangulation of $M$ satisfying the conditions $\left(\mathrm{H}_{1}\right),\left(\mathrm{H}_{2}\right)$ and $\left(\mathrm{H}_{3}\right)$. Now, given a smooth vector field $\mathbf{v}$ of unit norm we consider the discrete vector field given by the restriction of $\mathbf{v}$ to the nodes of the triangulation, namely $\mathbf{v}_{\varepsilon}(i):=\mathbf{v}(i)$ for $i \in \mathcal{T}_{0}^{\varepsilon}$. It is not difficult to realize that

$$
\lim _{\varepsilon \rightarrow 0} X Y_{\varepsilon}\left(\mathbf{v}_{\varepsilon}\right)=\lim _{\varepsilon \rightarrow 0} \frac{1}{2} \int_{\widehat{M}_{\varepsilon}}\left|\nabla_{\varepsilon} \widehat{\mathbf{v}}_{\varepsilon}\right|^{2} \mathrm{~d} S=\frac{1}{2} \int_{M}\left|\nabla_{\mathrm{s}} \mathbf{v}\right|^{2} \mathrm{~d} S=E_{\operatorname{extr}}(\mathbf{v}),
$$


where we used the fact that the directions tangent to $\widehat{M}_{\varepsilon}$ uniformly converge to directions that are tangent to $M$ together with (7). This discrete to continuum limit can be actually made rigorous in terms of $\Gamma$-convergence. More precisely, we have

Proposition 1. Suppose that the assumptions $\left(\mathrm{H}_{1}\right),\left(\mathrm{H}_{2}\right)$ and $\left(\mathrm{H}_{3}\right)$ are satisfied. Then, $X Y_{\varepsilon} \Gamma$-converges with respect to weak convergence of $L^{2}\left(M ; \mathbb{R}^{3}\right)$ to the functional

$$
E_{\operatorname{extr}}(\mathbf{v}):= \begin{cases}\frac{1}{2} \int_{M}|\mathrm{D} \mathbf{v}|^{2}+|\mathrm{d} \gamma[\mathbf{v}]|^{2} \mathrm{~d} S, & \text { if } \mathbf{v} \in W_{\tan }^{1,2}\left(M ; \mathbb{S}^{2}\right) \\ +\infty, & \text { otherwise in } L^{2}\left(M ; \mathbb{R}^{3}\right) .\end{cases}
$$

The proof of this Proposition follows routine arguments in the analysis of discrete to continuum limits. Therefore we skip it and we refer to [1], [16], [17] and [45, Theorem III.2].

\subsection{Configuration with defects: the zeroth order $\Gamma$-limit}

Towards the analysis of defects, we briefly introduce the important notion of discrete vorticity measure. This measure will be a kind of discrete notion of jacobian for the discrete vector field $\mathbf{v}_{\varepsilon}$ in $\mathrm{T}\left(\mathcal{T}_{\varepsilon} ; \mathbb{S}^{2}\right)$. As it happens for the discrete flat case and for Ginzburg Landau case, the vorticity measure of the sequence $\mathbf{v}_{\varepsilon}$ will provide all the informations regarding the emergence of the defects in the $\varepsilon \searrow 0$ limit. Even if we will precisely introduce this measure in the next Subsection 4.5, we briefly present it now for the sake of clarity. Given a triangle $T \in \mathcal{T}_{\varepsilon}$ we let $\left(i_{0}, i_{1}, i_{2}\right)$ be the vertices of $T$, sorted in counterclockwise order with respect to the orientation induced by $\gamma$, and let $i_{3}:=i_{0}$. For any triangle $T, \widehat{\mu}_{\varepsilon}\left(\mathbf{v}_{\varepsilon}\right)\llcorner T$ is supported on the barycenter of $T$ and

$$
\widehat{\mu}_{\varepsilon}\left(\mathbf{v}_{\varepsilon}\right)[T]:=\sum_{k=0}^{2}\left(\frac{\gamma\left(i_{k}\right)+\gamma\left(i_{k+1}\right)}{2}, \mathbf{v}_{\varepsilon}\left(i_{k}\right) \times \mathbf{v}_{\varepsilon}\left(i_{k+1}\right)\right) .
$$

Our notion of vorticity measure differs from other ones that appear in the mathematical literature on the XY-model (see e.g. [2]), in that it is not integervalued. However, it is possible to define a measure that approximates $\widehat{\mu}_{\varepsilon}\left(\mathbf{v}_{\varepsilon}\right)$, in a suitable sense, and takes integer values, thus being closer to the approach of [2] (see Section 4.5). In the limit $\varepsilon \rightarrow 0$, the appearance of defects is related to a measure concentrated on a finite number of points $\left\{x_{1}, \ldots, x_{k}\right\}$ in $M$. We will denote by $X$ the set of measures on $M$ of the form

$$
\mu=\sum_{i=1}^{k} d_{i} \delta_{x_{i}},
$$

where $k \in \mathbb{N}, d_{i} \in \mathbb{Z}$ are such that $\sum_{i} d_{i}=\chi(M)$ and $x_{i} \in M$ for $i \in$ $\{1, \ldots, k\}$. The space $X$ will be endowed with the topology of flat convergence, that is, the topology induced by the dual norm of Lipschitz functions.

Here is the precise statement of the first main result of the paper. 
Theorem A. Suppose that the assumptions $\left(\mathrm{H}_{1}\right),\left(\mathrm{H}_{2}\right)$ and $\left(\mathrm{H}_{3}\right)$ are satisfied. Then, the following results hold.

(i) Compactness. If $\left(\mathbf{v}_{\varepsilon}\right)$ is a sequence in $\mathrm{T}\left(\mathcal{T}_{\varepsilon} ; \mathbb{S}^{2}\right)$ that satisfies the energy bound

$$
X Y_{\varepsilon}\left(\mathbf{v}_{\varepsilon}\right) \leq \Lambda|\log \varepsilon|
$$

then, up to subsequences, $\hat{\mu}_{\varepsilon}\left(\mathbf{v}_{\varepsilon}\right) \stackrel{\text { flat }}{\longrightarrow} 2 \pi \mu-G \mathrm{~d} S$ for some $\mu \in X$.

(ii) $\Gamma$-liminf inequality. Let $\mathbf{v}_{\varepsilon} \in \mathrm{T}\left(\mathcal{T}_{\varepsilon} ; \mathbb{S}^{2}\right)$ be such that $\hat{\mu}_{\varepsilon}\left(\mathbf{v}_{\varepsilon}\right) \stackrel{\text { flat }}{\longrightarrow} 2 \pi \mu-$ $G \mathrm{~d} S$ for some $\mu \in X$. Then, there holds

$$
\liminf _{\varepsilon \rightarrow 0} \frac{X Y_{\varepsilon}\left(\mathbf{v}_{\varepsilon}\right)}{|\log \varepsilon|} \geq \pi|\mu|(M) .
$$

(iii) $\Gamma$-limsup inequality. For any measure $\mu \in X$ there exists a sequence $\left(\mathbf{v}_{\varepsilon}\right)$ in $\mathrm{T}\left(\mathcal{T}_{\varepsilon} ; \mathbb{S}^{2}\right)$ such that $\hat{\mu}_{\varepsilon}\left(\mathbf{v}_{\varepsilon}\right) \stackrel{\text { flat }}{\longrightarrow} 2 \pi \mu-G \mathrm{~d} S$ and

$$
\limsup _{\varepsilon \rightarrow 0} \frac{X Y_{\varepsilon}\left(\mathbf{v}_{\varepsilon}\right)}{|\log \varepsilon|} \leq \pi|\mu|(M) .
$$

This Theorem will be proved in the next Section 5 (see Proposition 4). In this Proposition we will actually prove a slightly stronger result, where the $\Gamma$-liminf inequality (ii) is replaced by (a local version of)

$$
X Y_{\varepsilon}\left(\mathbf{v}_{\varepsilon}\right) \geq \pi|\mu|(M)|\log \varepsilon|-C .
$$

3.5 Location and energetics of defects: the Renormalized Energy and the core energy

Beside discussing the generation of defects, we are interested in understanding the energetics of defects configurations and, consequently, locate the defects on the surface $M$. This program is achieved by the analysis of the so called Renormalized Energy $\mathbb{W}$ introduced by Brezis, Bethuel and Hélein for the Ginzburg Landau equation in [12]. In this paper, we obtain the Rernomalized Energy as the (first order) $\Gamma$-limit of the discrete energy $X Y_{\varepsilon}$ as in [41], [3], [2] for the euclidean case.

The Renormalized Energy emerges as the $\Gamma$-limit with respect to the strong $L^{2}$ convergence of the following rescaled energy

$$
X Y_{\varepsilon}\left(\mathbf{v}_{\varepsilon}\right)-\pi \mathscr{K}|\log \varepsilon|,
$$

where $\mathscr{K}$ is a positive, even integer such that $|\chi(M)| \leq \mathscr{K}$. Now, we introduce the concept of Renormalized Energy. Following [2], we introduce the following class of vector fields in $M: \mathcal{V}_{\mathscr{K}}$ is the set of measurable fields v: $M \rightarrow \mathbb{S}^{2}$ such that there exist $\left(x_{i}\right)_{i=1}^{\mathscr{K}} \in M^{\mathscr{K}}$ such that

$$
\mathbf{v} \in W_{\text {tan,loc }}^{1,2}\left(M \backslash \bigcup_{i=1}^{\mathscr{K}} x_{i} ; \mathbb{S}^{2}\right), \quad\left|\operatorname{ind}\left(\mathbf{v}, x_{i}\right)\right|=1 \text { for any } i=1, \ldots, \mathscr{K} .
$$


We define the intrinsic Renormalized Energy as (see [2, Eq. (4.22)]):

$$
\mathbb{W}_{\text {intr }}(\mathbf{v}):=\lim _{\delta \rightarrow 0}\left(\frac{1}{2} \int_{M_{\delta}}|\mathrm{D} \mathbf{v}|^{2} \mathrm{~d} S-\mathscr{K} \pi|\log \delta|\right) \quad \text { for } \mathbf{v} \in \mathcal{V}_{\mathscr{K}}
$$

where, given $\mathbf{v} \in \mathcal{V}_{\mathscr{K}}$ and $\delta>0$ so small that the balls $B_{\delta}\left(x_{i}\right)$ are pairwise disjoint, we have set $M_{\delta}:=M \backslash \bigcup_{i=1}^{\mathscr{K}} B_{\delta}\left(x_{i}\right)$. As we will see in Subsection 6.1, the functional $\mathbb{W}_{\text {intr }}$ is well defined.

Now, assume that $\mathbf{v} \in \mathcal{V}_{\mathscr{K}}$. Since in particular, $|\mathbf{v}|=1$, we have

$$
|\mathrm{d} \gamma[\mathbf{v}]| \leq C \quad \text { a.e. in } M
$$

where the constant $C$ depends only on $M$. Thus, the following quantity exists in $[-\infty,+\infty]$ :

$$
\mathbb{W}(\mathbf{v}):=\mathbb{W}_{\text {intr }}(\mathbf{v})+\frac{1}{2} \int_{M}|\mathrm{~d} \gamma[\mathbf{v}]|^{2} \mathrm{~d} S
$$

$\mathbb{W}$ will be called the Renormalized Energy. Note that $\mathbb{W}$ contains both an intrinsic and an extrinsic term but, due to (19), the latter is always finite. This shows, as expected, that the concentration of the energy is due to the Dirichlet part of $E_{\text {extr }}$ in (14).

For any $\mathbf{v} \in \mathcal{V}_{\mathscr{K}}$ such that $\mathbf{v} \in W_{\text {loc }}^{1,2}\left(M \backslash \cup_{i} x_{i}\right)$, we consider the measure $\sum_{i} \operatorname{ind}\left(\mathbf{v}, x_{i}\right) \delta_{x_{i}}-G \mathrm{~d} S$, denoted (with a slight abuse of notation) $\star \mathrm{d} \jmath(\mathbf{v})$. If $\mathbb{W}(\mathbf{v})<+\infty$, it turns out that $\star \mathrm{d} \jmath(\mathbf{v})$ coincides with the Jacobian determinant of $\mathbf{v}$, in the sense of distributions (see Lemmas 6 and 15).

Remark 7 The Renormalized Energy on simply-connected planar domains, as defined by Bethuel, Brezis and Hélein in [12], is a function of the locations of the defects, $x=\left(x_{i}\right)_{i=1}^{\mathscr{K}}$, and their charge $d=\left(d_{i}\right)_{i=1}^{\mathscr{K}}$. In the curved setting, the Renormalized Energy also depends on the so-called flux integrals of $\mathbf{v}$, $\left(\Phi_{k}\right)_{k=1}^{2 \mathfrak{g}} \in(\mathbb{R} / 2 \pi \mathbb{Z})^{2 \mathfrak{g}}$, where $\mathfrak{g}=1-\chi(M) / 2$ is the genus of $M$ (see $[25$, Section 2]). These quantities need to be introduced, to compensate for the lack of simple connectedness of $M$. The relation between the Renormalized Energy $W$ as defined in [25] and ours is the following (compare with [2, Eq. (4.23)] for the planar case):

$$
\inf _{\Phi \in \mathcal{L}(x, d)} W(x, d, \Phi)=\inf \left\{\mathbb{W}_{\operatorname{intr}}(\mathbf{v}): \mathbf{v} \in \mathcal{V}_{\mathscr{K}}, \star \mathrm{d} \jmath(\mathbf{v})=2 \pi \sum_{i=1}^{\mathscr{K}} d_{i} \delta_{x_{i}}-G\right\}
$$

where $\mathcal{L}(x, d)$ denotes the set of admissible fluxes, given $(x, d)$. The proof of this property is analogous to the proof of the corresponding relation in Euclidean setting, therefore we skip it. Interestingly, the quantity $W$ can be expressed in terms of the Green function for the Laplace-Beltrami operator of $M$; see [25, Proposition 2]. 
If we assume that also Hypothesis $\left(\mathrm{H}_{4}\right)$ holds, for each singularity $x_{i}$ we can construct the so-called core energy, a positive number that takes into account the energy in the core of the defect at $x_{i}$. For the sake of clarity, we briefly introduce it here and we refer to Section 6.2 (in particular Proposition 5, for the definition) for the details. We fix a point $\bar{x} \in M$ and a positive $\delta$ smaller than the injectivity radius of $M$. Then, for $\varepsilon>0, \delta>0$, we set

$$
\gamma_{\bar{x}}(\varepsilon, \delta):=\min _{\mathbf{v} \in \mathrm{T}\left(\mathcal{T}_{\varepsilon}, S^{2}\right)}\left\{\frac{1}{2} \int_{\left.\widehat{B_{\delta}(\bar{x})}\right)_{\varepsilon}}\left|\nabla_{\varepsilon} \widehat{\mathbf{v}}\right|^{2} \mathrm{~d} S: \quad \mathbf{v}=\mathbf{g} \text { on } \partial_{\varepsilon} B_{\delta}(\bar{x})\right\} .
$$

The boundary condition $\mathbf{g}$ is a minimizer with index one of the energy (14) in the anulus of radii $\delta / 2$ and $\delta$ centered in $\bar{x}$. In the euclidean case (see [2]), $\mathbf{g}$ is, up to rotations, an hedge-hog. In the formula ahead,

$$
\widehat{B_{\delta}(\bar{x})} \varepsilon:=\bigcup_{P(T) \subseteq B_{\delta}(\bar{x})} T,
$$

$\partial_{\varepsilon} B_{\delta}(\bar{x}):=\partial\left(\widehat{B_{\delta}(\bar{x})_{\varepsilon}}\right) \cap \mathcal{T}_{\varepsilon}^{0}$, and $\widehat{\mathbf{v}}$ is the affine interpolant of the discrete field $\mathbf{v}$.

The core energy emerges in the limits $\varepsilon \rightarrow 0$ and $\delta \rightarrow 0$ (exactly in this order) in (22). More precisely, if the sequence of triangulations $\left(\mathcal{T}_{\varepsilon}\right)$ satisfies $\left(\mathrm{H}_{1}\right),\left(\mathrm{H}_{2}\right),\left(\mathrm{H}_{3}\right)$ and $\left(\mathrm{H}_{4}\right)$, for any $\bar{x} \in M$ the following limits are finite and coincide (see Proposition 5):

$$
\gamma(\bar{x}):=\lim _{\delta \rightarrow 0} \liminf _{\varepsilon \rightarrow 0}\left(\gamma(\varepsilon, \delta)-\pi \log \frac{\delta}{\varepsilon}\right)=\lim _{\delta \rightarrow 0} \limsup _{\varepsilon \rightarrow 0}\left(\gamma(\varepsilon, \delta)-\pi \log \frac{\delta}{\varepsilon}\right) .
$$

The core energy is defined as the value of this limit. As the proof of Proposition 5 will show, the core energy $\gamma(\bar{x})$ depends on the structure of the triangulation around the point $\bar{x}$. This new feature is a discrete phenomenon and can be (at least heuristically) explained by the following analogy. In their recent papers [25] and [26], Jerrard and Ignat show that the core energy that emerges in the $\Gamma$-convergence of the Ginzburg Landau energy for tangent vector fields only depends (as in the euclidean case) on the confining potential in the energy. In Ginzburg Landau theory the topological constraints of the problem are relaxed by relaxing, via the confining potential, the unit norm constraint. In the discrete setting, the relaxation of the topological constraints comes by introducing a triangulation of $M$ and by depositing a discrete vector field at the nodes of the triangulation. This analogy hence suggests that the dependence of the core energy on the triangulation is, somehow, natural. On the other hand, the proof of Proposition 5 - which proceeds by reducing to the case the problem is defined on $\mathbb{R}^{2}$ — suggests that $\gamma(\bar{x})$ should not depend on geometric properties of the surface.

It is interesting to observe that if one considers, in the euclidean case, an $X Y$ type energy on a square lattice or on triangular lattice (even with some anisotropy — see, e.g., [2] and [22]) then the core energy turns out to be a positive constant that depends only on the details of the energy since the local 
structure of the mesh is essentially the same around every point. It would be intriguing to study the dependence of the core energy on the point even in the euclidean case for less structured discretizations.

To correctly state our result, we finally need a proper continuous interpolation of the discrete vector field $\mathbf{v}_{\varepsilon}$. Thus, we define (see (36)) $\mathbf{w}_{\varepsilon}: M \rightarrow \mathbb{R}^{3}$ as

$$
\mathbf{w}_{\varepsilon}:=\widehat{\mathbf{v}}_{\varepsilon} \circ \widehat{P}_{\varepsilon}^{-1},
$$

where $\widehat{\mathbf{v}}_{\varepsilon}: \widehat{M}_{\varepsilon} \rightarrow \mathbb{R}^{3}$ is the affine interpolant of $\mathbf{v}_{\varepsilon}$ and $\widehat{P}_{\varepsilon}^{-1}$ is the inverse of the nearest-point projection, see $\left(\mathrm{H}_{1}\right)$.

The second main result is thus the following

Theorem B. Suppose that the assumptions $\left(\mathrm{H}_{1}\right),\left(\mathrm{H}_{2}\right),\left(\mathrm{H}_{3}\right)$ and $\left(\mathrm{H}_{4}\right)$ are satisfied. Then the following $\Gamma$-convergence result holds.

(i) Compactness. Let $\mathscr{K} \in \mathbb{N}$ and let $\mathbf{v}_{\varepsilon}$ be a sequence in $\mathrm{T}\left(\mathcal{T}_{\varepsilon} ; \mathbb{S}^{2}\right)$ for which there exists a positive constant $C_{\mathscr{K}}$ such that

$$
X Y_{\varepsilon}\left(\mathbf{v}_{\varepsilon}\right)-\mathscr{K} \pi|\log \varepsilon| \leq C_{\mathscr{K}} .
$$

Then, up to a subsequence, there holds

$$
\hat{\mu}_{\varepsilon}\left(\mathbf{v}_{\varepsilon}\right) \stackrel{\text { flat }}{\longrightarrow} 2 \pi \mu-G \mathrm{~d} S
$$

for some $\mu \in X$ with $\sum_{i=1}^{k}\left|d_{i}\right| \leq \mathscr{K}$. If $|\mu|=\mathscr{K}$, then $k=\mathscr{K} \equiv \chi(M)$ $\bmod 2,\left|d_{i}\right|=1$ for any $i$. Moreover, there exists $\mathbf{v} \in \mathcal{V}_{\mathscr{K}}$ with $\star \mathrm{d} \jmath(\mathbf{v})=$ $2 \pi \mu-G \mathrm{~d} S$ and a subsequence such that

$$
\mathbf{w}_{\varepsilon} \rightarrow \mathbf{v} \text { strongly in } L^{2}\left(M ; \mathbb{R}^{3}\right) \text { and weakly in } W_{\mathrm{loc}}^{1,2}\left(M \backslash \bigcup_{i=1}^{\mathscr{K}} x_{i} ; \mathbb{R}^{3}\right) \text {. }
$$

(ii) $\Gamma$-lim inf inequality. Let $\mathbf{v}_{\varepsilon} \in \mathrm{T}\left(\mathcal{T}_{\varepsilon} ; \mathbb{S}^{2}\right)$ be a sequence satisfying (23) with $\mathscr{K} \equiv \chi(M) \bmod 2$, and let $\mathbf{v} \in \mathcal{V}_{\mathscr{K}}$ such that $\hat{\mu}_{\varepsilon}\left(\mathbf{v}_{\varepsilon}\right) \stackrel{\text { flat }}{\longrightarrow} \star \mathrm{d} \jmath(\mathbf{v}), \mathbf{w}_{\varepsilon} \rightarrow \mathbf{v}$ as in (25). Then, there holds

$$
\liminf _{\varepsilon \rightarrow 0}\left(X Y_{\varepsilon}\left(\mathbf{v}_{\varepsilon}\right)-\mathscr{K} \pi|\log \varepsilon|\right) \geq \mathbb{W}(\mathbf{v})+\sum_{i=1}^{\mathscr{K}} \gamma\left(x_{i}\right),
$$

where $\gamma\left(x_{i}\right)$ is the core energy around each defect $x_{i}$ (see Proposition 5).

(iii) $\Gamma$-limsup inequality. Given $\mathbf{v} \in \mathcal{V}_{\mathscr{K}}$, there exists $\mathbf{v}_{\varepsilon} \in \mathrm{T}\left(\mathcal{T}_{\varepsilon} ; \mathbb{S}^{2}\right)$ such that $\hat{\mu}_{\varepsilon}\left(\mathbf{v}_{\varepsilon}\right) \stackrel{\text { flat }}{\longrightarrow} \star \mathrm{d} \jmath(\mathbf{v}), \mathbf{w}_{\varepsilon} \rightarrow \mathbf{v}$ as in (25) and

$$
\lim _{\varepsilon \rightarrow 0}\left(X Y_{\varepsilon}\left(\mathbf{v}_{\varepsilon}\right)-\mathscr{K} \pi|\log \varepsilon|\right)=\mathbb{W}(\mathbf{v})+\sum_{i=1}^{\mathscr{K}} \gamma\left(x_{i}\right) .
$$


As anticipated in the Introduction of the paper, the above Theorem entails a precise convergence result for the minima of $X Y_{\varepsilon}$. More precisely, the Fundamental Theorem of $\Gamma$-convergence gives that if we set $\mathbf{v}_{\varepsilon}^{*} \in \operatorname{argmin}_{\mathrm{T}\left(\mathcal{T}_{\varepsilon} ; \mathbb{S}^{2}\right)} X Y_{\varepsilon}$ and $\mathbf{w}_{\varepsilon}^{*}$ as in (36), there exist points $x_{1}, \ldots, x_{|\chi(M)|}$ and a unit-valued tangent field $\mathbf{v}^{*}$ such that

$$
\mathbf{w}_{\varepsilon}^{*} \rightarrow \mathbf{v}^{*} \quad \text { weakly in } W_{\mathrm{loc}}^{1,2}\left(M \backslash \bigcup_{i=1}^{|\chi(M)|} x_{i} ; \mathbb{R}^{3}\right) .
$$

Moreover, there holds

$$
\min _{\mathrm{T}\left(\mathcal{T}_{\varepsilon} ; \mathbb{S}^{2}\right)} X Y_{\varepsilon}=\pi|\chi(M)||\log \varepsilon|+\mathbb{W}\left(\mathbf{v}^{*}\right)+\sum_{i=1}^{|\chi(M)|} \gamma\left(x_{i}\right)+\mathrm{o}_{\varepsilon \rightarrow 0}(1) .
$$

Note that the fact that $\mathscr{K}=|\chi(M)|$ implies that there are only two circumstances for the charge of the defects: either $d_{i}=1$ for any $i=1 \ldots, \mathscr{K}$ either $d_{i}=-1$ for any $i=1, \ldots, \mathscr{K}$.

\section{Preliminaries}

4.1 Distance between triangulations

Let $\mathcal{S}, \mathcal{T}$ be two (finite) triangulations on $\mathbb{R}^{2}$. We assume that $\mathcal{S}, \mathcal{T}$ are quasiuniform of size $\mathrm{O}(1)$, i.e., there exists a constant $\Lambda$ such that, for any $T \in \mathcal{S} \cup \mathcal{T}$, the affine bijection $\phi_{T}$ from the reference triangle $T_{\text {ref }}$ to $T$ satisfies

$$
\max \left\{\operatorname{Lip}\left(\phi_{T}\right), \operatorname{Lip}\left(\phi_{T}^{-1}\right)\right\} \leq \Lambda
$$

(compare with $\left(\mathrm{H}_{2}\right)$ ). We define an isomorphism from $\mathcal{S}$ to $\mathcal{T}$ as a transformation

$$
\phi: \bigcup_{T \in \mathcal{S}} T \rightarrow \bigcup_{T \in \mathcal{T}} T
$$

that satisfies the following conditions:

(i) for any $T \in \mathcal{S}$, restricts to an affine map $T \rightarrow \mathbb{R}^{2}$;

(ii) $\phi$ restricts to a bijection $\mathcal{S}^{0} \rightarrow \mathcal{T}^{0}$;

(iii) any three vertices $i, j, k \in \mathcal{S}^{0}$ span a triangle in $\mathcal{S}$ if and only if $\phi(i), \phi(j)$, $\phi(k)$ span a triangle in $\mathcal{T}$.

We denote by $\operatorname{Iso}(\mathcal{S}, \mathcal{T})$ the set of isomorphisms from $\mathcal{S}$ to $\mathcal{T}$. Note that $\operatorname{Iso}(\mathcal{S}, \mathcal{T}) \subseteq C \cap W^{1,2}$. We also define

$$
d(\mathcal{S}, \mathcal{T}):=\inf _{\phi \in \operatorname{Iso}(\mathcal{S}, \mathcal{T})} \max _{i \in \mathcal{S}^{0}}|i-\phi(i)|
$$

(the infimum being equal to $+\infty$ if $\operatorname{Iso}(\mathcal{S}, \mathcal{T})=\emptyset$ ). The function $d$ defines a metric on the triangulations of the plane. 
Lemma 1 Let $\mathcal{S}, \mathcal{T}$ be two triangulations such that $\operatorname{Iso}(\mathcal{S}, \mathcal{T}) \neq \emptyset$. Suppose (30) is satisfied for some $\Lambda>0$. Then, there exists $\phi \in \operatorname{Iso}(\mathcal{S}, \mathcal{T})$ such that

$$
\max \left\{\operatorname{Lip}(\phi), \operatorname{Lip}\left(\phi^{-1}\right)\right\} \leq 1+C d(\mathcal{S}, \mathcal{T}),
$$

where $C$ is a positive constant that depends only on $\Lambda$.

Proof. Let $\phi \in \operatorname{Iso}(\mathcal{S}, \mathcal{T})$ be such that $|i-\phi(i)| \leq 2 d(\mathcal{S}, \mathcal{T})$ for any $i \in \mathcal{S}^{0}$. Since the restriction of $\phi$ to any triangle of $\mathcal{S}$ is affine, we deduce that

$$
\|\operatorname{Id}-\phi\|_{L^{\infty}(T)} \leq 2 d(\mathcal{S}, \mathcal{T})
$$

on each $T \in \mathcal{S}$. Using the assumption (30), and up to composition with an affine bijection, we can assume without loss of generality that $T$ is the triangle of vertices $(0,0),(1,0),(0,1)$. Since the space of affine functions on $T$ is finite-dimensional, the $L^{\infty}$ - and the $W^{1, \infty}$-norm of an affine map on $T$ are equivalent. Thus, $\|\mathrm{Id}-\phi\|_{W^{1, \infty}(T)} \leq C d(\mathcal{S}, \mathcal{T})$ and the lemma follows.

\subsection{The metric distorsion tensor}

By the assumption $\left(\mathrm{H}_{1}\right)$, the restriction of the nearest-point projection $\widehat{P}_{\varepsilon}$ from $\widehat{M}_{\varepsilon}$ to $M$ has a Lipschitz inverse $\widehat{P}_{\varepsilon}^{-1}: M \rightarrow \widehat{M}_{\varepsilon}$. Following [24], we use this pair of maps to compare $M$ with its polyhedral approximation $\widehat{M}_{\varepsilon}$. For any $x \in M$ such that $\widehat{P}_{\varepsilon}^{-1}(x)$ falls in the interior of a triangle of $\widehat{M}_{\varepsilon}$ (so that $\widehat{P}_{\varepsilon}^{-1}$ is smooth in a neighbourhood of $\left.x\right)$, we let $\mathbf{A}_{\varepsilon}(x)$ be the unique linear operator $\mathrm{T}_{x} M \rightarrow \mathrm{T}_{x} M$ that satisfies

$$
\left(\mathbf{A}_{\varepsilon}(x) \mathbf{X}, \mathbf{Y}\right)=\left(\mathrm{d} \widehat{P}_{\varepsilon}^{-1}(x)[\mathbf{X}], \mathrm{d} \widehat{P}_{\varepsilon}^{-1}(x)[\mathbf{Y}]\right)
$$

for any $\mathbf{X}, \mathbf{Y} \in \mathrm{T}_{x} M$. This defines (almost everywhere) a (1, 1)-tensor field $\mathbf{A}_{\varepsilon} \in L^{\infty}\left(M ; \mathrm{T} M \otimes \mathrm{T}^{*} M\right)$, which is called metric distorsion tensor in the terminology of [24]. The metric distorsion tensor is symmetric and positive definite, since the right-hand side of $(32)$ is. We introduce a norm $\|\cdot\|_{L^{\infty}(M)}$ on $L^{\infty}\left(M ; \mathrm{T} M \otimes \mathrm{T}^{*} M\right)$ by

$$
\|\mathbf{A}\|_{L^{\infty}(M)}:=\operatorname{essip}_{x \in M}\|\mathbf{A}(x)\|_{\mathrm{T} M \otimes \mathrm{T}^{*} M}
$$

where $\|\cdot\|_{\mathrm{T} M \otimes \mathrm{T}^{*} M}$ is the operator norm.

Lemma 2 Suppose that $\left(\mathcal{T}_{\varepsilon}\right)$ satisfies $\left(\mathrm{H}_{1}\right)$ and $\left(\mathrm{H}_{2}\right)$. Then, there holds

$$
\left\|\mathbf{A}_{\varepsilon}-\operatorname{Id}\right\|_{L^{\infty}(M)}+\left\|\mathbf{A}_{\varepsilon}^{-1}-\operatorname{Id}\right\|_{L^{\infty}(M)} \leq C \varepsilon .
$$

Proof. Let $\widehat{\boldsymbol{\nu}}_{\varepsilon}: \widehat{M}_{\varepsilon} \rightarrow \mathbb{R}^{3}$ be a unit normal field to $\widehat{M}_{\varepsilon}$, which is well defined (and constant) in the interior of each triangle. The assumption $\left(\mathrm{H}_{2}\right)$ implies that

$$
\left\|\widehat{\boldsymbol{\nu}}_{\varepsilon} \circ \widehat{P}_{\varepsilon}^{-1}-\gamma\right\|_{L^{\infty}(M)} \leq C \varepsilon, \quad\left\|\operatorname{dist}\left(\cdot, \widehat{M}_{\varepsilon}\right)\right\|_{L^{\infty}(M)} \leq C \varepsilon
$$


for some $\varepsilon$-independent constant $C$. (One can write $M$ as a smooth graph locally around a point $x \in M$, then use a Taylor expansion; the constant $C$ can be chosen uniformly with respect to $x$, by a compactness argument.) Thanks to $\left[24\right.$, Theorem 1], which gives a formula for $\mathbf{A}_{\varepsilon}$ in terms of $\left(\widehat{\boldsymbol{\nu}}_{\varepsilon} \circ \widehat{P}_{\varepsilon}^{-1}, \gamma\right)$ and $\operatorname{dist}\left(\cdot, \widehat{M}_{\varepsilon}\right)$, we deduce

$$
\left\|\mathbf{A}_{\varepsilon}-\operatorname{Id}\right\|_{L^{\infty}(M)} \leq C \varepsilon
$$

Now, the definition (32) of $\mathbf{A}_{\varepsilon}$, together with the fact that $\widehat{P}_{\varepsilon}^{-1}$ has a Lipschitz inverse $\widehat{P}_{\varepsilon}$ and $\operatorname{Lip}\left(\widehat{P}_{\varepsilon}\right) \leq \Lambda$ by $\left(\mathrm{H}_{1}\right)$, imply that

$$
\left|\mathbf{A}_{\varepsilon}(x) \mathbf{X}\right| \geq C|\mathbf{X}|
$$

for some constant $C$ depending on $\Lambda$, a.e. $x \in M$ and all $\mathbf{X} \in \mathrm{T}_{x} M$, whence $\left\|\mathbf{A}_{\varepsilon}^{-1}\right\|_{L^{\infty}(M)} \leq C$. Thus, we have

$$
\left\|\mathbf{A}_{\varepsilon}^{-1}-\operatorname{Id}\right\|_{L^{\infty}(M)} \leq\left\|\mathbf{A}^{-1}\right\|_{L^{\infty}(M)}\left\|\operatorname{Id}-\mathbf{A}_{\varepsilon}\right\|_{L^{\infty}(M)} \stackrel{(33)}{\leq} C \varepsilon .
$$

Let $g_{\varepsilon} \in L^{\infty}\left(M ; \mathrm{T}^{*} M^{\otimes 2}\right)$ be the metric on $M$ defined by $g_{\varepsilon}(\mathbf{X}, \mathbf{Y}):=$ $\left(\mathbf{A}_{\varepsilon} \mathbf{X}, \mathbf{Y}\right)$, for any smooth fields $\mathbf{X}$ and $\mathbf{Y}$ on $M$. Given a function $u \in$ $W^{1,2}(M)$, one can define the Sobolev $W^{1,2}$-seminorm of $u$ with respect to $g_{\varepsilon}$, i.e.

$$
|u|_{W_{\varepsilon}^{1,2}(M)}^{2}:=\int_{M}\left(\mathbf{A}_{\varepsilon}^{-1} \nabla_{\mathrm{s}} u, \nabla_{\mathrm{s}} u\right)\left(\operatorname{det} \mathbf{A}_{\varepsilon}\right)^{1 / 2} \mathrm{~d} S .
$$

By construction (32), the map $\widehat{P}_{\varepsilon}^{-1}$ is an isometry between $M$, equipped with the metric $g_{\varepsilon}$, and $\widehat{M}_{\varepsilon}$, with the metric induced by $\mathbb{R}^{3}$. Therefore, given $v \in$ $W^{1,2}\left(\widehat{M}_{\varepsilon} ; \mathbb{R}\right)$ and a Borel set $U \subseteq M$, there holds

$$
\left|v \circ \widehat{P}_{\varepsilon}^{-1}\right|_{W_{\varepsilon}^{1,2}(U)}^{2}=\int_{\widehat{P}_{\varepsilon}^{-1}(U)}\left|\nabla_{\varepsilon} v\right|^{2} \mathrm{~d} S .
$$

Arguing component-wise, we see that the same equality holds for a (not necessarily tangent) vector field $\mathbf{v}: \widehat{M}_{\varepsilon} \rightarrow \mathbb{R}^{3}$ in place of $v$.

\subsection{Interpolants of discrete fields}

Using assumption $\left(\mathrm{H}_{1}\right)$, to any discrete vector field $\mathbf{v}_{\varepsilon} \in \mathrm{T}\left(\mathcal{T}_{\varepsilon} ; \mathbb{S}^{2}\right)$ (see (11)) we can associate a continuous field $\mathbf{w}_{\varepsilon}: M \rightarrow \mathbb{R}^{3}$ by setting

$$
\mathbf{w}_{\varepsilon}:=\widehat{\mathbf{v}}_{\varepsilon} \circ \widehat{P}_{\varepsilon}^{-1},
$$

where $\widehat{\mathbf{v}}_{\varepsilon}: \widehat{M}_{\varepsilon} \rightarrow \mathbb{R}^{3}$ is the affine interpolant of $\mathbf{v}_{\varepsilon}$. The field $\mathbf{w}_{\varepsilon}$ is Lipschitzcontinuous and satisfies $\mathbf{w}_{\varepsilon}=\mathbf{v}_{\varepsilon}$ on $\mathcal{T}_{\varepsilon}^{0}$, but it is not tangent to $M$ nor unit-valued, in general. However, one can still prove some useful properties. 
Lemma 3 Suppose that $\left(\mathrm{H}_{1}\right),\left(\mathrm{H}_{2}\right),\left(\mathrm{H}_{3}\right)$ are satisfied. Then, for any $\varepsilon \in$ $\left(0, \varepsilon_{0}\right]$ and any discrete field $\mathbf{v}_{\varepsilon} \in \mathrm{T}\left(\mathcal{T}_{\varepsilon} ; \mathbb{S}^{2}\right), \mathbf{w}_{\varepsilon}$ is Lipschitz-continuous with Lipschitz constant

$$
\operatorname{Lip}\left(\mathbf{w}_{\varepsilon}\right) \leq C \varepsilon^{-1}
$$

and, for any subset $\widehat{U} \subseteq \widehat{M}_{\varepsilon}$ that can be written as union of triangles of $\mathcal{T}_{\varepsilon}$, there holds

$$
X Y_{\varepsilon}\left(\mathbf{v}_{\varepsilon}, \widehat{U}\right):=\frac{1}{2} \sum_{i, j \in \mathcal{T}_{\varepsilon}^{0} \cap \widehat{U}} \kappa_{\varepsilon}^{i j}\left|\mathbf{v}_{\varepsilon}(i)-\mathbf{v}_{\varepsilon}(j)\right|^{2}=\frac{1}{2}\left|\mathbf{w}_{\varepsilon}\right|_{W_{\varepsilon}^{1,2}(P(\widehat{U}))}^{2}
$$

Proof. From the very definition of $\mathbf{w}_{\varepsilon}:=\widehat{\mathbf{v}}_{\varepsilon} \circ \widehat{P}_{\varepsilon}^{-1}$, it follows that

$$
\operatorname{Lip}\left(\mathbf{w}_{\varepsilon}\right) \stackrel{\left(\mathrm{H}_{1}\right)}{\leq} C \operatorname{Lip}\left(\widehat{\mathbf{v}}_{\varepsilon}\right) \leq C \sup _{[i, j] \in \mathcal{T}_{\varepsilon}^{1}} \frac{\left|\mathbf{v}_{\varepsilon}(i)-\mathbf{v}_{\varepsilon}(j)\right|}{|i-j|} \stackrel{\left(\mathrm{H}_{2}\right)}{\leq} C \varepsilon^{-1}
$$

To prove (38), it is enough to combine (10) with (35).

Lemma 4 Suppose that $\left(\mathcal{T}_{\varepsilon}\right)$ satisfies $\left(\mathrm{H}_{1}\right),\left(\mathrm{H}_{2}\right)$. Then, there exists a contant $C$ such that, for any $\varepsilon \in\left(0, \varepsilon_{0}\right]$ and any $\mathbf{v}_{\varepsilon} \in \mathrm{T}\left(\mathcal{T}_{\varepsilon} ; \mathbb{S}^{2}\right)$, there holds

$$
\left\|\left(\mathbf{w}_{\varepsilon}, \gamma\right)\right\|_{L^{\infty}(M)} \leq C \varepsilon
$$

Proof. Every point $x \in \widehat{M}_{\varepsilon}$ can be written in the form $x=\lambda_{0} i_{0}+\lambda_{1} i_{1}+\lambda_{2} i_{2}$, where $i_{k} \in \mathcal{T}_{\varepsilon}^{0}$ and $\lambda_{k} \geq 0, \lambda_{0}+\lambda_{1}+\lambda_{2}=1$. Using the definition of the affine interpolant, and the fact that $\left(\mathbf{v}_{\varepsilon}\left(i_{k}\right), \gamma\left(i_{k}\right)\right)=0$, we can write

$$
\begin{aligned}
\left|\left(\widehat{\mathbf{v}}_{\varepsilon}(x),\left(\gamma \circ \widehat{P}_{\varepsilon}\right)(x)\right)\right| & \leq \sum_{k=0}^{2} \lambda_{k}\left|\left(\mathbf{v}_{\varepsilon}\left(i_{k}\right),\left(\gamma \circ \widehat{P}_{\varepsilon}\right)(x)-\left(\gamma \circ \widehat{P}_{\varepsilon}\right)\left(i_{k}\right)\right)\right| \\
& \leq\|\nabla(\gamma \circ P)\|_{L^{\infty}(U)} \sup _{T \in \mathcal{T}_{\varepsilon}} \operatorname{diam}(T) .
\end{aligned}
$$

Thus, using the smoothness of $\gamma$ and the assumptions $\left(\mathrm{H}_{1}\right),\left(\mathrm{H}_{2}\right)$, we deduce

$$
\left\|\left(\mathbf{w}_{\varepsilon}, \gamma\right)\right\|_{L^{\infty}(M)}=\left\|\left(\widehat{\mathbf{v}}_{\varepsilon}, \gamma \circ \widehat{P}_{\varepsilon}\right)\right\|_{L^{\infty}\left(\widehat{M}_{\varepsilon}\right)} \leq C \varepsilon
$$

The following property is well-known in the flat case (see e.g. [1, Lemma 2]).

Lemma 5 Suppose that $\left(\mathrm{H}_{2}\right)$ is satisfied. Then, there exists a positive constant $C$ such that, for any $0<\varepsilon \leq \varepsilon_{0}$ and any discrete field $\mathbf{v}_{\varepsilon} \in \mathrm{T}\left(\mathcal{T}_{\varepsilon} ; \mathbb{S}^{2}\right)$, there holds

$$
\frac{1}{\varepsilon^{2}}\left(1-\left|\mathbf{w}_{\varepsilon}\right|^{2}\right)^{2} \leq C\left|\nabla_{\mathrm{s}} \mathbf{w}_{\varepsilon}\right|^{2} \quad \text { pointwise on } M \text {. }
$$


Proof. Thanks to $\left(\mathrm{H}_{1}\right)$, it suffices to show that

$$
\frac{1}{\varepsilon^{2}}\left(1-\left|\widehat{\mathbf{v}}_{\varepsilon}\right|^{2}\right)^{2} \leq C\left|\nabla_{\varepsilon} \widehat{\mathbf{v}}_{\varepsilon}\right|^{2} \quad \text { on } \widehat{M}_{\varepsilon}
$$

Let $T \in \mathcal{T}_{\varepsilon}$ be a triangle with vertices $i_{0}, i_{1}, i_{2}$. Any point $x \in T$ can be written as $x=i_{0}+\lambda_{1}\left(i_{1}-i_{0}\right)+\lambda_{2}\left(i_{2}-i_{0}\right)$, where $\lambda_{1}, \lambda_{2}$ are positive numbers such that $\lambda_{1}+\lambda_{2} \leq 1$. Using the definition of affine interpolant and that $\left|\mathbf{v}_{\varepsilon}\left(i_{0}\right)\right|=1$, we obtain that

$$
1-\left|\widehat{\mathbf{v}}_{\varepsilon}(x)\right| \leq\left|\widehat{\mathbf{v}}_{\varepsilon}(x)-\mathbf{v}_{\varepsilon}\left(i_{0}\right)\right| \leq \sum_{k=1}^{2} \lambda_{k}\left|\mathbf{v}_{\varepsilon}\left(i_{k}\right)-\mathbf{v}_{\varepsilon}\left(i_{0}\right)\right|,
$$

whence, using that $\left|\widehat{\mathbf{v}}_{\varepsilon}\right| \leq 1$ and that $\nabla_{\varepsilon} \widehat{\mathbf{v}}_{\varepsilon}$ is constant on $T$, we deduce

$$
\begin{aligned}
\left(1-\left|\widehat{\mathbf{v}}_{\varepsilon}(x)\right|^{2}\right)^{2} \leq 4\left(1-\left|\widehat{\mathbf{v}}_{\varepsilon}(x)\right|\right)^{2} & \leq 8 \sum_{k=1}^{2}\left|\mathbf{v}_{\varepsilon}\left(i_{k}\right)-\mathbf{v}_{\varepsilon}\left(i_{0}\right)\right|^{2} \\
& =8 \sum_{k=1}^{2}\left|\nabla_{\varepsilon} \widehat{\mathbf{v}}_{\varepsilon}(x)\left(i_{k}-i_{0}\right)\right|^{2} .
\end{aligned}
$$

Now, (39) follows because $\left|i_{k}-i_{0}\right| \leq C \varepsilon$, due to $\left(\mathrm{H}_{2}\right)$.

As a consequence of Lemmas 3 and 5 , if both $\left(\mathrm{H}_{2}\right)$ and $\left(\mathrm{H}_{3}\right)$ are satisfied, then we have

$$
\frac{1}{\varepsilon^{2}} \int_{\widehat{M}_{\varepsilon}}\left(1-\left|\mathbf{w}_{\varepsilon}\right|^{2}\right)^{2} \leq C X Y_{\varepsilon}\left(\mathbf{v}_{\varepsilon}\right)
$$

Another immediate albeit important consequence of the Lemmas above is that for discrete sequences $\mathbf{v}_{\varepsilon}$ with equi-bounded $X Y_{\varepsilon}$-energy with respect to $\varepsilon$, the corresponding vector field $\mathbf{w}_{\varepsilon}$ strongly converges in $L^{2}$ to a unit norm tangent vector field $\mathbf{v}$. This strong convergence follows from the fact that, thanks to Lemma 2 for $\varepsilon$ sufficiently small, the $W_{\varepsilon}^{1,2}$-norm of $\mathbf{w}_{\varepsilon}$ is equivalent to the sum of the $W^{1,2}$-norms of the components of $\mathbf{w}_{\varepsilon}$ with respect to the standard basis of $\mathbb{R}^{3}$. Consequently, the strong convergence in $L^{2}$ follows from standard compactness results for Sobolev spaces on manifolds (see, e.g., [5]) applied component-wise. Then, the unit-norm constraint and the fact that $\mathbf{v}$ is tangent to $M$ follows by passing to the limit in Lemma 4 and Lemma 5 .

\subsection{Jacobians of vector fields on $M$}

In this section, we define the Jacobian determinant of a vector field in the sense of distributions, and we recall a few useful properties. This notion was introduced in the context of Ginzburg-Landau functionals (see e.g. [28]) and in nonlinear elasticity (see e.g. [6], [34]). As we are dealing with vector fields over a manifold, it will be useful to recast the theory in the language of differential forms. 
Given a map $\mathbf{u} \in\left(W^{1,1} \cap L^{\infty}\right)\left(M ; \mathbb{R}^{3}\right)$, we define the "pre-jacobian" of $\mathbf{u}$ (also known as vorticity or current) as the 1 -form

$$
\jmath(\mathbf{u}):=(\gamma, \mathbf{u} \wedge \mathrm{d} \mathbf{u}) .
$$

More explicitely, $g(\mathbf{u})$ is defined via its action on a smooth, tangent field $\mathbf{w}$ on $M$ :

$$
\langle\jmath(\mathbf{u}), \mathbf{w}\rangle=\left(\gamma, \mathbf{u} \times \nabla_{\mathbf{w}} \mathbf{u}\right) .
$$

We can equivalently replace $\nabla_{\mathbf{w}} \mathbf{u}$ with the covariant derivative $\mathrm{D}_{\mathbf{w}} \mathbf{u}$ since the scalar product in (42) does not depend on the component of $\nabla_{\mathbf{w}} \mathbf{u}$ in the direction of $\gamma$.

Suppose now that $\mathbf{u} \in W^{1,1}\left(M ; \mathbb{R}^{3}\right)$ is a unit, tangent field on $M$ (that is, $|\mathbf{u}|=1$ and $\mathbf{u} \cdot \gamma=0$ a.e. $)$, and let $\left(\mathbf{e}_{1}, \mathbf{e}_{2}\right)$ be a local orthonormal basis for the tangent frame of $M$. Then, we have

$$
|\jmath(\mathbf{u})|^{2}=\sum_{k=1}^{2}\left|\mathbf{u} \times \mathrm{D}_{\mathbf{e}_{k}} \mathbf{u}\right|^{2}=\sum_{k=1}^{2}\left|\mathrm{D}_{\mathbf{e}_{k}} \mathbf{u}\right|^{2}=|\mathrm{D} \mathbf{u}|^{2}
$$

where we denote by $|\cdot|$ both the norm on the tangent space and the induced norm on the cotangent space. Moreover, we can write locally that

$$
\mathbf{u}=(\cos \alpha) \mathbf{e}_{1}+(\sin \alpha) \mathbf{e}_{2}
$$

for some scalar function $\alpha$ with bounded variation (this follows, e.g., by [21]). A formal computation shows that

$$
\jmath(\mathbf{u})=\mathrm{d} \alpha-\mathbf{A}
$$

where $\mathbf{A}$, called spin connection, is the 1 -form defined by $\langle\mathbf{A}, \mathbf{w}\rangle:=\mathbf{e}_{1} \cdot \nabla_{\mathbf{w}} \mathbf{e}_{2}$. Note that $\mathbf{A}$ depends on the choice of the frame, but its differential is an intrinsic quantity:

$$
\mathrm{d} \mathbf{A}=G \mathrm{~d} S
$$

where we recall that $G$ is the Gauss curvature of $M$.

The differential $\mathrm{d} \jmath(\mathbf{u})$ will play an important rôle. Since $\mathrm{d} \jmath(\mathbf{u})$ is a 2 -form, it can be written uniquely as $\mathrm{d} \jmath(\mathbf{u})=f \boldsymbol{\omega}$ where $f \in \mathscr{D}^{\prime}(M)$ is scalar and $\boldsymbol{\omega}$ is the volume form on $M$; we use the notation $\star \mathrm{d} \jmath(\mathbf{u}):=f$. In case $M=\mathbb{R}^{2}$ (embedded as the $x y$-plane in $\mathbb{R}^{3}$, so that $\gamma=\mathbf{e}_{3}$ ) and $\mathbf{u}$ is a smooth vector field $\mathbb{R}^{2} \rightarrow \mathbb{R}^{2}$, we easily compute that

$$
\star \mathrm{d} \jmath(\mathbf{u})=2 \operatorname{det} \nabla \mathbf{u},
$$

thus $\star \mathrm{d} \jmath(\mathbf{u})$ can be thought as a generalization of the Jacobian determinant of $\mathbf{u}$, up to a constant factor 2 . If $\mathbf{u}$ is a unit, tangent field on $M$, then by differentiating (45) we see that $\star \mathrm{d} \jmath(\mathbf{u})$ contains topological information about the singularities of $\mathbf{u}$. Recall that $\operatorname{ind}\left(\mathbf{u}, x_{i}\right)$ denotes the local degree of $\mathbf{u}$ at the point $x_{i}$ (see Section 2). 
Lemma 6 Let $\mathbf{u} \in W_{\mathrm{tan}}^{1,1}\left(M ; \mathbb{S}^{2}\right)$ be a unit, tangent field. Suppose that there exist a finite number of points $x_{1}, \ldots, x_{p}$ such that

$$
\mathbf{u} \in W_{\mathrm{loc}}^{1,2}\left(M \backslash\left\{x_{1}, \ldots, x_{p}\right\} ; \mathbb{R}^{3}\right) .
$$

Then

$$
\star \mathrm{d} \jmath(\mathbf{u})=2 \pi \sum_{i=1}^{p} \operatorname{ind}\left(\mathbf{u}, x_{i}\right) \delta_{x_{i}}-G \quad \text { in } \mathscr{D}^{\prime}(M) .
$$

Proof. We can assume without loss of generality that $\mathbf{u}$ is smooth on $M \backslash$ $\left\{x_{1}, \ldots, x_{p}\right\}$, as smooth unit-norm tangent fields are dense in $W^{1,2}$. This follows essentially from the density result [44, Proposition p. 267]. The paper [44] is concerned with the study of maps from $M$ to a fixed target manifold; however, the arguments can be adapted to sections of the unit tangent bundle (see also [18, Section 3] for further details).

For a fixed $i$, take a test function $\varphi \in C^{\infty}(M)$ whose support is simply connected and does not contain any singularity of $\mathbf{v}$ other than $x_{i}$. Suppose that an orthonormal tangent frame $\left(\mathbf{e}_{1}, \mathbf{e}_{2}\right)$ is defined on the support of $\varphi$. Then, we can locally define an angular variable $\alpha$ which satisfies Equation (44) and is smooth, except for a jump across a smooth ray starting at the point $x_{i}$. The size of the jump is constant along the ray, and equal to $2 \pi \operatorname{ind}\left(\mathbf{u}, x_{i}\right)$. The Lebesgue-absolutely continuous part $\mathrm{d}^{\mathrm{ac}} \alpha$ of the differential $\mathrm{d} \alpha$ is actually continuous across the jump, and satisfies (analogously to (45))

$$
\jmath(\mathbf{u})=\mathrm{d}^{\mathrm{ac}} \alpha-\mathbf{A}
$$

on the support of $\varphi$. Thanks to (47), (43) and the fact that $\mathbf{u} \in W^{1,1}$, we deduce that $\mathrm{d}^{\text {ac }} \alpha \in L^{1}$. Moreover, one checks that $\mathrm{d}\left(\mathrm{d}^{\text {ac }} \alpha\right)=0$ on $M \backslash\left\{x_{i}\right\}$.

Now, we compute $\star d\left(\mathrm{~d}^{\text {ac }} \alpha\right)$ in the sense of distributions. For any $\delta>0$, we have

$$
\begin{aligned}
-\left\langle\mathrm{d}^{\mathrm{ac}} \alpha, \star \mathrm{d} \varphi\right\rangle_{L^{2}\left(M \backslash B_{\delta}\left(x_{i}\right)\right)} & =\int_{M \backslash B_{\delta}\left(x_{i}\right)} \mathrm{d}^{\mathrm{ac}} \alpha \wedge \mathrm{d} \varphi=-\int_{M \backslash B_{\delta}\left(x_{i}\right)} \mathrm{d}\left(\mathrm{d}^{\mathrm{ac}} \alpha \wedge \varphi\right) \\
& =\int_{\partial B_{\delta}\left(x_{i}\right)} \mathrm{d}^{\mathrm{ac}} \alpha \wedge \varphi
\end{aligned}
$$

On the other hand, we have

$$
\left|\int_{\partial B_{\delta}\left(x_{i}\right)} \mathrm{d}^{\mathrm{ac}} \alpha \wedge(\varphi-\varphi(0))\right| \leq \delta\left\|\nabla_{\mathrm{s}} \varphi\right\|_{L^{\infty}(M)} \int_{\partial B_{\delta}\left(x_{i}\right)}\left|\mathrm{d}^{\mathrm{ac}} \alpha\right| \mathrm{d} s .
$$

We claim that the right-hand side of (49) converges to 0 at least along a subsequence $\delta_{j} \searrow 0$. For otherwise, there would exist positive numbers $\eta$ and $\delta_{0}$ such that

$$
\delta \int_{\partial B_{\delta}\left(x_{i}\right)}\left|\mathrm{d}^{\mathrm{ac}} \alpha\right| \mathrm{d} s \geq \eta
$$


for any $0<\delta \leq \delta_{0}$. Dividing by $\delta$ both sides of this inequality and integrating over $\delta \in\left(0, \delta_{0}\right)$, we would obtain

$$
\int_{B_{\delta_{0}}\left(x_{i}\right)}\left|\mathrm{d}^{\mathrm{ac}} \alpha\right| \mathrm{d} S \geq \eta \int_{0}^{\delta_{0}} \frac{\mathrm{d} \delta}{\delta}=+\infty,
$$

which is impossible because $\mathrm{d}^{\text {ac }} \alpha \in L^{1}$. Then, we find a subsequence $\delta_{j} \searrow 0$ along which the right-hand side of (49) converges to 0. Taking the limit in (48) along this subsequence, and using (49), we obtain

$$
-\left\langle\mathrm{d}^{\mathrm{ac}} \alpha, \star \mathrm{d} \varphi\right\rangle_{L^{2}(M)}=\lim _{j \rightarrow+\infty} \int_{\partial B_{\delta_{j}}\left(x_{i}\right)} \mathrm{d}^{\mathrm{ac}} \alpha \wedge \varphi(0)=2 \pi \operatorname{ind}\left(\mathbf{u}, x_{i}\right) \varphi(0) .
$$

Since the operator $\star \mathrm{d}$ is $L^{2}(M)$-anti-symmetric, the left-hand side of this identity can be interpreted as the duality pairing $\left\langle\star \mathrm{d}\left(\mathrm{d}^{\mathrm{ac}} \alpha\right), \varphi\right\rangle$, in the sense of distributions. Combining this with (46) and (47), the lemma follows.

Remark 8 Let $\mathbf{u}$ be as in the statement of Lemma 6 . By integrating d $\jmath(\mathbf{u})$ over $M$, and using Stokes' and Gauss-Bonnet's theorems, we deduce

$$
\begin{aligned}
0 & \stackrel{\text { Stokes }}{=} \int_{M} \mathrm{~d} \jmath(\mathbf{u})=\int_{M} \star \mathrm{d} \jmath(\mathbf{u}) \boldsymbol{\omega} \\
& =2 \pi \sum_{i=1}^{p} \operatorname{ind}\left(\mathbf{u}, x_{i}\right)-\int_{M} G \mathrm{~d} S \stackrel{\text { Gauss-Bonnet }}{=} 2 \pi \sum_{i=1}^{p} \operatorname{ind}\left(\mathbf{u}, x_{i}\right)-2 \pi \chi(M) .
\end{aligned}
$$

Therefore, we have $\sum_{i=1}^{p} \operatorname{ind}\left(\mathbf{u}, x_{i}\right)=\chi(M)$, in accordance with the PoincaréHopf theorem.

We define a piecewise-continuous counterpart of $\jmath$. Take a bounded, piecewise-smooth (but not necessarily tangent) map $\mathbf{u}: \widehat{M}_{\varepsilon} \rightarrow \mathbb{R}^{3}$ such that, for any edge $e=[i, j] \in \mathcal{T}_{\varepsilon}^{1}$,

$$
\nabla_{j-i} \mathbf{u}=\nabla \mathbf{u}(j-i) \text { is continuous across } e .
$$

For example, the affine interpolant $\mathbf{u}=\widehat{\mathbf{v}}_{\varepsilon}$ of a discrete field $\mathbf{v} \in \mathrm{T}\left(\mathcal{T}_{\varepsilon} ; \mathbb{S}^{2}\right)$ satisfies (50). We let

$$
\widehat{\jmath}_{\varepsilon}(\mathbf{u}):=\left(\widehat{\gamma}_{\varepsilon}, \mathbf{u} \wedge \mathrm{d} \mathbf{u}\right),
$$

that is the piecewise-smooth 1-form on $\widehat{M}_{\varepsilon}$ satisfying

$$
\left\langle\widehat{\jmath}_{\varepsilon}(\mathbf{u}), \mathbf{w}\right\rangle=\left(\widehat{\gamma}_{\varepsilon}, \mathbf{u} \times \nabla_{\mathbf{w}} \mathbf{u}\right)
$$

for any piecewise-smooth tangent field w on $\widehat{M}_{\varepsilon}$. This form is well-defined and continuous on each triangle of $\widehat{M}_{\varepsilon}$. Note that $\widehat{\jmath}_{\varepsilon}(\mathbf{u})$ may not be continuous across an edge $e=[i, j]$ but $\left\langle\widehat{\jmath}_{\varepsilon}(\mathbf{u}), i-j\right\rangle$ is, therefore the integral of $\widehat{\jmath}_{\varepsilon}(\mathbf{u})$ along $e$ is defined unambiguously. 
4.5 Jacobians of discrete vector-fields

We want to define a notion of "jacobian" for a discrete field $\mathbf{v}_{\varepsilon} \in \mathrm{T}\left(\mathcal{T}_{\varepsilon} ; \mathbb{S}^{2}\right)$ and we have two possibilities: either we apply $\mathrm{d} \widehat{\jmath}_{\varepsilon}$ to the affine interpolant $\widehat{\mathbf{v}}_{\varepsilon}$, or we compute $\mathrm{d} \jmath\left(\mathbf{u}_{\varepsilon}\right)$ for a field $\mathbf{u}_{\varepsilon}: M \rightarrow \mathbb{R}^{3}$ that interpolates $\mathbf{v}_{\varepsilon}$. The first possibility corresponds to the measure

$$
\widehat{\mu}_{\varepsilon}\left(\mathbf{v}_{\varepsilon}\right):=\sum_{T \in \mathcal{T}_{\varepsilon}}\left(\int_{T} \mathrm{~d} \widehat{\jmath}_{\varepsilon}\left(\widehat{\mathbf{v}}_{\varepsilon}\right)\right) \delta_{x_{T}},
$$

where $\delta_{x_{T}}$ is the Dirac delta measure supported by the barycentre $x_{T}$ of $T$. Let $\left(i_{0}, i_{1}, i_{2}\right)$ be the vertices of a triangle $T \in \mathcal{T}_{\varepsilon}$, sorted in counter-clockwise order with respect to the orientation induced by $\gamma$, and let $i_{3}:=i_{0}$. Using Stokes' theorem and the definition of the affine interpolant, we compute

$$
\begin{aligned}
\widehat{\mu}_{\varepsilon}\left(\mathbf{v}_{\varepsilon}\right)[T] & =\sum_{k=0}^{2} \int_{\left[i_{k}, i_{k+1}\right]}\left(\widehat{\gamma}_{\varepsilon}, \widehat{\mathbf{v}}_{\varepsilon} \times \nabla_{i_{k+1}-i_{k}} \widehat{\mathbf{v}}_{\varepsilon}\right) \mathrm{d} s \\
& =\sum_{k=0}^{2}\left(\frac{\gamma\left(i_{k}\right)+\gamma\left(i_{k+1}\right)}{2}, \mathbf{v}_{\varepsilon}\left(i_{k}\right) \times \mathbf{v}_{\varepsilon}\left(i_{k+1}\right)\right)
\end{aligned}
$$

As for the second approach, we construct a suitable field $\mathbf{u}_{\varepsilon}$ in the following way. We fix a sequence $\left(t_{\varepsilon}\right)_{\varepsilon>0}$ such that

$$
\frac{\varepsilon|\log \varepsilon|}{t_{\varepsilon}} \rightarrow 0 \quad \text { as } \varepsilon \rightarrow 0,
$$

e.g. $t_{\varepsilon}:=\varepsilon|\log \varepsilon|^{2}$. Now, reminding that $\mathbf{w}_{\varepsilon}:=\widehat{\mathbf{v}}_{\varepsilon} \circ \widehat{P}_{\varepsilon}^{-1}$, for $x \in M$ we define

$$
\tilde{\mathbf{u}}_{\varepsilon}(x):=\operatorname{proj}_{\mathrm{T}_{x} M} \mathbf{w}_{\varepsilon}(x) \quad \text { and } \quad \mathbf{u}_{\varepsilon}(x):=\eta_{\varepsilon}\left(\left|\tilde{\mathbf{u}}_{\varepsilon}(x)\right|\right) \tilde{\mathbf{u}}_{\varepsilon}(x),
$$

where $\eta_{\varepsilon}(s):=\min \left\{t_{\varepsilon}^{-1}, s^{-1}\right\}$. Note that $\mathbf{u}_{\varepsilon}$ is a Lipschitz tangent field on $M$ and $\mathbf{u}_{\varepsilon}=\mathbf{v}_{\varepsilon}$ on $\mathcal{T}_{\varepsilon}^{0}$. Next, we set

$$
\mu_{\varepsilon}\left(\mathbf{v}_{\varepsilon}\right):=\sum_{T \in \mathcal{T}_{\varepsilon}}\left(\int_{P(T)} \mathrm{d} \jmath\left(\mathbf{u}_{\varepsilon}\right)\right) \delta_{P\left(x_{T}\right)} .
$$

Given a Borel set $E \subseteq M$, let $E_{\varepsilon}$ be the union of all the $P(T)$ 's such that $T \in$ $\mathcal{T}_{\varepsilon}, P\left(x_{T}\right) \in E$. If $\left|\tilde{\mathbf{u}}_{\varepsilon}\right| \geq 1 / 4$ on $\partial E_{\varepsilon}$, then we can find a unit tangent field $\mathbf{U}_{\varepsilon} \in$ $W_{\tan }^{1,1}\left(E_{\varepsilon} ; \mathbb{S}^{2}\right)$ such that $\mathbf{U}_{\varepsilon}=\mathbf{u}_{\varepsilon}$ on $\partial E_{\varepsilon}$ and $\mathbf{U}_{\varepsilon}$ is smooth except at finitely many points. (One can modify $\tilde{\mathbf{u}}_{\varepsilon}$ in such a way that it is smooth and has 0 as a regular value, then define $\mathbf{U}_{\varepsilon}:=\tilde{\mathbf{u}}_{\varepsilon} /\left|\tilde{\mathbf{u}}_{\varepsilon}\right|$.) Since $\mu_{\varepsilon}\left(\mathbf{v}_{\varepsilon}\right)[E]=\int_{E_{\varepsilon}} \mathrm{d} \jmath\left(\mathbf{u}_{\varepsilon}\right)$ and, by Stokes' theorem, the latter only depends on the restriction of $\mathbf{u}_{\varepsilon}$ to $\partial E_{\varepsilon}$, we have $\mu\left(\mathbf{v}_{\varepsilon}\right)[E]=\int_{E_{\varepsilon}} \mathrm{d} \jmath\left(\mathbf{U}_{\varepsilon}\right)$ and hence, by Lemma 6 ,

$$
\mu_{\varepsilon}\left(\mathbf{v}_{\varepsilon}\right)[E]=2 \pi \operatorname{ind}\left(\mathbf{u}_{\varepsilon}, E_{\varepsilon}\right)-\int_{E_{\varepsilon}} G \mathrm{~d} S .
$$


In this sense, the measure $\mu_{\varepsilon}\left(\mathbf{v}_{\varepsilon}\right)$ can be thought of as a generalization of the discrete vorticity defined in [2] (see in particular [2, Remark 2.1]), and immediately provides information on the "topological" behaviour of $\mathbf{v}_{\varepsilon}$. On the other hand, the measure $\widehat{\mu}_{\varepsilon}\left(\mathbf{v}_{\varepsilon}\right)$ has the advantage of being simpler to evaluate, thanks to (53). Luckily, if the XY-energy of the field $\mathbf{v}_{\varepsilon}$ satisfies a logarithmic bound, then the two measures are close to each other.

Proposition 2. Suppose that $\left(\mathrm{H}_{1}\right),\left(\mathrm{H}_{2}\right),\left(\mathrm{H}_{3}\right)$ are satisfied. Let $\left(\mathbf{v}_{\varepsilon}\right)_{0<\varepsilon \leq \varepsilon_{0}}$ be a sequence of discrete fields that satisfies $(\mathrm{H})$ for some $\varepsilon$-independent constant $\Lambda$ and any $0<\varepsilon \leq \varepsilon_{0}$. Then, there holds

$$
\left\|\widehat{\mu}_{\varepsilon}\left(\mathbf{v}_{\varepsilon}\right)-\mu_{\varepsilon}\left(\mathbf{v}_{\varepsilon}\right)\right\|_{\text {flat }} \leq C\left(\frac{\varepsilon|\log \varepsilon|}{t_{\varepsilon}}+\varepsilon|\log \varepsilon|\right) .
$$

In particular, the difference between the two measures converges to zero in the flat norm as $\varepsilon \rightarrow 0$, if we assume that (54) holds. The rest of this section is devoted to the proof of Proposition 2. The key fact is the following continuity property for the Jacobian, which is well-known for maps $\mathbf{u}: \Omega \subseteq \mathbb{R}^{n} \rightarrow \mathbb{R}^{n}$ (see e.g. [3, Lemma 2.1]).

Lemma 7 Let $\mathbf{u}, \mathbf{w}$ be (not necessarily tangent) fields in $W^{1,2}\left(M ; \mathbb{R}^{3}\right)$. Then, there holds

$$
\begin{gathered}
\|\star \mathrm{d} \jmath(\mathbf{u})\|_{L^{1}(M)} \leq C\left(\|\mathbf{u}\|_{L^{2}(M)}^{2}+\left\|\nabla_{\mathrm{s}} \mathbf{u}\right\|_{L^{2}(M)}^{2}\right), \\
\|\star \mathrm{d} \jmath(\mathbf{u})-\star \mathrm{d} \jmath(\mathbf{w})\|_{\text {flat }} \leq\|\mathbf{u}-\mathbf{w}\|_{L^{2}(M)}\left(\left\|\nabla_{\mathrm{s}} \mathbf{u}\right\|_{L^{2}(M)}+\left\|\nabla_{\mathrm{s}} \mathbf{w}\right\|_{L^{2}(M)}\right) .
\end{gathered}
$$

Proof. By a density argument, we can assume WLOG that $\mathbf{u}, \mathbf{w}$ are smooth. Using Einstein convention, we can write

$$
\jmath(\mathbf{u})=\gamma^{i} \jmath_{i}(\mathbf{u}), \quad \text { where } \jmath_{i}(\mathbf{u}):=\epsilon_{i j k} \mathbf{u}^{j} \mathrm{~d} \mathbf{u}^{k}
$$

and $\epsilon_{i j k}$ is the Levi-Civita symbol, given by $\epsilon_{i j k}:=1$ if $(i, j, k)$ is an even permutation of $(1,2,3), \epsilon_{i j k}:=-1$ if it is an odd permutation, and $\epsilon_{i j k}:=0$ otherwise. By differentiating, we deduce

$$
\mathrm{d} \jmath(\mathbf{u})=\epsilon_{i j k} \mathbf{u}^{j} \mathrm{~d} \boldsymbol{\gamma}^{i} \wedge \mathrm{d} \mathbf{u}^{k}+\epsilon_{i j k} \boldsymbol{\gamma}^{i} \mathrm{~d} \mathbf{u}^{j} \wedge \mathrm{d} \mathbf{u}^{k},
$$

whence (58) immediately follows by applying the Hölder inequality and using that $\left|\nabla_{\mathrm{s}} \gamma\right|$ is bounded. We now prove (59). A straightforward computation shows that

$$
\jmath_{3}(\mathbf{u})-\jmath_{3}(\mathbf{w})=\frac{1}{2}\left(\jmath_{3}\left(\mathbf{u}^{1}-\mathbf{w}^{1}, \mathbf{u}^{2}+\mathbf{w}^{2}\right)-\jmath_{3}\left(\mathbf{u}^{2}-\mathbf{w}^{2}, \mathbf{u}^{1}+\mathbf{w}^{1}\right)\right)
$$

and similar equalities hold for $\jmath_{1}, \jmath_{2}$, therefore

$$
\mathrm{d} \jmath(\mathbf{u})-\mathrm{d} \jmath(\mathbf{w})=\frac{\epsilon_{i j k}}{2} \mathrm{~d}\left(\gamma^{i} \jmath_{i}\left(\mathbf{u}^{j}-\mathbf{w}^{j}, \mathbf{u}^{k}+\mathbf{w}^{k}\right)\right) .
$$


Now, fix a function $\varphi \in C_{\mathrm{c}}^{\infty}(U)$. Thanks to (61) and an integration by parts, we deduce

$$
\langle\star \mathrm{d} \jmath(\mathbf{u})-\star \mathrm{d} \jmath(\mathbf{w}), \varphi\rangle=-\frac{\epsilon_{i j k}}{2}\left\langle\gamma^{i} \jmath_{i}\left(\mathbf{u}^{j}-\mathbf{w}^{j}, \mathbf{u}^{k}+\mathbf{w}^{k}\right), \star \mathrm{d} \varphi\right\rangle .
$$

The definition of $\jmath_{i}$ and the Hölder inequality immediately imply

$$
\langle\star \mathrm{d} \jmath(\mathbf{u})-\star \mathrm{d} \jmath(\mathbf{w}), \varphi\rangle \leq\|\mathbf{u}-\mathbf{w}\|_{L^{2}(M)}\left\|\nabla_{\mathrm{s}} \mathbf{u}-\nabla_{\mathrm{s}} \mathbf{w}\right\|_{L^{2}(M)}\left\|\nabla_{\mathrm{s}} \varphi\right\|_{L^{\infty}(M)},
$$

whence (59) follows by taking the supremum over $\varphi$.

Lemma 7 has a counterpart in the piecewise-continuous setting. For further reference, here we only mention that

Lemma 8 Let $\mathbf{u}: \widehat{M}_{\varepsilon} \rightarrow \mathbb{R}^{3}$ be a (not necessarily tangent) piecewise-smooth field that satisfies (50). Then, there holds

$$
\left\|\star \mathrm{d} \widehat{\jmath}_{\varepsilon}(\mathbf{u})\right\|_{L^{1}(T)} \leq C\left(\|\mathbf{u}\|_{L^{2}(T)}^{2}+\left\|\nabla_{\varepsilon} \mathbf{u}\right\|_{L^{2}(T)}^{2}\right) \quad \text { for any } T \in \mathcal{T}_{\varepsilon} .
$$

Proof. We argue as in Lemma 7, using that the functions $\widehat{\gamma}_{\varepsilon}$ are Lipschitz continuous and $\left\|\nabla_{\varepsilon} \widehat{\gamma}_{\varepsilon}\right\|_{L^{\infty}\left(\widehat{M}_{\varepsilon}\right)} \leq\left\|\nabla_{\mathrm{s}} \gamma\right\|_{L^{\infty}(M)}$.

Proof of Proposition 2. Given a piecewise-smooth map $\mathbf{u}: \widehat{M}_{\varepsilon} \rightarrow \mathbb{R}^{3}$, we let $\jmath(\mathbf{u}):=(\gamma \circ P, \mathbf{u} \wedge \mathrm{d} \mathbf{u})$, i.e. we extend the operator $\mathbf{u} \mapsto \jmath(\mathbf{u})$ to fields $\mathbf{u}$ that are not defined on $M$ by pre-composing $\gamma$ with the projection $P: U \rightarrow M$. When $\mathbf{u}$ is a piecewise-smooth field, we denote by $\mathrm{d} \jmath(\mathbf{u}), \mathrm{d} \widehat{\jmath}_{\varepsilon}(\mathbf{u})$ the Lebesgue-absolutely continuous part of the distributional differential of $\jmath(\mathbf{u}), \widehat{\jmath}_{\varepsilon}(\mathbf{u})$ respectively that is, we neglect any jumps that may arise at the boundary of the regions where $\mathbf{u}$ is smooth.

Let $\left(\mathbf{v}_{\varepsilon}\right)$ be a sequence of discrete fields satisfying the energy bound $(\mathrm{H})$. The assumption (H) together with (13), (40) and the fact that $\left|\widehat{\mathbf{v}}_{\varepsilon}\right| \leq 1$ implies that

$$
\left\|\widehat{\mathbf{v}}_{\varepsilon}\right\|_{L^{2}\left(\widehat{M}_{\varepsilon}\right)}^{2}+\left\|\nabla_{\varepsilon} \widehat{\mathbf{v}}_{\varepsilon}\right\|_{L^{2}\left(\widehat{M}_{\varepsilon}\right)}^{2}+\varepsilon^{-2}\left\|1-\left|\widehat{\mathbf{v}}_{\varepsilon}\right|^{2}\right\|_{L^{2}\left(\widehat{M}_{\varepsilon}\right)}^{2} \leq C|\log \varepsilon|
$$

for any $0<\varepsilon \leq \varepsilon_{0}$ and some constant $C=C\left(M, \Lambda, \varepsilon_{0}\right)$, provided that $\varepsilon_{0}<1$. We decompose the difference $\widehat{\mu}_{\varepsilon}\left(\mathbf{v}_{\varepsilon}\right)-\mu_{\varepsilon}\left(\mathbf{v}_{\varepsilon}\right)$ as a sum of several terms:

$$
\begin{aligned}
\widehat{\mu}_{\varepsilon}\left(\mathbf{v}_{\varepsilon}\right)-\mu_{\varepsilon}\left(\mathbf{v}_{\varepsilon}\right) & =\underbrace{\widehat{\mu}_{\varepsilon}\left(\mathbf{v}_{\varepsilon}\right)-\star \mathrm{d} \widehat{\jmath}_{\varepsilon}\left(\widehat{\mathbf{v}}_{\varepsilon}\right)}_{=: A_{1}}+\underbrace{\star \mathrm{d} \widehat{\jmath}_{\varepsilon}\left(\widehat{\mathbf{v}}_{\varepsilon}\right)-\star \mathrm{d} \jmath\left(\widehat{\mathbf{v}}_{\varepsilon}\right)}_{=: A_{2}} \\
& +\underbrace{\star \mathrm{d} \jmath\left(\widehat{\mathbf{v}}_{\varepsilon}\right)-\star \mathrm{d} \jmath\left(\mathbf{w}_{\varepsilon}\right)}_{=: A_{3}}+\underbrace{\star \mathrm{d} \jmath\left(\mathbf{w}_{\varepsilon}\right)-\star \mathrm{d} \jmath\left(\tilde{\mathbf{u}}_{\varepsilon}\right)}_{=: A_{4}} \\
& +\underbrace{\star \mathrm{d} \jmath\left(\tilde{\mathbf{u}}_{\varepsilon}\right)-\star \mathrm{d} \jmath\left(\mathbf{u}_{\varepsilon}\right)}_{=: A_{5}}+\underbrace{\star \mathrm{d} \jmath\left(\mathbf{u}_{\varepsilon}\right)-\mu_{\varepsilon}\left(\mathbf{u}_{\varepsilon}\right)}_{=: A_{6}} .
\end{aligned}
$$

Throughout the rest of the proof, we let $\varphi \in C_{\mathrm{c}}^{\infty}(U)$ be an arbitrarily fixed test function. 
Analysis of $A_{1}$. There holds

$$
\begin{aligned}
\left\langle\widehat{\mu}_{\varepsilon}\left(\mathbf{v}_{\varepsilon}\right)-\star \mathrm{d} \widehat{\jmath}_{\varepsilon}\left(\widehat{\mathbf{v}}_{\varepsilon}\right), \varphi\right\rangle & =\sum_{T \in \mathcal{T}_{\varepsilon}} \int_{T}\left(\varphi\left(x_{T}\right)-\varphi\right) \mathrm{d} \widehat{\jmath}_{\varepsilon}\left(\widehat{\mathbf{v}}_{\varepsilon}\right) \\
& \leq\left\|\star \mathrm{d} \widehat{\jmath}_{\varepsilon}\left(\widehat{\mathbf{v}}_{\varepsilon}\right)\right\|_{L^{1}\left(\widehat{M}_{\varepsilon}\right)}\|\nabla \varphi\|_{L^{\infty}(U)} \sup _{T \in \mathcal{T}_{\varepsilon}} \operatorname{diam}(T)
\end{aligned}
$$

Using Lemma 8, the assumption $\left(\mathrm{H}_{2}\right)$ and (62), we deduce

$$
\left\|\widehat{\mu}_{\varepsilon}\left(\mathbf{v}_{\varepsilon}\right)-\star \mathrm{d} \widehat{\jmath}_{\varepsilon}\left(\widehat{\mathbf{v}}_{\varepsilon}\right)\right\|_{\text {flat }} \leq C \varepsilon|\log \varepsilon| .
$$

Analysis of $A_{2}$. By integrating by parts on each triangle of the triangulation, we can write

$$
\left\langle\star \mathrm{d} \widehat{\jmath}_{\varepsilon}\left(\widehat{\mathbf{v}}_{\varepsilon}\right)-\star \mathrm{d} \jmath\left(\widehat{\mathbf{v}}_{\varepsilon}\right), \varphi\right\rangle=-\sum_{T \in \mathcal{T}_{\varepsilon}} \int_{T}\left(\widehat{\jmath}_{\varepsilon}\left(\widehat{\mathbf{v}}_{\varepsilon}\right)-\jmath\left(\widehat{\mathbf{v}}_{\varepsilon}\right)\right) \wedge(\star \mathrm{d} \varphi) .
$$

The total contribution of the boundary terms vanishes, because each edge appears in the sum twice, with opposite orientations. Then, by the Hölder inequality, we obtain

$$
\left\|\star \mathrm{d} \widehat{\jmath}_{\varepsilon}\left(\widehat{\mathbf{v}}_{\varepsilon}\right)-\star \mathrm{d} \jmath\left(\widehat{\mathbf{v}}_{\varepsilon}\right)\right\|_{\text {flat }} \leq\left\|\widehat{\gamma}_{\varepsilon}-\gamma \circ P\right\|_{L^{\infty}\left(\widehat{M}_{\varepsilon}\right)}\left\|\widehat{\mathbf{v}}_{\varepsilon}\right\|_{L^{2}\left(\widehat{M}_{\varepsilon}\right)}\left\|\nabla_{\varepsilon} \widehat{\mathbf{v}}_{\varepsilon}\right\|_{L^{2}\left(\widehat{M}_{\varepsilon}\right)} .
$$

Using that $\left|\widehat{\mathbf{v}}_{\varepsilon}\right| \leq 1$, that $\left\|\widehat{\gamma}_{\varepsilon}-\gamma \circ P\right\|_{L^{\infty}\left(\widehat{M}_{\varepsilon}\right)} \leq C \varepsilon$ (as a consequence of $\left.\left(\mathrm{H}_{2}\right)\right)$ and (62), we conclude that

$$
\left\|\star \mathrm{d} \widehat{\jmath}_{\varepsilon}\left(\widehat{\mathbf{v}}_{\varepsilon}\right)-\star \mathrm{d} \jmath\left(\widehat{\mathbf{v}}_{\varepsilon}\right)\right\|_{\text {flat }} \leq C \varepsilon|\log \varepsilon|^{1 / 2} .
$$

Analysis of $A_{3}$. Set $\omega:=\jmath\left(\mathbf{w}_{\varepsilon}\right)$, so $\omega$ is a 1 -form on $M$ and $\widehat{P}_{\varepsilon}^{*}(\omega)=\jmath\left(\widehat{\mathbf{v}}_{\varepsilon}\right)$. Since the sets $\widehat{P}_{\varepsilon}(T)$ for $T \in \mathcal{T}_{\varepsilon}$ define a Borel partition of $M$ up to sets of measure zero, we can write

$$
\left\langle\star \mathrm{d} \jmath\left(\mathbf{w}_{\varepsilon}\right)-\star \mathrm{d} \jmath\left(\widehat{\mathbf{v}}_{\varepsilon}\right), \varphi\right\rangle=\sum_{T \in \mathcal{T}_{\varepsilon}}\left(\int_{\widehat{P}_{\varepsilon}(T)} \varphi \mathrm{d} \omega-\int_{T} \varphi \widehat{P}_{\varepsilon}^{*}(\mathrm{~d} \omega)\right) .
$$

Thanks to the assumption $\left(\mathrm{H}_{1}\right), \widehat{P}_{\varepsilon}$ induces a bilipschitz equivalence of $T$ onto its image. Therefore, by applying the area formula to the first integral in the right-hand side, we deduce

$$
\begin{aligned}
\left\langle\star \mathrm{d} \jmath\left(\mathbf{w}_{\varepsilon}\right)-\star \mathrm{d} \jmath\left(\widehat{\mathbf{v}}_{\varepsilon}\right), \varphi\right\rangle & =\sum_{T \in \mathcal{T}_{\varepsilon}} \int_{T}\left(\widehat{P}_{\varepsilon}^{*}(\varphi \mathrm{d} \omega)-\varphi \widehat{P}_{\varepsilon}^{*}(\mathrm{~d} \omega)\right) \\
& =\sum_{T \in \mathcal{T}_{\varepsilon}} \int_{T}\left(\varphi \circ \widehat{P}_{\varepsilon}-\varphi\right) \widehat{P}_{\varepsilon}^{*}(\mathrm{~d} \omega) \\
& =\int_{\widehat{M}_{\varepsilon}}\left(\varphi \circ \widehat{P}_{\varepsilon}-\varphi\right) \mathrm{d} \jmath\left(\widehat{\mathbf{v}}_{\varepsilon}\right) .
\end{aligned}
$$


The Hölder inequality and Lemma 8 then yield

$$
\begin{aligned}
\left\langle\star \mathrm{d} \jmath\left(\mathbf{w}_{\varepsilon}\right)-\star \mathrm{d} \jmath\left(\widehat{\mathbf{v}}_{\varepsilon}\right), \varphi\right\rangle \leq C \operatorname{dist}\left(\widehat{M}_{\varepsilon}, M\right)\left\|\nabla_{\varepsilon} \varphi\right\|_{L^{\infty}\left(\widehat{M}_{\varepsilon}\right)} \\
\cdot\left(\left\|\widehat{\mathbf{v}}_{\varepsilon}\right\|_{L^{2}\left(\widehat{M}_{\varepsilon}\right)}^{2}+\left\|\nabla_{\varepsilon} \widehat{\mathbf{v}}_{\varepsilon}\right\|_{L^{2}\left(\widehat{M}_{\varepsilon}\right)}^{2}\right)
\end{aligned}
$$

whence, by applying $\left(\mathrm{H}_{2}\right)$ and $(62)$, we conclude

$$
\left\|\star \mathrm{d} \jmath\left(\widehat{\mathbf{v}}_{\varepsilon}\right)-\star \mathrm{d} \jmath\left(\mathbf{w}_{\varepsilon}\right)\right\|_{\text {flat }} \leq C \varepsilon|\log \varepsilon| .
$$

Analysis of $A_{4}$. Reminding the definition (55) of $\tilde{\mathbf{u}}_{\varepsilon}$ and Lemma 4, we have

$$
\left\|\mathbf{w}_{\varepsilon}-\tilde{\mathbf{u}}_{\varepsilon}\right\|_{L^{\infty}(M)}=\left\|\left(\mathbf{w}_{\varepsilon}, \gamma\right)\right\|_{L^{\infty}(M)} \leq C \varepsilon .
$$

On the other hand, using (62) and the assumption $\left(\mathrm{H}_{1}\right)$, we compute that

$$
\left\|\nabla_{\mathbf{S}} \mathbf{w}_{\varepsilon}\right\|_{L^{2}(M)}+\left\|\nabla_{\mathbf{S}} \tilde{\mathbf{u}}_{\varepsilon}\right\|_{L^{2}(M)} \leq C|\log \varepsilon|^{1 / 2} .
$$

We combine (59) in Lemma 7 with (66) and (67) to obtain

$$
\left\|\star \mathrm{d} \jmath\left(\mathbf{w}_{\varepsilon}\right)-\star \mathrm{d} \jmath\left(\tilde{\mathbf{u}}_{\varepsilon}\right)\right\|_{\text {flat }} \leq C \varepsilon|\log \varepsilon|^{1 / 2} .
$$

Analysis of $A_{5}$. From the definition (55) of $\mathbf{u}_{\varepsilon}$ and (67), we compute

$$
\left\|\nabla_{\mathrm{S}} \mathbf{u}_{\varepsilon}\right\|_{L^{2}(M)} \leq 2 t_{\varepsilon}^{-1}\left\|\nabla_{\mathrm{s}} \tilde{\mathbf{u}}_{\varepsilon}\right\|_{L^{2}(M)} \leq C t_{\varepsilon}^{-1}|\log \varepsilon|^{1 / 2}
$$

On the other hand, thanks to (66) we obtain

$$
\left|\mathbf{u}_{\varepsilon}-\tilde{\mathbf{u}}_{\varepsilon}\right|^{2} \leq\left(1-\left|\tilde{\mathbf{u}}_{\varepsilon}\right|\right)^{2} \leq\left(1-\left|\mathbf{w}_{\varepsilon}\right|\right)^{2}+C \varepsilon \leq\left(1-\left|\mathbf{w}_{\varepsilon}\right|^{2}\right)^{2}+C \varepsilon .
$$

By integrating both sides of the inequality, and making a change of variable we deduce

$$
\left\|\mathbf{u}_{\varepsilon}-\tilde{\mathbf{u}}_{\varepsilon}\right\|_{L^{2}(M)}^{2} \leq\left\|1-\left|\widehat{\mathbf{v}}_{\varepsilon}\right|^{2}\right\|_{L^{2}\left(\widehat{M}_{\varepsilon}\right)}^{2}+C \varepsilon \mathscr{H}^{2}\left(\widehat{M}_{\varepsilon}\right) \leq C \varepsilon^{2}|\log \varepsilon|
$$

For the last inequality, we have used (62) and the fact that $\mathscr{H}^{2}\left(\widehat{M}_{\varepsilon}\right) \leq C$, which follows from $\left(\mathrm{H}_{1}\right)$. Thus, by applying (59) (Lemma 7), with the help of (67), (69) and (70) we deduce that

$$
\left\|\star \mathrm{d} \jmath\left(\tilde{\mathbf{u}}_{\varepsilon}\right)-\star \mathrm{d} \jmath\left(\mathbf{u}_{\varepsilon}\right)\right\|_{\text {flat }} \leq C t_{\varepsilon}^{-1} \varepsilon|\log \varepsilon| .
$$

Analysis of $A_{6}$. Arguing as in the proof of (63), and using (58) in Lemma 7 instead of Lemma 8, we obtain

$$
\left\|\star \mathrm{d} \jmath\left(\mathbf{u}_{\varepsilon}\right)-\mu_{\varepsilon}\left(\mathbf{v}_{\varepsilon}\right)\right\|_{\text {flat }} \leq \varepsilon|\log \varepsilon| .
$$

Now, the proposition follows by combining (63), (64), (65), (68), (71) and (72). 


\section{The zero-order $\Gamma$-convergence: emergence of defects}

5.1 Localized lower bounds for the energy

Thanks to Proposition 2, the compactness of the sequence $\widehat{\mu}_{\varepsilon}\left(\mathbf{v}_{\varepsilon}\right)$ is equivalent to the compactness of $\mu_{\varepsilon}\left(\mathbf{v}_{\varepsilon}\right)$. The latter is defined in terms of the fields $\mathbf{w}_{\varepsilon} \in W^{1, \infty}\left(M ; \mathbb{R}^{3}\right)$, given by (55), which interpolate continuously the discrete fields $\mathbf{v}_{\varepsilon}$ but are not necessarily tangent nor unit-valued. We discuss now a localized lower bound for the Dirichlet energy of $\mathbf{w}_{\varepsilon}$. Similar results are well-known in the continuum Ginzburg-Landau setting, where they play a major rôle (see, e.g., [27, Theorems 2.1 and 4.1] and [40, Theorem 1]), and are also available for the discrete XY-energy [2, Proposition 3.2]. Given a point $x_{0} \in M$ and a radius $\rho>0$, we denote by $B_{\rho}\left(x_{0}\right)$ the geodesic ball of centre $x_{0}$ and radius $\rho$. We follow the approach in [27]. We define

$$
\alpha_{\varepsilon}:=(1-C \varepsilon)^{-2} \operatorname{essinf}_{x \in M} \inf _{\mathbf{X} \in \mathrm{T}_{x} M,|\mathbf{X}|=1}\left(\mathbf{A}_{\varepsilon}^{-1}(x) \mathbf{X}, \mathbf{X}\right)\left(\operatorname{det} \mathbf{A}_{\varepsilon}(x)\right)^{1 / 2}
$$

and, for $0<\varepsilon<\rho$ and $d \in \mathbb{Z}$, we let

$$
\lambda_{\varepsilon}(\rho, d):=\alpha_{\varepsilon} \min _{0 \leq m \leq 1}\left\{\frac{\pi|d|}{\rho+C \rho^{2}} m^{2} \vee \frac{C}{\varepsilon}(1-m)^{3}\right\} .
$$

The constant $C$, which will be selected below, does not depend on $\varepsilon, \rho$ and $d$. Recall that, given a vector field $\mathbf{v}_{\varepsilon} \in \mathrm{T}\left(\mathcal{T}_{\varepsilon} ; \mathbb{S}^{2}\right)$, we let $\mathbf{w}_{\varepsilon}:=\widehat{\mathbf{v}}_{\varepsilon} \circ \widehat{P}_{\varepsilon}^{-1}$ and we denote by $\mathbf{u}_{\varepsilon}$ the vector field defined by $(55)$.

Lemma 9 There exist positive constants $R_{*}$ and $\varepsilon_{*}$ with the following property: for any $\varepsilon \in\left(0, \varepsilon_{*}\right]$, any $x_{0} \in M$, any $\rho \in\left(\varepsilon, R_{*}\right]$ and any $\mathbf{v}_{\varepsilon} \in \mathrm{T}_{\varepsilon}\left(\mathcal{T}_{\varepsilon} ; \mathbb{S}^{2}\right)$ such that $\left|\mathbf{w}_{\varepsilon}\right| \geq 1 / 2$ on $\partial B_{\rho}\left(x_{0}\right)$, there holds

$$
\frac{1}{2}\left|\mathbf{w}_{\varepsilon}\right|_{W_{\varepsilon}^{1,2}\left(\partial B_{\rho}\left(x_{0}\right)\right)}^{2} \geq \lambda_{\varepsilon}\left(\rho, \operatorname{ind}\left(\mathbf{u}_{\varepsilon}, B_{\rho}\left(x_{0}\right)\right)\right) .
$$

Moreover, there holds

$$
\lambda_{\varepsilon}(\rho, d) \geq \frac{(1-C \varepsilon) \pi|d|}{\rho+C \rho^{2}}-C \varepsilon^{1 / 3}|d|^{1 / 3} \rho^{-4 / 3}
$$

for any $\rho>\varepsilon, d \in \mathbb{Z}$ and some constant $C$ which does not depend on $\varepsilon, \rho, d$.

Proof of Lemma 9. This argument is adapted from [27, Theorem 2.1]. Throughout the proof, the symbol $C$ denotes several constants that do not depend on $\varepsilon$ or $x_{0} \in M$, but possibly on $R_{*}, \varepsilon_{*}$. We start by proving (75).

Step 1. There exist positive numbers $R_{*}$ and $C$ such that, for any $x_{0} \in M$ and any $0<\rho \leq R_{*}$, there holds

$$
\mathscr{H}^{1}\left(\partial B_{\rho}\left(x_{0}\right)\right) \leq 2 \pi \rho+C \rho^{2} .
$$

Indeed, thanks to the area formula, the left-hand side is bounded by $\operatorname{Lip}\left(\exp _{x_{0} \mid D_{\rho}}\right) \mathscr{H}^{1}\left(\partial D_{\rho}\right)$, where $\exp _{x_{0}}: \mathrm{T}_{x_{0}} M \rightarrow M$ is the exponential map 
and $D_{\rho} \subseteq \mathrm{T}_{x_{0}} M$ is the disk of radius $\rho$ centred at the origin. Since $\mathrm{D}\left(\exp _{x_{0}}\right)=$ $\operatorname{Id}_{\mathrm{T}_{x_{0}} M}$, by a Taylor expansion we see that $\operatorname{Lip}\left(\exp _{x_{0} \mid D_{\rho}}\right) \leq 1-C \rho$ if $\rho$ is small enough. By a compactness argument, the numbers $R_{*}$ and $C$ can be chosen uniformly with respect to $x_{0}$.

Step 2. Take a point $x_{0} \in M$ and a field $\mathbf{v}_{\varepsilon} \in T\left(\mathcal{T}_{\varepsilon} ; \mathbb{S}^{2}\right)$ such that $\left|\mathbf{w}_{\varepsilon}\right| \geq 1 / 2$ on $\partial B_{\rho}\left(x_{0}\right)$. Since $x_{0}$ will be fixed for the rest of the proof, we will omit it from the notation. Thanks to Lemma 4 and to (54), (55) we have that $\left|\tilde{\mathbf{u}}_{\varepsilon}\right| \geq 1 / 4$ and $\mathbf{u}_{\varepsilon}=\tilde{\mathbf{u}}_{\varepsilon} /\left|\tilde{\mathbf{u}}_{\varepsilon}\right|$ on $\partial B_{\rho}$ provided that $\varepsilon$ is small enough, say, smaller that some number $\varepsilon_{*}$. Then, we compute

$$
\begin{aligned}
\left|\mathbf{w}_{\varepsilon}\right|\left|\left(\boldsymbol{\gamma}, \mathbf{u}_{\varepsilon} \times \nabla_{\boldsymbol{\tau}} \mathbf{u}_{\varepsilon}\right)\right| & =\frac{\left|\mathbf{w}_{\varepsilon}\right|}{\left|\tilde{\mathbf{u}}_{\varepsilon}\right|^{2}}\left|\left(\boldsymbol{\gamma}, \tilde{\mathbf{u}}_{\varepsilon} \times \nabla_{\boldsymbol{\tau}} \tilde{\mathbf{u}}_{\varepsilon}\right)\right| \\
& =\frac{\left|\mathbf{w}_{\varepsilon}\right|}{\left|\tilde{\mathbf{u}}_{\varepsilon}\right|^{2}}\left|\left(\boldsymbol{\gamma}, \mathbf{w}_{\varepsilon} \times \nabla_{\boldsymbol{\tau}} \mathbf{w}_{\varepsilon}\right)-\left(\mathbf{w}_{\varepsilon}, \gamma\right)\left(\gamma, \mathbf{w}_{\varepsilon} \times \nabla_{\boldsymbol{\tau}} \gamma\right)\right| \\
& \leq \frac{\left|\mathbf{w}_{\varepsilon}\right|^{2}}{\left|\tilde{\mathbf{u}}_{\varepsilon}\right|^{2}}\left(\left|\nabla_{\mathrm{s}} \mathbf{w}_{\varepsilon}\right|+C\left|\left(\mathbf{w}_{\varepsilon}, \gamma\right)\right|\right) .
\end{aligned}
$$

Using (55) and the fact that $\left|\tilde{\mathbf{u}}_{\varepsilon}\right| \geq 1 / 4$, we can bound the ratio $\left|\mathbf{w}_{\varepsilon}\right| /\left|\tilde{\mathbf{u}}_{\varepsilon}\right|$ in terms of $\left|\left(\mathbf{w}_{\varepsilon}, \gamma\right)\right|$, which in turns is bounded by $C \varepsilon$, due to Lemma 4 . This yields

$$
\left|\mathbf{w}_{\varepsilon}\right|\left|\left(\boldsymbol{\gamma}, \mathbf{u}_{\varepsilon} \times \nabla_{\boldsymbol{\tau}} \mathbf{u}_{\varepsilon}\right)\right| \leq(1+C \varepsilon)\left(\left|\nabla_{\mathrm{s}} \mathbf{w}_{\varepsilon}\right|+C \varepsilon\right) .
$$

After a rearrangement, and using again that $\left|\mathbf{w}_{\varepsilon}\right| \geq 1 / 2$, we obtain

$$
\left|\nabla_{\mathbf{s}} \mathbf{w}_{\varepsilon}\right|^{2} \geq(1+C \varepsilon)^{-2}\left|\mathbf{w}_{\varepsilon}\right|^{2}\left(\left|\left(\boldsymbol{\gamma}, \mathbf{u}_{\varepsilon} \times \nabla_{\boldsymbol{\tau}} \mathbf{u}_{\varepsilon}\right)\right|-C \varepsilon\right)^{2} .
$$

Step 3 . Thanks to Lemma 2 , there exists $\varepsilon_{*}$ such that the quantity $\alpha_{\varepsilon}$ defined in (73) satisfies

$$
\alpha_{\varepsilon} \geq 1-C \varepsilon>0 \quad \text { for any } 0<\varepsilon \leq \varepsilon_{*} .
$$

Fix $\varepsilon$ and $\rho$ such that $0<\varepsilon \leq \varepsilon_{*}$ and $\varepsilon<\rho \leq R_{*}$. By definition (34) of the $W_{\varepsilon}^{1,2}$-seminorm, there holds

$$
\frac{1}{2}\left|\mathbf{w}_{\varepsilon}\right|_{W_{\varepsilon}^{1,2}\left(\partial B_{\rho}\right)}^{2} \geq \frac{\alpha_{\varepsilon}(1-C \varepsilon)^{2}}{2} \int_{\partial B_{\rho}}\left|\nabla_{\mathrm{S}} \mathbf{w}_{\varepsilon}\right|^{2} \mathrm{~d} s .
$$

Set $m(\rho):=\min _{\partial B_{\rho}}\left|\mathbf{w}_{\varepsilon}\right|$ (note that $m(\rho) \in[1,1 / 2]$ ), and let $\boldsymbol{\tau}$ be a unit tangent field on $\partial B_{\rho}$. Using (78) and the definition (41) of $\jmath$, we obtain

$$
\frac{1}{2}\left|\mathbf{w}_{\varepsilon}\right|_{W_{\varepsilon}^{1,2}\left(\partial B_{\rho}\right)}^{2} \geq \frac{\alpha_{\varepsilon} m^{2}(\rho)}{2} \int_{\partial B_{\rho}}\left|\left\langle\jmath\left(\mathbf{u}_{\varepsilon}\right), \boldsymbol{\tau}\right\rangle-C \varepsilon\right|^{2} \mathrm{~d} s .
$$

By applying Jensen inequality, we deduce

$$
\begin{aligned}
\frac{1}{2}\left|\mathbf{w}_{\varepsilon}\right|_{W_{\varepsilon}^{1,2}\left(\partial B_{\rho}\right)}^{2} & \geq \frac{\alpha_{\varepsilon} m^{2}(\rho)}{2 \mathscr{H}^{1}\left(\partial B_{\rho}\right)}\left|\int_{\partial B_{\rho}} \jmath\left(\mathbf{u}_{\varepsilon}\right)-C \varepsilon \mathscr{H}^{1}\left(\partial B_{\rho}\right)\right|^{2} \\
& \stackrel{(77)}{\geq} \frac{\alpha_{\varepsilon} m^{2}(\rho)}{4 \pi \rho+C \rho^{2}}\left|\int_{\partial B_{\rho}} \jmath\left(\mathbf{u}_{\varepsilon}\right)-C \varepsilon \mathscr{H}^{1}\left(\partial B_{\rho}\right)\right|^{2} .
\end{aligned}
$$


Using Lemma 6, and arguing as in the proof of (57), we can evaluate the integral of $\jmath\left(\mathbf{u}_{\varepsilon}\right)$ in terms of $d:=\operatorname{ind}\left(\mathbf{u}_{\varepsilon}, B_{\rho}\right)$ and the Gauss curvature $G$ :

$$
\frac{1}{2}\left|\mathbf{w}_{\varepsilon}\right|_{W_{\varepsilon}^{1,2}\left(\partial B_{\rho}\right)}^{2} \geq \frac{\alpha_{\varepsilon} m^{2}(\rho)}{4 \pi \rho+C \rho^{2}}\left|2 \pi d-\int_{B_{\rho}} G-C \varepsilon \mathscr{H}^{1}\left(\partial B_{\rho}\right)\right|^{2} .
$$

Now, the Gauss curvature $G$ is bounded and $\varepsilon<\rho \leq R_{*}$, so the integral of $G$ is uniformly bounded by $C R_{*}^{2}$, while $\varepsilon \mathscr{H}^{1}\left(\partial B_{\rho}\right) \leq C R_{*}^{2}$. Thus, reducing the value of $R_{*}$ if necessary, we can assume that

$$
u:=\frac{1}{2 \pi}\left|\int_{B_{\rho}} G+C \varepsilon \mathscr{H}^{1}\left(\partial B_{\rho}\right)\right|<\frac{1}{2} .
$$

If $|d|>1$, then $|d-u|^{2} \geq d^{2}-2 u|d| \geq|d|$ and hence

$$
\frac{1}{2}\left|\mathbf{w}_{\varepsilon}\right|_{W_{\varepsilon}^{1,2}\left(\partial B_{\rho}\right)}^{2} \geq \frac{\alpha_{\varepsilon} \pi m^{2}(\rho)}{\rho+C \rho^{2}}|d| .
$$

An elementary computation, based on the fact that $u \leq C \rho^{2}$, shows that the same inequality is satisfied also if $|d|=1$, provided that we take a larger constant $C$ in the denominator, and we reduce again the value of $R_{*}$ if necessary. Finally, (81) is trivially satisfied if $d=0$.

Step 4. Suppose that $m(\rho)<1$, and let $x_{\rho} \in \partial B_{\rho}$ be such that $\left|\mathbf{w}_{\varepsilon}\left(x_{\rho}\right)\right|=$ $m(\rho)$. We have $\left|\mathbf{w}_{\varepsilon}\right| \leq(1+m(\rho)) / 2$ on $B_{\rho^{\prime}}\left(x_{\rho}\right)$, where

$$
\rho^{\prime}:=\frac{1-m(\rho)}{2 \operatorname{Lip}\left(\mathbf{w}_{\varepsilon}\right)} .
$$

Now, $\mathbf{w}_{\varepsilon}$ has Lipschitz constant $\operatorname{Lip}\left(\mathbf{w}_{\varepsilon}\right) \leq C \varepsilon^{-1}$, thanks to Lemma 3. Thus, since we have assumed that $\rho>\varepsilon$, we conclude that

$$
\mathscr{H}^{1}\left(\partial B_{\rho} \cap B_{\rho^{\prime}}\left(x_{\rho}\right)\right) \geq C \rho^{\prime} \geq C \varepsilon(1-m(\rho)) .
$$

Thus, by applying Lemma 5 , we estimate

$$
\frac{1}{2}\left|\mathbf{w}_{\varepsilon}\right|_{W_{\varepsilon}^{1,2}\left(\partial B_{\rho}\right)}^{2} \geq \frac{C}{\varepsilon^{2}} \int_{\partial B_{\rho} \cap B_{\rho^{\prime}}\left(x_{\rho}\right)}\left(1-\left|\mathbf{w}_{\varepsilon}\right|^{2}\right)^{2} \mathrm{~d} s \geq \frac{C}{\varepsilon}(1-m(\rho))^{3} .
$$

Note that (82) trivially holds when $m(\rho)=1$. Now, (75) follows by combining (79), (81) and (82).

Step 5 (Proof of (76)). The function $f: m \in[0,1] \mapsto A m^{2} \vee B(1-m)^{3}$ achieves its minimum value at the point $m_{0}$ such that $A m_{0}^{2}=B\left(1-m_{0}\right)^{3}$. From this equality, we deduce that

$$
\frac{B}{A}\left(1-m_{0}\right)^{3}=m_{0}^{2} \leq 1,
$$

whence $m_{0} \geq 1-(A / B)^{1 / 3}$ and $f\left(m_{0}\right) \geq A\left(1-2(A / B)^{1 / 3}\right)$. Substituting for $A$ and $B$ the expressions in (74), and using (73), yields (76). 
Following Jerrard [27], it will be useful to reformulate the lower bound (75) in terms of a function $\Lambda_{\varepsilon}$, defined by

$$
\Lambda_{\varepsilon}(r):=\int_{0}^{r} \lambda_{\varepsilon}(\rho, 1) \wedge \frac{C_{*}}{\varepsilon} \mathrm{d} \rho \quad \text { for } r>0 .
$$

We first collect a few properties of $\Lambda_{\varepsilon}$ (see also [27, Proposition 3.1]).

Lemma 10 The function $\Lambda_{\varepsilon}$ satisfies

$$
\Lambda_{\varepsilon}(r+s) \leq \Lambda_{\varepsilon}(r)+\Lambda_{\varepsilon}(s), \quad \Lambda_{\varepsilon}(r) \leq \Lambda_{\varepsilon}(s), \quad \frac{\Lambda_{\varepsilon}(r)}{r} \geq \frac{\Lambda_{\varepsilon}(s)}{s}
$$

for any $0<r \leq s$. Moreover, there holds

$$
\Lambda_{\varepsilon}(r) \geq(1-C \varepsilon) \pi \log \frac{r}{\varepsilon}-C
$$

for any $r \in\left(\varepsilon, R_{*}\right]$ (where $R_{*}$ is given by Lemma 9) and some $\varepsilon$-independent constant $C$.

Proof. It is clear by the definition (74) that $\lambda_{\varepsilon}$ is positive and decreasing; then (84) follows by elementary calculus. As for the lower bound (85), Equation (76) implies that

$$
\lambda_{\varepsilon}(\rho, 1) \wedge \frac{C}{\varepsilon} \geq \frac{(1-C \varepsilon) \pi}{\rho+C \rho^{2}}-C \varepsilon^{1 / 3} \rho^{-4 / 3}
$$

for any $\rho \in\left(c_{1} \varepsilon, R_{*}\right]$, for some constant $c_{1}>0$. By integrating both sides of this inequality with respect to $\rho \in\left(c_{1} \varepsilon, r\right)$, we deduce

$$
\begin{aligned}
\Lambda_{\varepsilon}(r) \geq & (1-C \varepsilon) \pi\left\{\log \frac{r}{c_{1} \varepsilon}+\log \frac{C \varepsilon+1}{C r+1}\right\}+C \varepsilon^{1 / 3}\left(R^{1 / 3}-r^{1 / 3}\right) \\
& \stackrel{\geq}{c_{1} \varepsilon<r<R_{*}}(1-C \varepsilon) \pi \log \frac{r}{\varepsilon}-C,
\end{aligned}
$$

where the constant $C$ in the right-hand side depends only on $c_{1}$ and $R_{*}$. If $c_{1} \leq 1$, then the lemma follows immediately. Otherwise, we note that, by choosing $C$ large enough, the right-hand side of (85) can be made non-positive for every $r \in\left[\varepsilon, c_{1} \varepsilon\right]$, so that (85) holds trivially.

We state a lower bound for the energy on annuli in terms of the function $\Lambda_{\varepsilon}$.

Lemma 11 For any $\varepsilon \in\left(0, \varepsilon_{*}\right]$, any $x_{0} \in M$, any $\varepsilon<r<R \leq R_{*}$ (where $\varepsilon_{*}, R_{*}$ are given by Lemma 9) and any $\mathbf{v}_{\varepsilon} \in \mathrm{T}_{\varepsilon}\left(\mathcal{T}_{\varepsilon} ; \mathbb{S}^{2}\right)$ such that $\left|\mathbf{w}_{\varepsilon}\right| \geq$ $1 / 2$ on $A_{r, R}:=B_{R}\left(x_{0}\right) \backslash B_{r}\left(x_{0}\right)$, there holds

$$
\frac{1}{2}\left|\mathbf{w}_{\varepsilon}\right|_{W_{\varepsilon}^{1,2}\left(A_{r, R}\right)}^{2} \geq|d|\left\{\Lambda_{\varepsilon}\left(\frac{R}{|d|}\right)-\Lambda_{\varepsilon}\left(\frac{r}{|d|}\right)\right\},
$$

where $d:=\operatorname{ind}\left(\mathbf{u}_{\varepsilon}, B_{R}\left(x_{0}\right)\right)$.

The proof of this lemma follows by integrating the lower bound (75); see [27, Proposition 3.2] for details. 


\subsection{The ball construction}

In this section, we recall the "ball construction" as presented by Jerrard [27] (a similar construction was independently introduced by Sandier [40], see also [43]). In contrast with [27], our lower bound (Lemma 11) is only valid for annuli with outer radius $\leq R_{*}$, so we need to make sure that this constraint is preserved by the construction.

Throughout this section, we fix a sequence of discrete fields $\mathbf{v}_{\varepsilon} \in \mathrm{T}\left(\mathcal{T}_{\varepsilon} ; \mathbb{S}^{2}\right)$, for $\varepsilon \in\left(0, \varepsilon_{*}\right]$, that satisfies the logarithmic energy bound $(\mathrm{H})$. We define the set $S_{\varepsilon}:=\left\{x \in M:\left|\mathbf{w}_{\varepsilon}(x)\right| \leq 1 / 2\right\}$ and the measure

$$
\nu_{\varepsilon}\left(\mathbf{v}_{\varepsilon}\right):=\frac{1}{2 \pi}\left(\mu_{\varepsilon}\left(\mathbf{v}_{\varepsilon}\right)+G \mathrm{~d} S\right)
$$

where $\mu_{\varepsilon}\left(\mathbf{v}_{\varepsilon}\right)$ is given by (56). Since $\left(\mathbf{v}_{\varepsilon}\right)$ is fixed, throughout this section we write $\nu_{\varepsilon}$ instead of $\nu_{\varepsilon}\left(\mathbf{v}_{\varepsilon}\right)$. If $E \subseteq M$ is a Borel set with $\partial E \subseteq M \backslash S_{\varepsilon}$, Equation (57) implies that $\nu_{\varepsilon}(E)=\operatorname{ind}\left(\mathbf{u}_{\varepsilon}, E\right)$. The sequence $\left(\nu_{\varepsilon}\right)$ is precompact in the flat topology if and only if $\left(\mu_{\varepsilon}\left(\mathbf{v}_{\varepsilon}\right)\right)$ is, and hence (by Proposition 2), if and only if $\left(\widehat{\mu}_{\varepsilon}\left(\mathbf{v}_{\varepsilon}\right)\right)$ is. We will also need the following notation: given a closed ball $B$, we denote by $\operatorname{rad}(B)$ its radius. If $\mathscr{B}$ is a finite collection of closed balls, we set spt $\mathscr{B}:=\cup_{B \in \mathscr{B}} B$.

Lemma 12 There exists an $\varepsilon$-independent constant $\beta$ such that, for any $T \in$ $\mathcal{T}_{\varepsilon}$,

$$
P(T) \cap S_{\varepsilon} \neq \emptyset \quad \text { implies } \quad \frac{1}{2}\left|\mathbf{w}_{\varepsilon}\right|_{W_{\varepsilon}^{1,2}(P(T))}^{2} \geq \beta .
$$

Proof. Suppose that there is a point $x_{0} \in P(T)$ such that $\left|\mathbf{w}_{\varepsilon}\left(x_{0}\right)\right| \leq 1 / 2$. Arguing as in the proof of Lemma 9, Step 4, we deduce that $\left|\mathbf{w}_{\varepsilon}\right| \leq 3 / 4$ on a ball $B_{\rho^{\prime}}\left(x_{0}\right)$ with $\rho^{\prime} \geq C \varepsilon$. Then, using also the assumptions $\left(\mathrm{H}_{1}\right)$ and $\left(\mathrm{H}_{2}\right)$, we see that $\mathscr{H}^{2}\left(P(T) \cap B_{\rho^{\prime}}\left(x_{0}\right)\right) \geq C \varepsilon$ and hence, by Lemma 5 , we estimate

$$
\frac{1}{2}\left|\mathbf{w}_{\varepsilon}\right|_{W_{\varepsilon}^{1,2}(P(T))}^{2} \geq \frac{C}{\varepsilon^{2}} \int_{P(T) \cap B_{\rho^{\prime}}\left(x_{\rho}\right)}\left(1-\left|\mathbf{w}_{\varepsilon}\right|^{2}\right)^{2} \mathrm{~d} S \geq C .
$$

Lemma 13 There exists an $\varepsilon$-independent constant $C$ such that, for any $T \in$ $\mathcal{T}_{\varepsilon}$, there holds $\left|\nu_{\varepsilon}(P(T))\right| \leq C$.

Proof. We have that

$$
\left|\nu_{\varepsilon}(P(T))\right| \stackrel{(86)}{\leq}\left|\mu_{\varepsilon}\left(\mathbf{v}_{\varepsilon}\right)[P(T)]\right|+C \varepsilon^{2} \stackrel{(56)}{=}\left|\int_{\partial(P(T))} \jmath\left(\mathbf{u}_{\varepsilon}\right)\right|+C \varepsilon^{2}
$$

(we have used that the Gauss curvature is bounded and the surface area of $P(T)$ is $\leq C \varepsilon^{2}$, which follows from $\left(\mathrm{H}_{1}\right)$ and $\left.\left(\mathrm{H}_{2}\right)\right)$. Using now the definition (55) of $\mathbf{u}_{\varepsilon}$, we compute that $\jmath\left(\mathbf{u}_{\varepsilon}\right)=\eta_{\varepsilon}\left(\left|\tilde{\mathbf{u}}_{\varepsilon}\right|\right) \jmath\left(\tilde{\mathbf{u}}_{\varepsilon}\right)$ and hence $\left|\jmath\left(\mathbf{u}_{\varepsilon}\right)\right| \leq$ $\operatorname{Lip}\left(\tilde{\mathbf{u}}_{\varepsilon}\right)$. Combining (55) with the Lipschitz bound (37) for $\mathbf{w}_{\varepsilon}$, we see that $\operatorname{Lip}\left(\tilde{\mathbf{u}}_{\varepsilon}\right) \leq \operatorname{Lip}\left(\mathbf{w}_{\varepsilon}\right) \leq C \varepsilon^{-1}$. Thus

$$
\left|\nu_{\varepsilon}(P(T))\right| \leq C \varepsilon^{-1} \mathscr{H}^{1}(\partial P(T))+C \varepsilon^{2} \leq C,
$$

where we have used that $\mathscr{H}^{1}(\partial P(T)) \leq C \varepsilon$, due to $\left(\mathrm{H}_{1}\right)$ and $\left(\mathrm{H}_{2}\right)$. 
For any $T \in \mathcal{T}_{\varepsilon}$ such that $P(T) \cap S_{\varepsilon} \neq \emptyset$, we consider the smallest closed ball $B$ of centre $P\left(x_{T}\right)$ such that $P(T) \subseteq B$. Let $\mathscr{B}_{\varepsilon}$ be the collection of such balls. Thanks to the assumption $\left(\mathrm{H}_{2}\right)$, any $B \in \mathscr{B}_{\varepsilon}$ satisfies

$$
C^{-1} \varepsilon \leq \operatorname{rad}(B) \leq C \varepsilon .
$$

Moreover, each ball $B \in \mathscr{B}_{\varepsilon}$ intersects $P(T)$ for at most $C$ triangles $T \in \mathcal{T}_{\varepsilon}$, where $C$ is an $\varepsilon$-independent constant. Therefore, from (87) and Lemma 13 we deduce that

$$
C^{-1} \varepsilon \leq s_{\varepsilon}:=\min _{B \in \mathscr{B}_{\varepsilon}} \frac{\operatorname{rad}(B)}{\left|\nu_{\varepsilon}(B)\right|} \leq C \varepsilon .
$$

(To prove the upper bound, note that $\operatorname{spt} \mathscr{B}_{\varepsilon} \supseteq \operatorname{spt}\left(\nu_{\varepsilon}\right)$ and that $\left|\nu_{\varepsilon}(B)\right| \geq 1$ as soon as $B \cap \operatorname{spt}\left(\nu_{\varepsilon}\right) \neq \emptyset$. Here, we are assuming WLOG that $\nu_{\varepsilon} \not \equiv 0$, otherwise $\left.s_{\varepsilon}=+\infty\right)$. Finally, as a consequence of Lemma 12 and the energy bound $(\mathrm{H})$, we obtain

$$
\#\left(\mathscr{B}_{\varepsilon}\right) \leq C|\log \varepsilon|
$$

The following proposition is adapted from [27, Proposition 4.1] (see also [42, Proposition 5.4])

Proposition 3. There exists an (E-independent) positive constant $C$ such that, for any $s \in\left[s_{\varepsilon}, C R_{*} \#\left(\mathscr{B}_{\varepsilon}\right)^{-1}\right]$, there exists a family of pairwise disjoint, closed balls $\mathscr{B}_{\varepsilon}(s)$ with the following properties.

(i) $\operatorname{spt} \mathscr{B}_{\varepsilon} \subseteq \operatorname{spt} \mathscr{B}_{\varepsilon}(s) \subseteq \operatorname{spt} \mathscr{B}_{\varepsilon}(t)$ for any $s_{\varepsilon} \leq s \leq t \leq C R_{*} \#\left(\mathscr{B}_{\varepsilon}\right)^{-1}$.

(ii) For any $B \in \mathscr{B}_{\varepsilon}(s)$, there holds

$$
\frac{1}{2}\left|\mathbf{w}_{\varepsilon}\right|_{W_{\varepsilon}^{1,2}\left(B \backslash \operatorname{spt} \mathscr{B}_{\varepsilon}\right)}^{2} \geq \frac{\operatorname{rad}(B)}{s}\left(\Lambda_{\varepsilon}(s)-\Lambda_{\varepsilon}\left(s_{\varepsilon}\right)\right) .
$$

(iii) For any $B \in \mathscr{B}_{\varepsilon}(s)$, there holds $\operatorname{rad}(B) \geq s\left|\nu_{\varepsilon}(B)\right|$.

(iv) There holds

$$
\sum_{B \in \mathscr{B}_{\varepsilon}(s)} \operatorname{rad}(B) \leq \frac{s}{s_{\varepsilon}} \sum_{B \in \mathscr{B}_{\varepsilon}} \operatorname{rad}(B) .
$$

Sketch of the proof. If the balls in $\mathscr{B}_{\varepsilon}$ are not pairwise disjoint, the construction starts with a merging phase, that is, we select a pair of balls $B, B^{\prime} \in \mathscr{B}_{\varepsilon}$ such that $B \cap B^{\prime} \neq \emptyset$ and we replace them with a new closed ball $B^{\text {new }}$ such that $B^{\text {new }} \supseteq B \cup B^{\prime}, \operatorname{rad}\left(B^{\text {new }}\right)=\operatorname{rad}(B)+\operatorname{rad}\left(B^{\prime}\right)$. We repeat this operation until we obtain a collection of pairwise disjoint closed balls, which we call $\mathscr{B}_{\varepsilon}^{\prime}$. If all the balls in the original collection $\mathscr{B}_{\varepsilon}$ were pairwise disjoint, then $\mathscr{B}_{\varepsilon}=\mathscr{B}_{\varepsilon}^{\prime}$. Set $\mathscr{B}_{\varepsilon}\left(s_{\varepsilon}\right):=\mathscr{B}_{\varepsilon}$, so (i), (iii), (iv) are trivially satisfied and (ii) is also satisfied, because of (88).

Now we perform an expansion phase, i.e. we let the parameter $s$ grow continuously, and we let the "minimizing balls" (i.e., the balls $B$ such that $\operatorname{rad}(B)=$ $\left.s\left|\nu_{\varepsilon}(B)\right|\right)$ grow, leaving the other unchanged. More precisely, if $\mathscr{B}_{\varepsilon}^{\prime}=\left\{B_{i}\right\}_{i=1}^{k}$ and $x_{i}$ is the centre of $B_{i}$, then the elements of $\mathscr{B}_{\varepsilon}(s)$ are defined by

$$
B_{i}(s):= \begin{cases}B_{i} & \text { if } \operatorname{rad}\left(B_{i}\right)>s\left|\nu_{\varepsilon}\left(B_{i}\right)\right| \\ \bar{B}_{i}\left(x_{i}, s\left|\nu_{\varepsilon}\left(B_{i}\right)\right|\right) & \text { otherwise. }\end{cases}
$$


For $s$ small enough, the balls $B_{i}(s)$ 's are pairwise disjoint. We also have $\left|\nu_{\varepsilon}\left(B_{i}\right)\right|=\left|\nu_{\varepsilon}\left(B_{i}(s)\right)\right|$, because $\left(B_{i}(s) \backslash B_{i}\right) \cap \operatorname{spt} \mathscr{B}_{\varepsilon}=\emptyset$ and $\operatorname{spt}\left(\nu_{\varepsilon}\right) \subseteq \operatorname{spt} \mathscr{B}_{\varepsilon}$. If for some $s^{*}$ there happens $B_{i}\left(s^{*}\right) \cap B_{j}\left(s^{*}\right) \neq \emptyset$ for $i \neq j$, then we stop the expansion phase. We define $\mathscr{B}_{\varepsilon}\left(s^{*}\right)$ as the family of balls obtained from $\left\{B_{i}\left(s^{*}\right)\right\}_{i=1}^{k}$ via merging. For $s>s^{*}$, we repeat an expansion phase according to the same rule as (90), until two or more balls touch and we perform a merging phase again, and so on.

Arguing as in [27, Proposition 4.1], one can show that $\mathscr{B}_{\varepsilon}(s)$ is a family of closed balls that satisfies (i), (ii) and (iii). (Actually, (ii) appears in a slightly different form, but the same argument applies.) The proof of (ii) relies on Lemma 11; in order to apply this lemma, we need to make sure that the radii of all the balls we consider are $\leq R_{*}$. However, if we temporarily assume that (iv) holds, then (using (87) and (88) as well) we see that

$$
\sum_{B \in \mathscr{B}_{\varepsilon}(s)} \operatorname{rad}(B) \leq C s \#\left(\mathscr{B}_{\varepsilon}\right) .
$$

Therefore, we have $\operatorname{rad}(B) \leq R_{*}$ for any $B \in \mathscr{B}_{\varepsilon}(s)$ and any $s \leq C^{-1} R_{*} \#\left(\mathscr{B}_{\varepsilon}\right)$.

To prove (iv), we note that the quantity $\sum_{B \in \mathscr{B}_{\varepsilon}(s)} \operatorname{rad}(B)$ is preserved during each merging phase. Then, for a fixed $s$, let $s_{1}<\ldots<s_{k}<s$ be the values of the parameter when merging occurred, and take $B_{i}(s) \in \mathscr{B}_{\varepsilon}(s)$. From (90), we see that

$$
\begin{aligned}
\operatorname{rad}\left(B_{i}(s)\right) & =\min \left\{\operatorname{rad}\left(B_{i}\left(s_{k}\right)\right), s\left|\nu_{\varepsilon}\left(B_{i}(s)\right)\right|\right\} \leq s\left|\nu_{\varepsilon}\left(B_{i}\left(s_{k}\right)\right)\right| \\
& \stackrel{(i i i)}{\leq} \frac{s}{s_{k}} \operatorname{rad}\left(B_{i}\left(s_{k}\right)\right)
\end{aligned}
$$

(we have used that $s \mapsto\left|\nu_{\varepsilon}\left(B_{i}(s)\right)\right|$ is constant during each expansion phase). Thus,

$$
\sum_{B \in \mathscr{B}_{\varepsilon}(s)} \operatorname{rad}(B) \leq \frac{s}{s_{k}} \sum_{B \in \mathscr{B}_{\varepsilon}\left(s_{k}\right)} \operatorname{rad}(B) .
$$

Now, we complete the proof of (iv) arguing by induction.

As an immediate consequence of Proposition 3, using (87), (88) the definition (83) of $\Lambda_{\varepsilon}$ and (85) in Lemma 10, we obtain

Corollary 1 For any $s \in\left[s_{\varepsilon}, C R_{*} \#\left(\mathscr{B}_{\varepsilon}\right)^{-1}\right]$, there exists a family of pairwise disjoint, closed balls $\mathscr{B}_{\varepsilon}(s)$ which satisfies the following properties:

(i) $\operatorname{spt} \mathscr{B}_{\varepsilon} \subseteq \operatorname{spt} \mathscr{B}_{\varepsilon}(s) \subseteq \operatorname{spt} \mathscr{B}_{\varepsilon}(t)$ for any $s_{\varepsilon} \leq s \leq t \leq R_{*} \#\left(\mathscr{B}_{\varepsilon}\right)^{-1}$;

(ii) for any $B \in \mathscr{B}_{\varepsilon}(s)$, there holds

$$
\frac{1}{2}\left|\mathbf{w}_{\varepsilon}\right|_{W_{\varepsilon}^{1,2}\left(B \backslash \operatorname{spt} \mathscr{B}_{\varepsilon}\right)}^{2} \geq\left|\nu_{\varepsilon}(B)\right|\left(\pi(1-C \varepsilon) \log \frac{s}{\varepsilon}-C\right)
$$

(iii) there holds $\sum_{B \in \mathscr{B}_{\varepsilon}(s)} \operatorname{rad}(B) \leq C s \#\left(\mathscr{B}_{\varepsilon}\right)$. 
5.3 Proof of the zero-order $\Gamma$-convergence

We state and prove a zero-order $\Gamma$-convergence result in terms of the measures $\nu_{\varepsilon}\left(\mathbf{v}_{\varepsilon}\right)$. Given a measure $\mu \in X$ with $\mu=\sum_{i} d_{i} \delta_{x_{i}}$, we set

$$
\sigma_{0}(\mu):=\frac{1}{2} \min \left\{\min _{j \neq i} \operatorname{dist}\left(x_{i}, x_{j}\right) \text {, injectivity radius of } M\right\} .
$$

Proposition 4. Suppose that the assumptions $\left(\mathrm{H}_{1}\right),\left(\mathrm{H}_{2}\right)$ and $\left(\mathrm{H}_{3}\right)$ are satisfied. Then, the following results hold.

(i) Compactness. If $\left(\mathbf{v}_{\varepsilon}\right)$ is a sequence in $\mathrm{T}\left(\mathcal{T}_{\varepsilon} ; \mathbb{S}^{2}\right)$ that satisfies the energy bound $(\mathrm{H})$ then, up to subsequences, $\nu_{\varepsilon}\left(\mathbf{v}_{\varepsilon}\right) \stackrel{\text { flat }}{\longrightarrow} \mu$ for some $\mu \in X$.

(ii) Localized $\Gamma$-liminf inequality. Let $\left(\mathbf{v}_{\varepsilon}\right)$ be a sequence in $\mathrm{T}\left(\mathcal{T}_{\varepsilon} ; \mathbb{S}^{2}\right)$ such that $\nu_{\varepsilon}\left(\mathbf{v}_{\varepsilon}\right) \stackrel{\text { flat }}{\longrightarrow} \mu$ for some $\mu \in X, \mu=\sum_{i=1}^{K} d_{i} \delta_{x_{i}}$. Then, there exists a constant $C$ such that, for any $i \in\{1, \ldots, K\}$ and any $0<\sigma \leq \sigma_{0}(\mu)$, there holds

$$
\liminf _{\varepsilon \rightarrow 0}\left(\frac{1}{2}\left|\mathbf{w}_{\varepsilon}\right|_{W_{\varepsilon}^{1,2}\left(B_{\sigma}\left(x_{i}\right)\right)}^{2}-\pi\left|d_{i}\right| \log \frac{\sigma}{\varepsilon}\right) \geq C .
$$

(iii) $\Gamma$-limsup inequality. For any $\mu \in X$ there exists a sequence $\left(\mathbf{v}_{\varepsilon}\right)$ in $\mathrm{T}\left(\mathcal{T}_{\varepsilon} ; \mathbb{S}^{2}\right)$ such that $\nu_{\varepsilon}\left(\mathbf{v}_{\varepsilon}\right) \stackrel{\text { flat }}{\longrightarrow} \mu$ and

$$
\limsup _{\varepsilon \rightarrow 0} \frac{\left|\mathbf{w}_{\varepsilon}\right|_{W_{\varepsilon}^{1,2}(M)}^{2}}{2|\log \varepsilon|} \leq \pi|\mu|(M) .
$$

The proof of this Proposition is adapted from [2, Theorem 3.1]. Throughout the proof, we write $\nu_{\varepsilon}$ instead of $\nu_{\varepsilon}\left(\mathbf{v}_{\varepsilon}\right)$ when no confusion is possible.

Proof of (i) - Compactness. Let $\mathscr{B}_{\varepsilon}^{1}:=\mathscr{B}_{\varepsilon}\left(s_{\varepsilon}^{1}\right)$ be the family of balls given by Corollary 1 for the choice of parameter $s_{\varepsilon}^{1}:=\varepsilon^{1 / 2}$. If $\varepsilon$ is small enough, we have

$$
s_{\varepsilon} \stackrel{(88)}{\leq} \varepsilon^{1 / 2} \leq \frac{C R_{*}}{|\log \varepsilon|} \stackrel{(89)}{\leq} \frac{C R_{*}}{\#\left(\mathscr{B}_{\varepsilon}\right)},
$$

so $s_{\varepsilon}^{1}$ satisfies the assumptions of Corollary 1. By (i) in Corollary 1, we have that $\operatorname{spt}\left(\nu_{\varepsilon}\right) \subseteq \operatorname{spt} \mathscr{B}_{\varepsilon} \subseteq \operatorname{spt} \mathscr{B}_{\varepsilon}^{1}$, while (ii) implies

$$
\frac{1}{2}\left|\mathbf{w}_{\varepsilon}\right|_{W_{\varepsilon}^{1,2}(B)}^{2} \geq\left|\nu_{\varepsilon}(B)\right|\left(\frac{\pi}{2}(1-C \varepsilon)|\log \varepsilon|-C\right)
$$

for any $B \in \mathscr{B}_{\varepsilon}^{1}$. Summing up this inequality over all the $B$ 's, using Lemma 3 and the energy bound $(\mathrm{H})$, one sees that

$$
\sum_{B \in \mathscr{B} 1}\left|\nu_{\varepsilon}(B)\right| \leq C
$$


for an $\varepsilon$-independent constant $C$. We introduce the measures

$$
\nu_{\varepsilon}^{1}:=\sum_{B \in \mathscr{B}_{\varepsilon}^{1}} \nu_{\varepsilon}(B) \delta_{x(B)},
$$

where $x(B)$ denotes the centre of the ball $B$ and we note that belong to $X$. In fact, we have

$$
\nu_{\varepsilon}^{1}(M)=\sum_{B \in \mathscr{B}_{\varepsilon}^{1}} \nu_{\varepsilon}(B)=\sum_{B \in \mathscr{B}_{\varepsilon}^{1}} \operatorname{ind}\left(\mathbf{v}_{\varepsilon}, B\right)=\operatorname{ind}\left(\mathbf{v}_{\varepsilon}, M\right)=\chi(M) .
$$

The measures $\nu_{\varepsilon}^{1}$ have uniformly bounded mass and therefore flat-converge to an element of $X$, up to extraction of a subsequence. On the other hand, (iii) in Corollary 1 implies

$$
\sum_{B \in \mathscr{B}_{\varepsilon}^{1}} \operatorname{rad}(B) \leq C \varepsilon^{1 / 2} \#\left(\mathscr{B}_{\varepsilon}\right) \stackrel{(89)}{\leq} C \varepsilon^{1 / 2}|\log \varepsilon| .
$$

Then, arguing as in [2, Theorem 3.1.(i)], one can show that $\left\|\nu_{\varepsilon}-\nu_{\varepsilon}^{1}\right\|_{\text {flat }} \rightarrow 0$, which yields compactness for the sequence $\left(\nu_{\varepsilon}\right)$. (see also [3, Theorem 3.3] for more details).

Proof of (ii) $-\Gamma$-liminf. Fix $i \in\{1, \ldots, K\}$ and $0<\sigma \leq \sigma_{0}(\mu)$. By extraction of a non-relabelled subsequence, we can assume WLOG that

$$
\lim _{\varepsilon \rightarrow 0}\left(\frac{1}{2}\left|\mathbf{w}_{\varepsilon}\right|_{W_{\varepsilon}^{1,2}\left(B_{\sigma}\left(x_{i}\right)\right)}^{2}-\pi\left|d_{i}\right||\log \varepsilon|\right)<+\infty
$$

(and, in particular, the limit exists). Arguing as in [2, Theorem 3.1.(ii)], we see that $\left\|\nu_{\varepsilon}\left(\mathbf{v}_{\varepsilon}\right)-\nu_{\varepsilon}\left(\overline{\mathbf{v}}_{\varepsilon}\right)\right\|_{\text {flat }} \rightarrow 0$, where $\overline{\mathbf{v}}_{\varepsilon}$ denotes the restriction of $\mathbf{v}_{\varepsilon}$ to $B_{\sigma}\left(x_{i}\right)$, and that $\nu_{\varepsilon}\left(\overline{\mathbf{v}}_{\varepsilon}\right)$ flat-converges to $d_{i} \delta_{x_{i}}$. Therefore, we can repeat the ball construction of Section 5.2 with $M$ replaced by $B_{\sigma}\left(x_{i}\right)$ and $\mathbf{v}_{\varepsilon}$ replaced by $\overline{\mathbf{v}}_{\varepsilon}$. (This guarantees that no ball "coming from outside" enters $B_{\sigma}\left(x_{i}\right)$.) We still write $\nu_{\varepsilon}$ instead of $\nu_{\varepsilon}\left(\overline{\mathbf{v}}_{\varepsilon}\right)$.

For a fixed $\gamma \in(0,1)$, we apply Corollary 1 with $s=s_{\varepsilon}^{2}:=\varepsilon^{\gamma}$. (One can check, arguing as in the proof of (i), that the assumptions of Corollary 1 are satisfied.) The collection of balls $\mathscr{B}_{\varepsilon}\left(s_{\varepsilon}^{2}\right) \operatorname{satisfies} \operatorname{spt}\left(\nu_{\varepsilon}\right) \subseteq \operatorname{spt} \mathscr{B}_{\varepsilon}\left(s_{\varepsilon}^{2}\right)$,

$$
\sum_{B \in \mathscr{B}_{\varepsilon}\left(s_{\varepsilon}^{2}\right)} \operatorname{rad}(B) \leq C \varepsilon^{\gamma} \#\left(\mathscr{B}_{\varepsilon}\right) \stackrel{(89)}{\leq} C \varepsilon^{\gamma}|\log \varepsilon|
$$

and

$$
\frac{1}{2}\left|\mathbf{w}_{\varepsilon}\right|_{W_{\varepsilon}^{1,2}(B)}^{2} \geq\left|\nu_{\varepsilon}(B)\right|(\pi(1-\gamma)(1-C \varepsilon)|\log \varepsilon|-C)
$$

for any $B \in \mathscr{B}_{\varepsilon}\left(s_{\varepsilon}^{2}\right) \backslash \mathscr{B}_{\varepsilon}$. Let $\mathscr{B}_{\varepsilon}^{2}:=\left\{B \in \mathscr{B}_{\varepsilon}\left(s_{\varepsilon}^{2}\right): B \subseteq B_{\sigma}\left(x_{i}\right)\right\}$ and $\nu_{\varepsilon}^{2}:=$ $\sum_{B \in \mathscr{B}_{\varepsilon}^{2}} \nu_{\varepsilon}(B) \delta_{x(B)}$. Arguing again as in [2], we see that $\left\|\nu_{\varepsilon}^{2}-\nu_{\varepsilon}\right\|_{\text {flat }} \rightarrow 0$, 
so $\nu_{\varepsilon}^{2}$ flat-converges to $d_{i} \delta_{x_{i}}$ and, in particular, $\liminf _{\varepsilon \rightarrow 0}\left|\nu_{\varepsilon}^{2}\right|\left(B_{\sigma}\left(x_{i}\right)\right) \geq\left|d_{i}\right|$. Now, by summing up the inequality (92) with respect to $B \in \mathscr{B}_{\varepsilon}^{2}$, we deduce

$$
\frac{1}{2}\left|\mathbf{w}_{\varepsilon}\right|_{W_{\varepsilon}^{1,2}\left(B_{\sigma}\left(x_{i}\right)\right)}^{2} \geq \pi(1-\gamma)(1-C \varepsilon)\left|\nu_{\varepsilon}^{2}\right|\left(B_{\sigma}\left(x_{i}\right)\right)|\log \varepsilon|-C .
$$

If $\liminf _{\varepsilon \rightarrow 0}\left|\nu_{\varepsilon}^{2}\right|\left(B_{\sigma}\left(x_{i}\right)\right)>\left|d_{i}\right|$, then in fact $\liminf _{\varepsilon \rightarrow 0}\left|\nu_{\varepsilon}^{2}\right|\left(B_{\sigma}\left(x_{i}\right)\right) \geq\left|d_{i}\right|+1$ (because $\nu_{\varepsilon}^{2}$ is integer-valued) and hence the $\Gamma$-liminf inequality (ii) follows, provided that we choose $\gamma$ such that $(1-\gamma)\left(\left|d_{i}\right|+1\right)>\left|d_{i}\right|$. Otherwise, we have that $\left|\nu_{\varepsilon}^{2}\right|\left(B_{\sigma}\left(x_{i}\right)\right)=\left|d_{i}\right|$ for $\varepsilon$ small enough. Then, we can write

$$
\nu_{\varepsilon}^{2}=\sum_{j=1}^{k} p_{j}^{\varepsilon} \delta_{y_{i}^{\varepsilon}}
$$

where the numbers $p_{j}^{\varepsilon} \in \mathbb{Z}$ all have the same sign and satisfy $\sum_{j} p_{j}^{\varepsilon}=d_{i}$, and $y_{j}^{\varepsilon} \rightarrow x_{i}$ as $\varepsilon \rightarrow 0$. By taking $\varepsilon$ small enough, we can assume that $y_{j}^{\varepsilon} \in$ $B_{\sigma / 2}\left(x_{i}\right)$ for all $j$.

We fix a positive number $\eta>0$, and we apply Corollary 1 with $s=$ $s_{\varepsilon}^{3}:=\eta \#\left(\mathscr{B}_{\varepsilon}\right)^{-1}$. (We choose $\eta$ small enough that $s_{\varepsilon}^{3} \leq C R_{*} \#\left(\mathscr{B}_{\varepsilon}\right)^{-1}$, so the assumptions of Corollary (1) are satisfied.) We find a collection of balls $\mathscr{B}_{\varepsilon}^{3}:=$ $\mathscr{B}_{\varepsilon}\left(s_{\varepsilon}^{3}\right)$ that satisfies $\operatorname{spt}\left(\nu_{\varepsilon}^{2}\right) \subseteq \operatorname{spt} \mathscr{B}_{\varepsilon}^{3}$,

$$
\begin{gathered}
\sum_{B \in \mathscr{B}_{\varepsilon}^{3}} \operatorname{rad}(B) \leq C \eta \\
\frac{1}{2}\left|\mathbf{w}_{\varepsilon}\right|_{W_{\varepsilon}^{1,2}\left(B \backslash \operatorname{spt} \mathscr{B}_{\varepsilon}\right)}^{2} \geq\left|\nu_{\varepsilon}(B)\right|\left(\pi(1-C \varepsilon) \log \frac{\eta}{\varepsilon \#\left(\mathscr{B}_{\varepsilon}\right)}-C\right)
\end{gathered}
$$

Thanks to (93) and the fact that $\operatorname{spt}\left(\nu_{\varepsilon}^{2}\right) \subseteq \operatorname{spt} \mathscr{B}_{\varepsilon}^{3}$, dist $\left(\operatorname{spt} \nu_{\varepsilon}^{2}, \partial B_{\sigma}\left(x_{i}\right)\right) \geq$ $\sigma / 2$, we can choose $\eta$ so small that $B \subseteq B_{\sigma}\left(x_{i}\right)$ for any $B \in \mathscr{B}_{\varepsilon}^{3}$. Then, using also the fact that all the $p_{j}^{\varepsilon}$ have the same sign and sum up to $d_{i}$, we see that $\sum_{B \in \mathscr{B}_{\varepsilon}^{3}}\left|\nu_{\varepsilon}^{2}(B)\right|=\left|d_{i}\right|$ and hence, by (94),

$$
\frac{1}{2}\left|\mathbf{w}_{\varepsilon}\right|_{W_{\varepsilon}^{1,2}\left(B_{\sigma}\left(x_{i}\right) \backslash \operatorname{spt} \mathscr{B}_{\varepsilon}\right)}^{2} \geq \pi\left|d_{i}\right|(1-C \varepsilon) \log \frac{\eta}{\varepsilon \#\left(\mathscr{B}_{\varepsilon}\right)}-C .
$$

On the other hand, Lemma 12 implies that

$$
\frac{1}{2}\left|\mathbf{w}_{\varepsilon}\right|_{W_{\varepsilon}^{1,2}\left(\operatorname{spt} \mathscr{B}_{\varepsilon}\right)}^{2} \geq \sum_{T \in \mathcal{T}_{\varepsilon}: P(T) \cap S_{\varepsilon} \neq \emptyset} \frac{1}{2}\left|\mathbf{w}_{\varepsilon}\right|_{W_{\varepsilon}^{1,2}(P(T))}^{2} \geq \beta \#\left(\mathscr{B}_{\varepsilon}\right) .
$$

Thus, we have

$$
\begin{aligned}
\frac{1}{2}\left|\mathbf{w}_{\varepsilon}\right|_{W_{\varepsilon}^{1,2}\left(B_{\sigma}\left(x_{i}\right)\right)}^{2} & \geq \pi\left|d_{i}\right|(1-C \varepsilon)|\log \varepsilon| \\
& \quad-\pi\left|d_{i}\right|(1-C \varepsilon) \log \frac{\#\left(\mathscr{B}_{\varepsilon}\right)}{\eta}+\beta \#\left(\mathscr{B}_{\varepsilon}\right)-C \\
& \geq \pi\left|d_{i}\right||\log \varepsilon|-C
\end{aligned}
$$


Proof of (iii) $-\Gamma$-limsup. Fix $\mu=\sum_{i=1}^{K} d_{i} \delta_{x_{i}} \in X$, and suppose that $d_{i} \neq$ 0 for any $i$. By a diagonal argument, it suffices to show the following: for any $\delta$ and any countable subsequence of $\varepsilon \searrow 0$, there exists a (non-relabelled) subsequence such that

$$
\limsup _{\varepsilon \rightarrow 0} \frac{\left|\mathbf{w}_{\varepsilon}\right|_{W_{\varepsilon}^{1,2}(M)}^{2}}{2|\log \varepsilon|} \leq \pi|\mu|(M)+\delta .
$$

Let us fix $\delta>0$ and the countable subsequence of $\varepsilon$. We also fix a small parameter $0<\sigma \leq \sigma_{0}(\mu)$. For $i \in\{1, \ldots, K\}$ and $j \in\left\{1, \ldots,\left|d_{i}\right|\right\}$, we let $z_{i, j}^{\varepsilon}:=\varepsilon^{\sigma} \exp \left(2 \pi \iota j\left|d_{i}\right|^{-1}\right) \in \mathbb{C}$ (where $\iota$ is the imaginary unit). By taking $\varepsilon$ small enough, we can assume that $\left|z_{i, j}^{\varepsilon}\right|<\sigma$. We define a map $\tilde{\mathbf{u}}_{i}^{\varepsilon}: B_{\sigma} \subseteq \mathbb{C} \rightarrow \mathbb{C}$ by

$$
\tilde{\mathbf{u}}_{i}^{\varepsilon}(z):=\prod_{j=1}^{d_{i}} \frac{z-z_{i, j}^{\varepsilon}}{\left|z-z_{i, j}^{\varepsilon}\right|} \quad \text { if } d_{i}>0, \quad \tilde{\mathbf{u}}_{i}^{\varepsilon}(z):=\prod_{j=1}^{-d_{i}} \frac{\overline{z-z_{i, j}^{\varepsilon}}}{\left|z-z_{i, j}^{\varepsilon}\right|} \quad \text { otherwise. }
$$

Using normal coordinates $\varphi_{i}: B_{\sigma} \subseteq \mathbb{C} \rightarrow M$ such that $\varphi_{i}(0)=x_{i}$, we can transport $\tilde{\mathbf{u}}_{i}^{\varepsilon}$ to a vector field on $B_{\sigma}\left(x_{i}\right) \subseteq M$, i.e. we define $\mathbf{u}_{i}^{\varepsilon}\left(\varphi_{i}(z)\right):=$ $\left\langle\mathrm{d} \varphi_{i}(z), \tilde{\mathbf{u}}_{i}^{\varepsilon}(z)\right\rangle$ for $z \in B_{\sigma} \subseteq \mathbb{C}$. Since $\sum_{i} \operatorname{ind}\left(\mathbf{u}_{i}^{\varepsilon}, B_{\sigma}\left(x_{i}\right)\right)=\sum_{i} d_{i}=\chi(N)$, we find a smooth vector field $\mathbf{u}$ on $M_{\sigma}:=M \backslash \cup_{i} B_{\sigma}\left(x_{i}\right)$ that satisfies $\mathbf{u}=\mathbf{u}_{i}^{\varepsilon}$ on $\partial B_{\sigma}\left(x_{i}\right)$ for each $i$. We define $\mathbf{u}^{\varepsilon}:=\mathbf{u}_{i}^{\varepsilon}$ on $B_{\sigma}\left(x_{i}\right)$ and $\mathbf{u}^{\varepsilon}:=\mathbf{u}$ on $M_{\sigma}$. The tangent field $\mathbf{u}^{\varepsilon} \in W^{1,1}\left(M, \mathbb{R}^{3}\right)$ is smooth except at the points $x_{i, j}^{\varepsilon}:=$ $\varphi_{i}\left(z_{i, j}^{\varepsilon}\right) \rightarrow x_{i}$ and hence, by Lemma 6 ,

$$
\frac{1}{2 \pi}\left(\star \mathrm{d} \jmath\left(\mathbf{u}^{\varepsilon}\right)-G\right)=\sum_{i, j} \operatorname{sign}\left(d_{i}\right) \delta_{x_{i, j}^{\varepsilon}} \stackrel{\text { flat }}{\longrightarrow} \sum_{i} d_{i} \delta_{x_{i}}=\mu \quad \text { as } \varepsilon \rightarrow 0 .
$$

Let $\mathbf{v}_{\varepsilon}$ be the discrete field defined by $\mathbf{v}_{\varepsilon}(i):=\mathbf{u}^{\varepsilon}(i)$ for $i \in \mathcal{T}_{\varepsilon}^{0}$, and let $\mathbf{w}_{\varepsilon}:=\widehat{P}_{\varepsilon}^{-1} \circ \widehat{\mathbf{v}}_{\varepsilon}$. We have

$$
\left|\nabla_{\mathrm{s}} \mathbf{w}_{\varepsilon}\right| \stackrel{\left(\mathrm{H}_{1}\right)}{\leq} C\left|\nabla_{\varepsilon} \widehat{\mathbf{v}}_{\varepsilon}\right| \leq C\left|\nabla_{\mathrm{s}} \mathbf{u}^{\varepsilon}\right|
$$

where the last inequality follows by basic properties of the affine interpolant. Setting $D_{i}^{\varepsilon}:=B_{\sigma}\left(x_{i}\right) \backslash \cup_{j} B_{\varepsilon}\left(x_{i, j}^{\varepsilon}\right)$, and using (34) and Lemma 2, we obtain

$$
\begin{aligned}
\frac{1}{2}\left|\mathbf{w}_{\varepsilon}\right|_{W_{\varepsilon}^{1,2}(M)}^{2}= & \frac{1}{2} \sum_{i=1}^{K}\left|\mathbf{w}_{\varepsilon}\right|_{W_{\varepsilon}^{1,2}\left(D_{i}^{\varepsilon}\right)}^{2}+\frac{1}{2} \sum_{i, j}\left|\mathbf{w}_{\varepsilon}\right|_{W_{\varepsilon}^{1,2}\left(B_{\varepsilon}\left(x_{i, j}^{\varepsilon}\right)\right)}^{2} \\
& +\frac{1}{2}\left|\mathbf{w}_{\varepsilon}\right|_{W_{\varepsilon}^{1,2}\left(M_{\sigma}\right)}^{2} \\
\leq & \frac{1+C \varepsilon}{2} \sum_{i=1}^{K} \int_{D_{i}^{\varepsilon}}\left|\nabla_{\mathrm{s}} \mathbf{u}^{\varepsilon}\right|^{2} \mathrm{~d} S+\sum_{i=1}^{K} \operatorname{Lip}\left(\mathbf{w}_{\varepsilon}\right)^{2} \mathscr{H}^{2}\left(B_{\varepsilon}\left(x_{i}\right)\right) \\
& \quad+\frac{1+C \varepsilon}{2} \int_{M_{\sigma}}\left|\nabla_{\mathrm{s}} \mathbf{u}\right|^{2} \mathrm{~d} S \\
& (\stackrel{37}{\leq}) \frac{1+C \varepsilon}{2} \sum_{i=1}^{K} \int_{D_{i}^{\varepsilon}}\left|\nabla_{\mathrm{s}} \mathbf{u}^{\varepsilon}\right|^{2} \mathrm{~d} S+C_{\sigma},
\end{aligned}
$$


where $C_{\sigma}$ is a positive constant, depending on $\sigma$. The integral of $\left|\nabla_{\mathrm{s}} \mathbf{v}\right|^{2}$ on each $D_{i}^{\varepsilon}$ can be evaluated using (96) and the fact that $\operatorname{Lip}\left(\varphi_{i \mid B_{\sigma}\left(x_{i}\right)}\right) \leq 1+C \sigma$ :

$$
\frac{1+C \varepsilon}{2} \int_{D_{i}^{\varepsilon}}\left|\nabla_{\mathrm{s}} \mathbf{u}^{\varepsilon}\right|^{2} \mathrm{~d} S \leq\left(\pi(1+C \sigma)\left|d_{i}\right|+C \sigma\left|d_{i}\right|^{2}\right)|\log \varepsilon|+C_{\sigma},
$$

whence

$$
\limsup _{\varepsilon \rightarrow 0} \frac{\left|\mathbf{w}_{\varepsilon}\right|_{W_{\varepsilon}^{1,2}(M)}^{2}}{2|\log \varepsilon|} \leq \pi(1+C \sigma)|\mu|(M)+C \sigma(|\mu|(M))^{2}
$$

and, choosing $\sigma$ so small that $C \sigma|\mu|(M)+C|\mu|(M)^{2} \leq \delta$, (95) follows.

To conclude the proof, we only need to show that $\nu_{\varepsilon}\left(\mathbf{v}_{\varepsilon}\right)$ flat-converges to $\mu$. Using $\left(\mathrm{H}_{2}\right)$, the definition of affine interpolant, and the fact that

$$
\left|\nabla_{\mathrm{s}} \mathbf{u}^{\varepsilon}(x)\right| \leq \frac{C_{\sigma}}{\operatorname{dist}\left(x,\left\{x_{i, j}^{\varepsilon}\right\}\right)} \quad \text { for } x \in B_{\sigma}\left(x_{i}\right)
$$

(as a consequence of (96)), one finds positive numbers $\lambda, \varepsilon_{1}$ such that, for any $0<\varepsilon \leq \varepsilon_{1}$, there holds $\left|\mathbf{w}_{\varepsilon}\right| \geq 1 / 2$ on $A_{\varepsilon}:=M \backslash \cup_{i, j} B_{\lambda \varepsilon}\left(x_{i, j}^{\varepsilon}\right)$. Thanks to Lemma 4, this implies $\left|\mathbf{u}_{\varepsilon}\right| \geq 1 / 4$ if $\varepsilon$ is small enough, where $\mathbf{u}_{\varepsilon}$ is the field defined by (55). Then, using (57) and (86), we obtain that $\nu_{\varepsilon}\left(\mathbf{v}_{\varepsilon}\right)[B]=0$ if $B \subseteq A_{\varepsilon}$. On the other hand, we also have $\mu[B]=0$ if $B \subseteq A_{\varepsilon}$, due to (97). Thus, for any Lipschitz function $\varphi$ on $M \operatorname{such}$ that $\sup |\varphi|+\operatorname{Lip}(\varphi) \leq 1$, there holds

$$
\begin{aligned}
\left\langle\nu_{\varepsilon}\left(\mathbf{v}_{\varepsilon}\right)-\mu, \varphi\right\rangle & =\sum_{i, j} \int_{B_{\lambda \varepsilon}\left(x_{i, j}^{\varepsilon}\right)} \varphi \mathrm{d}\left(\nu_{\varepsilon}\left(\mathbf{v}_{\varepsilon}\right)-\mu\right) \\
& =\sum_{i, j} \int_{B_{\lambda \varepsilon}\left(x_{i, j}^{\varepsilon}\right)}\left(\varphi-\varphi\left(x_{i}\right)\right) \mathrm{d}\left(\nu_{\varepsilon}\left(\mathbf{v}_{\varepsilon}\right)-\mu\right) \\
& \leq C \lambda \varepsilon\left(\left|\nu_{\varepsilon}\left(\mathbf{v}_{\varepsilon}\right)\right|+|\mu|\right)(M),
\end{aligned}
$$

and Lemma 13 implies

$$
\left|\nu_{\varepsilon}\left(\mathbf{v}_{\varepsilon}\right)\right|(M) \leq C \#\left\{T \in \mathcal{T}_{\varepsilon}: P(T) \backslash A_{\varepsilon} \neq \emptyset\right\} \stackrel{\left(\mathrm{H}_{2}\right)}{\leq} C .
$$

Combining (98) and (99), we conclude that $\left\|\nu\left(\mathbf{v}_{\varepsilon}\right)-\mu\right\|_{\text {flat }} \leq C \lambda \varepsilon$.

\section{The first-order $\Gamma$-convergence: location of defects and their energetics}

6.1 The renormalized energy

In this Subsection we resume the concept of Renormalized energy that we have introduced in (18) and we state its main properties. We recall that we have set

$$
\mathbb{W}_{\text {intr }}(\mathbf{v}):=\lim _{\delta \rightarrow 0}\left(\frac{1}{2} \int_{M_{\delta}}|\mathrm{D} \mathbf{v}|^{2} \mathrm{~d} S-\mathscr{K} \pi|\log \delta|\right) \quad \text { for } \mathbf{v} \in \mathcal{V}_{\mathscr{K}}
$$


where for $\mathscr{K} \in \mathbb{N}$ we have set (see (17))

$$
\begin{aligned}
\mathcal{V}_{\mathscr{K}}:= & \left\{\mathbf{v} \in L^{2}\left(M ; \mathbb{S}^{2}\right): \text { there exist }\left(x_{i}\right)_{i=1}^{\mathscr{K}} \in M^{\mathscr{K}}\right. \text { s.t. } \\
& \left.\mathbf{v} \in W_{\text {tan,loc }}^{1,2}\left(M \backslash \cup_{i=1}^{\mathscr{K}} x_{i} ; \mathbb{S}^{2}\right) \text { and }\left|\operatorname{ind}\left(\mathbf{v}, x_{i}\right)\right|=1 \text { for any } i\right\} .
\end{aligned}
$$

The object in (100) is well defined for the following reasons.

First of all, a standard construction based on the Poincaré-Hopf theorem (see, e.g., [18, Proposition 1.5]) shows that the set $\mathcal{V}_{\mathscr{K}}$ is non empty if and only if $\mathscr{K} \geq|\chi(M)|$ and $\mathscr{K} \equiv \chi(M) \bmod 2$, that is, $\mathscr{K}$ is even. Second, for any $\mathbf{v} \in \mathcal{V}_{\mathscr{K}}$ and any $\delta>0$ there holds that

$$
\frac{1}{2} \int_{M_{\delta}}|\mathrm{Dv}|^{2} \mathrm{~d} S-\mathscr{K} \pi|\log \delta|<+\infty .
$$

Third, for any $\mathbf{v} \in \mathcal{V}_{\mathscr{K}}$, there holds

$$
\begin{aligned}
\frac{\mathrm{d}}{\mathrm{d} \delta}\left(\frac{1}{2} \int_{M_{\delta}}|\mathrm{Dv}|^{2} \mathrm{~d} S-\mathscr{K} \pi|\log \delta|\right) & =\sum_{i=1}^{\mathscr{K}}\left(-\frac{1}{2} \int_{\partial B_{\delta}\left(x_{i}\right)}|\mathrm{Dv}|^{2} \mathrm{~d} S+\frac{\pi}{\delta}\right) \\
& \leq \sum_{i=1}^{\mathscr{K}}\left(-\frac{\left(2 \pi+C \delta^{2}\right)^{2}}{4 \pi \delta+C \delta^{2}}+\frac{\pi}{\delta}\right),
\end{aligned}
$$

where the last inequality follows by Lemma 21 below. Since the righ-hand side is bounded from above as $\delta \searrow 0$, we deduce that the limit in (100) exists and belongs to $(-\infty,+\infty]$.

Similar to the euclidean flat case (see [2]) we have the following dyadic decomposition of the intrinsic Renormalized Energy.

Lemma 14 Fix $\rho>$ small enough in such a way that $B_{\rho}\left(x_{i}\right)$ are pairwise disjoint for $i=1, \ldots, \mathscr{K}$. Then, for any $\mathbf{v} \in \mathcal{V}_{\mathscr{K}}$ there holds

$$
\begin{aligned}
\mathbb{W}_{\text {intr }}(\mathbf{v})= & \frac{1}{2} \int_{M \backslash \bigcup_{i=1}^{\mathscr{K}} B_{\rho}\left(x_{i}\right)}|\mathrm{Dv}|^{2} \mathrm{~d} S-\pi \mathscr{K}|\log \rho| \\
& +\sum_{i=1}^{\mathscr{K}} \sum_{j=0}^{+\infty}\left(\frac{1}{2} \int_{B_{2^{-j} \rho}\left(x_{i}\right) \backslash B_{2-(j+1)}\left(x_{i}\right)}|\mathrm{Dv}|^{2} \mathrm{~d} S-\pi \log 2\right) .
\end{aligned}
$$

Proof. For $l \in \mathbb{N}$ and some fixed $\rho>0$ as in the statement, we consider $\delta=\rho 2^{-(l+1)}$. Note that the limit $\delta \rightarrow 0$ corresponds to the limit $l \rightarrow+\infty$. Moreover, we dyadically decompose $B_{\rho}\left(x_{i}\right) \backslash B_{\rho 2-(l+1)}$ for any $i=1, \ldots, \mathscr{K}$. We thus obtain

$$
M \backslash \bigcup_{i=1}^{\mathscr{K}} B_{\delta}\left(x_{i}\right)=\left(M \backslash \bigcup_{i=1}^{\mathscr{K}} B_{\rho}\left(x_{i}\right)\right) \cup \bigcup_{i=1}^{\mathscr{K}} \bigcup_{j=0}^{l}\left(B_{2^{-j} \rho}\left(x_{i}\right) \backslash B_{2^{-(j+1)} \rho}\left(x_{i}\right)\right) .
$$


Thus, we have

$$
\begin{aligned}
& \int_{M \backslash \bigcup_{i=1}^{\mathscr{K}} B_{\delta}\left(x_{i}\right)}|\mathrm{D} \mathbf{v}|^{2} \mathrm{~d} S-\pi \mathscr{K}|\log \delta| \\
& =\int_{M \backslash \bigcup_{i=1}^{\mathscr{K}} B_{\rho}\left(x_{i}\right)}|\mathrm{D} \mathbf{v}|^{2} \mathrm{~d} S-\pi \mathscr{K}|\log \rho| \\
& \quad+\sum_{i=1}^{\mathscr{K}} \sum_{j=0}^{l} \int_{B_{2^{-j}}\left(x_{i}\right) \backslash B_{2-(j+1) \rho}\left(x_{i}\right)}|\mathrm{Dv}|^{2} \mathrm{~d} S-\pi \mathscr{K}(l+1) \log 2 \\
& =\int_{M \backslash \bigcup_{i=1}^{\mathscr{K}} B_{\rho}\left(x_{i}\right)}|\mathrm{D} \mathbf{v}|^{2} \mathrm{~d} S-\pi \mathscr{K}|\log \rho| \\
& \quad+\sum_{i=1}^{\mathscr{K}} \sum_{j=0}^{l}\left(\int_{B_{2-j_{\rho}}\left(x_{i}\right) \backslash B_{2-(j+1)}\left(x_{i}\right)}|\mathrm{D} \mathbf{v}|^{2} \mathrm{~d} S-\pi \log 2\right) .
\end{aligned}
$$

Therefore, by sending $l \rightarrow+\infty$ (i.e. $\delta \rightarrow 0$ ), the lemma follows.

Thanks to Lemma 14, the extrinsinc Renormalized Energy defined by (20) satisfies

$$
\begin{aligned}
\mathbb{W}(\mathbf{v})=\frac{1}{2} \int_{M \backslash \bigcup_{i=1}^{\mathscr{K}} B_{\rho}\left(x_{i}\right)}|\mathrm{D} \mathbf{v}|^{2} \mathrm{~d} S-\pi \mathscr{K}|\log \rho|+\frac{1}{2} \int_{M}|\mathrm{~d} \boldsymbol{\gamma}[\mathbf{v}]|^{2} \mathrm{~d} S \\
+\sum_{i=1}^{\mathscr{K}} \sum_{j=0}^{+\infty}\left(\frac{1}{2} \int_{B_{2^{-j} \rho}\left(x_{i}\right) \backslash B_{2-(j+1)_{\rho}\left(x_{i}\right)}}|\mathrm{D} \mathbf{v}|^{2} \mathrm{~d} S-\pi \log 2\right) .
\end{aligned}
$$

An interesting consequence of the above representation is that, for $\mathbf{v} \in \mathcal{V}_{\mathscr{K}}$ such that $\mathbb{W}(\mathbf{v})<+\infty$ there holds that, analogously to the euclidean case (see $[2$, Remark 4.4]),

$$
\begin{aligned}
& \lim _{j \rightarrow \infty} \frac{1}{2} \int_{B_{2-j}\left(x_{i}\right) \backslash B_{2-(j+1)_{\rho}}\left(x_{i}\right)}|\mathrm{Dv}|^{2} \mathrm{~d} S \\
& \quad=\lim _{j \rightarrow \infty} \frac{1}{2} \int_{B_{2-j_{\rho}}\left(x_{i}\right) \backslash B_{2-(j+1)_{\rho}}\left(x_{i}\right)} e(\mathbf{v}) \mathrm{d} S=\pi \log 2 .
\end{aligned}
$$

Consequently, we have

Lemma 15 The effective domain of $\mathbb{W}$ in $\mathcal{V}_{\mathscr{K}}$ is included in $W_{\tan }^{1,1}\left(M ; \mathbb{S}^{2}\right)$, namely

$$
\left\{\mathbf{v} \in \mathcal{V}_{\mathscr{K}}: \mathbb{W}(\mathbf{v})<+\infty\right\} \subseteq W_{\text {tan }}^{1,1}\left(M ; \mathbb{S}^{2}\right) \text {. }
$$

Proof. It is clearly sufficient to show that any $\mathbf{v} \in \mathcal{V}_{\mathscr{K}}$ with $\mathbb{W}(\mathbf{v})<+\infty$ is in $W^{1,1}\left(B_{\rho}\left(x_{i}\right)\right)(\rho>0$ smaller than the injectivity radius) for any $i=1, \ldots, \mathscr{K}$. We set $A_{j}^{i}:=B_{2^{-j} \rho}\left(x_{i}\right) \backslash B_{2^{-(j+1)} \rho}\left(x_{i}\right)$. There holds

$$
\int_{A_{j}^{i}}|\mathrm{D} \mathbf{v}| \mathrm{d} S \leq\left(\mathscr{H}^{2}\left(A_{j}^{i}\right)\right)^{1 / 2}\left(\int_{A_{j}^{i}}|\mathrm{D} \mathbf{v}|^{2} \mathrm{~d} S\right)^{1 / 2}
$$


and thus, thanks to (102)

$$
\int_{A_{j}^{i}}|\mathrm{Dv}| \mathrm{d} S \leq C \rho 2^{-j}, \quad \text { for all } i=1, \ldots, \mathscr{K} .
$$

Consequently, if we dyadically decompose $B_{\rho}\left(x_{i}\right)$ for any $i$ we get

$$
\int_{B_{\rho}\left(x_{i}\right)}|\mathrm{Dv}| \mathrm{d} S \leq C \sum_{j=1}^{+\infty} 2^{-j}<+\infty,
$$

which clearly gives the result.

6.2 The core energy

In this subsection we rigorously define the concept of core energy and discuss some of its properties. This object was introduced by Bethuel, Brexis and Hélein [12] and later extended to the discrete setting by Alicandro et al. [2].

Given a point $\bar{x} \in M$ and radii $\delta_{1}, \delta_{2}$ such that $\delta_{1}<\delta_{2}$, we denote by $A_{\delta_{1}, \delta_{2}}(\bar{x})$ the geodesic annulus

$$
A_{\delta_{1}, \delta_{2}}(\bar{x}):=B_{\delta_{2}}(\bar{x}) \backslash B_{\delta_{2}}(\bar{x}) \subseteq M .
$$

Let us fix a positive number $\delta$, smaller than the injectivity radius of $M$. We consider the minimization problem

$$
\begin{aligned}
\eta(\delta, \bar{x}):=\min _{\mathbf{w} \in W_{\text {tan }}^{1,2}\left(A_{\delta / 2, \delta}(\bar{x}) ; \mathbb{S}^{2}\right)}\left\{\frac{1}{2} \int_{A_{\delta / 2, \delta}(\bar{x})}|\mathrm{D} \mathbf{w}|^{2}+\right. & |\mathrm{d} \boldsymbol{\gamma}[\mathbf{w}]|^{2} \mathrm{~d} S: \\
& \operatorname{ind}(\mathbf{w}, \bar{x})=1\} .
\end{aligned}
$$

We denote with $\mathcal{H}(\delta, \bar{x})$ the set of its minimizers. $\mathcal{H}(\delta, \bar{x})$ is non-empty, as follows by standard arguments in the Calculus of Variations. We fix a minimizer $\mathbf{g}_{\delta} \in \mathcal{H}(\delta, \bar{x})$ for Problem (103) and, for $\varepsilon>0, \delta>0$, we set

$$
\gamma_{\bar{x}}(\varepsilon, \delta):=\min _{\mathbf{v} \in \mathrm{T}\left(\mathcal{T}_{\varepsilon}, S^{2}\right)}\left\{\frac{1}{2} \int_{\widehat{B_{\delta}(\bar{x})_{\varepsilon}}}\left|\nabla_{\varepsilon} \widehat{\mathbf{v}}\right|^{2} \mathrm{~d} S: \quad \mathbf{v}=\mathbf{g}_{\delta} \text { on } \partial_{\varepsilon} B_{\delta}(\bar{x})\right\} .
$$

Since $\bar{x}$ is fixed throughout this section, we will write $\gamma(\varepsilon, \delta)$ instead of $\gamma_{\bar{x}}(\varepsilon, \delta)$. We recall that $\widehat{B_{\delta}(\bar{x})_{\varepsilon}}$ is the union of the triangles $T \in \mathcal{T}_{\varepsilon}$ such that $P(T) \subseteq$ $B_{\delta}(\bar{x}), \partial_{\varepsilon} B_{\delta}(\bar{x}):=\partial\left(\widehat{B_{\delta}(\bar{x})_{\varepsilon}}\right) \cap \mathcal{T}_{\varepsilon}^{0}$, and $\widehat{\mathbf{v}}$ is the affine interpolant of the discrete field v. $\gamma(\varepsilon, \delta)$ depends on the choice of the point $\bar{x}$, of $\mathbf{g}_{\delta} \in \mathcal{H}(\delta, \bar{x})$ and of the triangulation $\mathcal{T}_{\varepsilon}$, even though we have dropped this dependence in the notation. We are interested in the asymptotic behaviour of $\gamma(\varepsilon, \delta)$ as $\varepsilon \searrow 0$, $\delta \searrow 0$. 
Proposition 5. Suppose that the sequence $\left(\mathcal{T}_{\varepsilon}\right)$ satisfies $\left(\mathrm{H}_{1}\right),\left(\mathrm{H}_{2}\right) .\left(\mathrm{H}_{3}\right)$ and $\left(\mathrm{H}_{4}\right)$. Then for any $\bar{x} \in M$ the following limits are finite and coincide:

$$
\gamma(\bar{x}):=\lim _{\delta \rightarrow 0} \liminf _{\varepsilon \rightarrow 0}\left(\gamma(\varepsilon, \delta)-\pi \log \frac{\delta}{\varepsilon}\right)=\lim _{\delta \rightarrow 0} \limsup _{\varepsilon \rightarrow 0}\left(\gamma(\varepsilon, \delta)-\pi \log \frac{\delta}{\varepsilon}\right) .
$$

It will be clear from the proof that the number $\gamma(\bar{x})$ depends on $\bar{x}$ and on the sequence $\left(\mathcal{T}_{\varepsilon}\right)$, but not on the choice of $\mathbf{g}_{\delta} \in \mathcal{H}(\delta, \bar{x})$. To prove Proposition 5 , we compare $\gamma(\varepsilon, \delta)$ with the solution of an auxiliary problem, defined as the "flat" counterpart of Problem (104). We know that the solution of the latter converges to a finite limit as $\varepsilon \searrow 0$, thanks to the analysis in [12] and [2], and hence we will be able to prove convergence for $\gamma(\varepsilon, \delta)$.

Before moving to the proof of Proposition 5, let us fix some notation. Let $\delta>0$ be smaller than the injectivity radius of $M$. Let $\bar{A}_{\delta / 2, \delta} \subseteq \mathbb{R}^{2}$ be the Euclidean annulus, centred at the origin, with radii $\delta / 2$ and $\delta$. The geodesic coordinates $\varphi: B_{\delta} \subseteq \mathbb{R}^{2} \rightarrow M$ induce a bijection $W^{1,2}\left(\bar{A}_{\delta / 2, \delta} ; \mathbb{S}^{1}\right) \rightarrow$ $W_{\tan }^{1,2}\left(A_{\delta / 2, \delta}(\bar{x}) ; \mathbb{S}^{2}\right):$ the push-forward $\varphi_{*} \overline{\mathbf{w}}$ of a field $\overline{\mathbf{w}} \in W^{1,2}\left(\bar{A}_{\delta / 2, \delta} ; \mathbb{S}^{1}\right)$ is defined by

$$
\left(\varphi_{*} \overline{\mathbf{w}}\right)(\varphi(x)):=\frac{\langle\mathrm{d} \varphi(x), \overline{\mathbf{w}}(x)\rangle}{|\langle\mathrm{d} \varphi(x), \overline{\mathbf{w}}(x)\rangle|}, \quad \text { for } x \in \bar{A}_{\delta / 2, \delta} .
$$

The pull-back of a field $\mathbf{w} \in W_{\tan }^{1,2}\left(A_{\delta / 2, \delta}(\bar{x}) ; \mathbb{S}^{2}\right)$ is given by $\varphi^{*} \mathbf{w}:=\left(\varphi^{-1}\right)_{*} \mathbf{w}$. A straightforward computation, based on the fact that $\mathrm{d} \varphi(0)=\operatorname{Id}_{\mathrm{T}_{\bar{x}} M}$, shows that

$$
E_{\operatorname{extr}}\left(\varphi_{*} \overline{\mathbf{w}} ; A_{\delta / 2, \delta}(\bar{x})\right) \leq\left(\frac{1}{2}+\mathrm{O}(\delta)\right) \int_{\bar{A}_{\delta / 2, \delta}}|\nabla \overline{\mathbf{w}}|^{2} \mathrm{~d} S
$$

as $\delta \rightarrow 0$ (see (14) for the definition of the extrinsic energy $E_{\text {extr }}$ ); in a similar way, there holds

$$
\frac{1}{2} \int_{\bar{A}_{\delta / 2, \delta}}\left|\nabla\left(\varphi^{*} \mathbf{w}\right)\right|^{2} \mathrm{~d} S \leq(1+\mathrm{O}(\delta)) E_{\text {extr }}\left(\mathbf{w} ; A_{\delta / 2, \delta}(\bar{x})\right) .
$$

Moreover, we have ind $(\mathbf{w}, \bar{x})=\operatorname{ind}\left(\varphi^{*} \mathbf{w}, 0\right)$. The push-forward of discrete fields $\varphi_{*}: \mathrm{T}\left(\overline{\mathcal{T}}_{\varepsilon}, \mathbb{S}^{2}\right) \rightarrow \mathrm{T}\left(\mathcal{T}_{\varepsilon}, \mathbb{S}^{1}\right)$, along with its inverse $\varphi^{*}$, is defined in a similar way.

Using $\varphi$, we can compare minimizers $\mathbf{g}_{\delta} \in \mathcal{H}(\delta, \bar{x})$ of Problem (103) with minimizers of the corresponding Euclidean problem, namely

$$
\min _{\overline{\mathbf{w}} \in W^{1,2}\left(\bar{A}_{\delta / 2, \delta} ; \mathbb{S}^{1}\right)}\left\{\frac{1}{2} \int_{\bar{A}_{\delta / 2, \delta}}|\nabla \overline{\mathbf{w}}|^{2} \mathrm{~d} S, \quad \text { ind }(\overline{\mathbf{w}}, 0)=1\right\} .
$$

The minimizers of (108) are exactly the fields of the form $\overline{\mathbf{h}}_{R}(x):=R x /|x|$, where $R \in \mathrm{SO}(2)$ is a constant rotation matrix.

Lemma 16 For any $\delta>0$ (smaller than the injectivity radius of $M$ ) and any $\mathbf{g}_{\delta} \in \mathcal{H}(\delta, \bar{x})$, there exist $R=R\left(\delta, \mathbf{g}_{\delta}\right) \in \mathrm{SO}(2)$ such that

$$
\lim _{\delta \searrow 0}\left\|\varphi^{*} \mathbf{g}_{\delta}-\overline{\mathbf{h}}_{R}\right\|_{W^{1,2}\left(A_{\delta / 2, \delta}(\bar{x})\right)}=0 .
$$


Proof. Set $\overline{\mathbf{g}}_{\delta}:=\varphi^{*} \mathbf{g}_{\delta}$ and $\mathbf{h}_{R}:=\varphi_{*} \overline{\mathbf{h}}_{R}$. By minimality of $\mathbf{g}_{\delta}$ and (106), we have

$$
\begin{aligned}
E_{\text {extr }}\left(\mathbf{g}_{\delta}, A_{\delta / 2, \delta}(\bar{x})\right) & \leq E_{\text {extr }}\left(\mathbf{h}_{R}, A_{\delta / 2, \delta}(\bar{x})\right) \\
& \stackrel{(106)}{\leq}\left(\frac{1}{2}+\mathrm{O}(\delta)\right) \int_{\bar{A}_{\delta / 2, \delta}}\left|\nabla \overline{\mathbf{h}}_{R}\right|^{2} \mathrm{~d} S=\pi \log 2+\mathrm{O}(\delta) .
\end{aligned}
$$

Then, using (107), we obtain

$$
\frac{1}{2} \int_{\bar{A}_{\delta / 2, \delta}}\left|\nabla \overline{\mathbf{g}}_{\delta}\right|^{2} \mathrm{~d} S \leq(1+\mathrm{O}(\delta)) E_{\text {extr }}\left(\mathbf{g}_{\delta}, A_{\delta / 2, \delta}(\bar{x})\right) \leq \pi \log 2+\mathrm{O}(\delta) .
$$

Let $\overline{\mathbf{f}}_{\delta}: A_{1 / 2,1} \rightarrow \mathbb{S}^{1}$ be defined by $\overline{\mathbf{f}}_{\delta}(x):=\overline{\mathbf{g}}_{\delta}(\delta x)$. From the previous inequality, it follows that

$$
\lim _{\delta \searrow 0} \frac{1}{2} \int_{\bar{A}_{1 / 2,1}}\left|\nabla \overline{\mathbf{f}}_{\delta}\right|^{2} \mathrm{~d} S=\lim _{\delta \searrow 0} \frac{1}{2} \int_{\bar{A}_{\delta / 2, \delta}}\left|\nabla \overline{\mathbf{g}}_{\delta}\right|^{2} \mathrm{~d} S=\pi \log 2,
$$

where the right-hand side is exactly the minimum value for Problem (108). Thus, $\overline{\mathbf{f}}_{\delta}$ is a minimizing sequence for Problem (108) and, by standard arguments in the Calculus of Variations, we find a subsequence that converges strongly in $W^{1,2}\left(\bar{A}_{1 / 2,1}\right)$ to a minimizer of (108). Now, arguing by contradiction, we deduce that

$$
\lim _{\delta \searrow 0} \inf _{R \in \mathrm{SO}(2)}\left\|\overline{\mathbf{f}}_{\delta}-\overline{\mathbf{h}}_{R}\right\|_{W^{1,2}\left(A_{1 / 2,1}\right)}=0,
$$

whence the lemma follows.

We point out a couple of immediate, but useful, consequences of Lemma 16.

Lemma 17 For any $\delta>0$ (smaller than the injectivity radius of $M$ ) and any $\mathbf{g}_{\delta} \in \mathcal{H}(\delta, \bar{x})$, there exist $R=R\left(\delta, \mathbf{g}_{\delta}\right) \in \mathrm{SO}(2)$ such that

$$
\lim _{\delta \searrow 0} \sup _{t \in[3 \delta / 4, \delta]}\left\|\varphi^{*} \mathbf{g}_{\delta}-\overline{\mathbf{h}}_{R}\right\|_{W^{1 / 2,2}\left(\partial B_{t}\right)}=0 .
$$

Proof. By a scaling argument, we find a constant $C$ such that the norm of the trace operator $T_{\delta, t}: W^{1,2}\left(\bar{A}_{\delta / 2, \delta}\right) \rightarrow W^{1 / 2,2}\left(\partial B_{t}\right)$ is bounded by $C$, for any $t$ and $\delta$ satisfying $3 / 4 \leq t / \delta \leq 1$. Then, the lemma immediately follows from Lemma 16 and the continuity of $T_{t, \delta}$.

Lemma 18 We have that $\eta(\delta, \bar{x}) \rightarrow \pi \log 2$ as $\delta \searrow 0$, uniformly in $\bar{x} \in M$.

Proof. This follows from the arguments in the proof of Lemma 16. Note that, since $M$ is compact and smooth, the quantities $\mathrm{O}(\delta)$ that appear in (106)-(107) are bounded uniformly with respect to $\bar{x}$. 
Lemma 16 above remains valid with a similar proof also for vector fields $\mathbf{v} \in \mathcal{V}_{\mathscr{K}}$ with $\mathscr{K} \equiv \chi(M) \bmod 2$. The elements in $\mathcal{V}_{\mathscr{K}}$ are not necessarily minimizers of (103) but they satisfy, thanks to the dyadic decomposition of the renormalized energy (see (101) and (102)),

$$
\lim _{\delta \rightarrow 0} \frac{1}{2} \int_{A_{\delta, \delta / 2}\left(x_{i}\right)}|\mathrm{D} \mathbf{v}|^{2} \mathrm{~d} S=\lim _{\delta \rightarrow 0} \frac{1}{2} \int_{A_{\delta, \delta / 2}\left(x_{i}\right)}\left|\nabla_{\mathrm{s}} \mathbf{v}\right|^{2} \mathrm{~d} S=\pi \log 2
$$

for any $i=1, \ldots, \mathscr{K}$. The above convergence replaces (109) and thus we have

Lemma 19 Let $\mathscr{K} \equiv \chi(M) \bmod 2$ and consider $\mathbf{v} \in \mathcal{V}_{\mathscr{K}}$. Then, for any $\delta>$ 0 (smaller than the injectivity radius of $M$ ) and any $i=1, \ldots, \mathscr{K}$ there exist $R=R(\delta, i, \mathbf{g}) \in \mathrm{SO}(2)$ such that

$$
\lim _{\delta \searrow 0}\left\|\nabla_{\mathbf{s}} \mathbf{v}-\nabla_{\mathbf{s}} \mathbf{h}_{R}\right\|_{L^{2}\left(A_{\delta / 2, \delta}\left(x_{i}\right)\right)}=0 .
$$

The following lemma will also be useful in the proof of Proposition 5.

Lemma 20 Let $T \subseteq \mathbb{R}^{3}$ be a triangle of vertices $i_{0}, i_{1}, i_{2}$, and let $\mathbf{w}: T \rightarrow \mathbb{R}^{3}$ be an affine map such that $\left|\mathbf{w}\left(i_{k}\right)\right|=1$ for $k \in\{0,1,2\}$. Let $\phi$ be a diffeomorphism defined in a neighbourhood of $T$, and suppose that $\|\mathrm{d} \phi-\operatorname{Id}\|_{L^{\infty}} \leq \delta$ where $0<\delta \leq 1 / 2$. Let $S$ be the triangle of vertices $\phi\left(i_{0}\right), \phi\left(i_{1}\right), \phi\left(i_{2}\right)$, and let $\mathbf{z}$ be the unique affine map $S \rightarrow \mathbb{R}^{3}$ such that

$$
\mathbf{z}\left(\phi\left(i_{k}\right)\right)=\frac{\left\langle\mathrm{d} \phi\left(i_{k}\right), \mathbf{w}\left(i_{k}\right)\right\rangle}{\left|\left\langle\mathrm{d} \phi\left(i_{k}\right), \mathbf{w}\left(i_{k}\right)\right\rangle\right|} \quad \text { for } k \in\{0,1,2\} .
$$

Then, there holds

$$
\int_{S}|\nabla \mathbf{z}|^{2} \mathrm{~d} S \leq(1+C \delta) \int_{T}|\nabla \mathbf{w}|^{2} \mathrm{~d} S
$$

Proof. Thanks to the assumptions that $|\mathbf{w}|=1$ on the vertices of $T$ and $\| \mathrm{d} \phi-$ Id $\|_{L^{\infty}} \leq \delta \leq 1 / 2$, we see that $\mathbf{z}$ is well-defined and

$$
\begin{gathered}
\left|\mathbf{z}\left(i_{k}\right)-\mathbf{z}\left(i_{h}\right)\right| \leq(1+C \delta)\left|\mathbf{w}\left(i_{k}\right)-\mathbf{w}\left(i_{h}\right)\right| \\
(1-C \delta)\left|i_{k}-i_{h}\right| \leq\left|\phi\left(i_{k}\right)-\phi\left(i_{h}\right)\right| \leq(1+C \delta)\left|i_{k}-i_{h}\right|
\end{gathered}
$$

for any $k, h \in\{0,1,2\}$. Then, the lemma follows by a straighforward computation.

Proof of Proposition 5. For the sake of convenience, we split the proof into steps.

Step 1. Recall from Section (3.1) that $\overline{\mathcal{T}}_{\varepsilon}$ is the pull back of $\mathcal{T}_{\varepsilon}$ via $\varphi$, namely, $\overline{\mathcal{T}}_{\varepsilon}$ is a triangulation on $B_{\delta} \subseteq \mathbb{R}^{2}$ with set of vertices $\varphi^{-1}\left(B_{\delta}(\bar{x}) \cap \mathcal{T}_{\varepsilon}^{0}\right)$; three vertices in $\overline{\mathcal{T}}_{\varepsilon}$ span a triangle in $\overline{\mathcal{T}}_{\varepsilon}$ if and only if their images via $\varphi$ do. We consider the minimization problem

$$
\gamma_{1}(\varepsilon, \delta):=\min _{\mathbf{v} \in \mathrm{T}\left(\overline{\mathcal{T}}_{\varepsilon}, S^{1}\right)}\left\{\frac{1}{2} \int_{\widehat{\left(B_{\delta}\right)_{\varepsilon}}}\left|\nabla_{\varepsilon} \widehat{\mathbf{v}}\right|^{2} \mathrm{~d} S: \quad \mathbf{v}=\overline{\mathbf{g}}_{\delta} \text { on } \partial_{\varepsilon} B_{\delta}\right\},
$$


where $\overline{\mathbf{g}}_{\delta}:=\varphi^{*} \mathbf{g}_{\delta}$. We wish to show that

$$
(1-C \delta) \gamma_{1}(\varepsilon, \delta) \leq \gamma(\varepsilon, \delta) \leq(1+C \delta) \gamma_{1}(\varepsilon, \delta)
$$

Let $\mathbf{v} \in \mathrm{T}\left(\overline{\mathcal{T}}_{\varepsilon} ; \mathbb{S}^{2}\right)$ with $\mathbf{v}=\overline{\mathbf{g}}_{\delta}$ on $\partial_{\varepsilon} B_{\delta}$ be a competitor for Problem (110). The pull-back $\varphi_{*} \mathbf{v} \in \mathrm{T}\left(\mathcal{T}_{\varepsilon} ; \mathbb{S}^{2}\right)$ satisfies $\varphi_{*} \mathbf{v}=\mathbf{g}_{\delta}$ on $\partial_{\varepsilon} B_{\delta}(\bar{x})$, so $\varphi_{*} \mathbf{v}$ is an admissible competitor for Problem (104). By noting that $\mathrm{d} \varphi(0)=\operatorname{Id}_{\mathrm{T}_{x} M}$, and applying Lemma 20 on each triangle of $\overline{\mathcal{T}}_{\varepsilon}$, we obtain that

$$
\gamma(\varepsilon, \delta) \leq \frac{1}{2} \int_{\widehat{B_{\delta}(\bar{x})_{\varepsilon}}}\left|\nabla_{\varepsilon}\left(\widehat{\varphi_{*} \mathbf{v}}\right)\right|^{2} \mathrm{~d} S \leq\left(\frac{1}{2}+C \delta\right) \int_{\widehat{\left(B_{\delta}\right)_{\varepsilon}}}|\nabla \widehat{\mathbf{v}}|^{2} \mathrm{~d} S
$$

(In order to apply Lemma 20, we extend $\varphi$ to a 3 -dimensional diffeormorphism $\phi$ by setting $\phi\left(x_{1}, x_{2}, x_{3}\right):=\varphi\left(x_{1}, x_{2}\right)+x_{3}(\gamma \circ \varphi)\left(x_{1}, x_{2}\right)$ for $\left(x_{1}, x_{2}\right) \in$ $B_{\delta}$ and $x_{3}$ small enough.) Thus, by arbitrarity of $\mathbf{v}$, we deduce

$$
\gamma(\varepsilon, \delta) \leq(1+C \delta) \gamma_{1}(\varepsilon, \delta) .
$$

A similar argument gives the other inequality in (111).

Step 2. Following the notation in Section 3.1, we define a triangulation on $B_{\delta / \varepsilon} \subseteq \mathbb{R}^{2}$ by setting

$$
\mathcal{S}_{\varepsilon}:=\left\{\frac{1}{\varepsilon} T: T \in \overline{\mathcal{T}}_{\varepsilon}\right\} .
$$

Thanks to $\left(\mathrm{H}_{2}\right)$, there exists an $\varepsilon$-independent constant $\Lambda$ such that, for any $\varepsilon$ and any $T \in \mathcal{S}_{\varepsilon}$, the affine bijection $\phi_{T}$ from the reference triangle $T_{\text {ref }} \subseteq \mathbb{R}^{2}$ spanned by $(0,0),(1,0),(0,1)$ to $T$ satisfies

$$
\max \left\{\operatorname{Lip}\left(\phi_{T}\right), \operatorname{Lip}\left(\phi_{T}^{-1}\right)\right\} \leq \Lambda .
$$

By scaling, we deduce from (110) that

$$
\gamma_{1}(\varepsilon, \delta)=\min _{\mathbf{v} \in \mathrm{T}\left(\mathcal{S}_{\varepsilon}, \mathbb{S}^{1}\right)}\left\{\frac{1}{2} \int_{\widehat{\left(B_{\delta / \varepsilon}\right)_{\varepsilon}}}|\nabla \widehat{\mathbf{v}}|^{2} \mathrm{~d} S: \quad \mathbf{v}=\overline{\mathbf{g}}_{\varepsilon, \delta} \text { on } \partial_{\varepsilon} B_{\delta / \varepsilon}\right\},
$$

where $\widehat{\left(B_{\delta / \varepsilon}\right)_{\varepsilon}}$ is the union of the triangles $T \in \mathcal{S}_{\varepsilon}$ such that $T \subseteq B_{\delta / \varepsilon}$, $\partial_{\varepsilon} B_{\delta / \varepsilon}:=\partial \widehat{\left(B_{\delta / \varepsilon}\right)} \cap \mathcal{S}_{\varepsilon}^{0}$ and $\overline{\mathbf{g}}_{\varepsilon, \delta}: \bar{A}_{\delta /(2 \varepsilon), \delta / \varepsilon} \rightarrow \mathbb{R}^{2}$ is given by $\overline{\mathbf{g}}_{\varepsilon, \delta}(x):=$ $\overline{\mathbf{g}}_{\delta}(\varepsilon x)$. Let us define

$$
\gamma_{2}(\varepsilon, \delta)=\min _{\mathbf{v} \in \mathrm{T}\left(\mathcal{S}_{\varepsilon}, \mathbb{S}^{1}\right)}\left\{\frac{1}{2} \int_{\widehat{\left(B_{\delta / \varepsilon}\right)_{\varepsilon}}}|\nabla \widehat{\mathbf{v}}|^{2} \mathrm{~d} S: \quad \mathbf{v}=\overline{\mathbf{h}} \text { on } \partial_{\varepsilon} B_{\delta / \varepsilon}\right\},
$$

where $\overline{\mathbf{h}}(x)=\overline{\mathbf{h}}_{\mathrm{Id}}(x):=x /|x|$ is, modulo rotations, the unique minimizer of (108). We claim that there exists postive numbers $\sigma(\delta), r(\delta)$, depending only on $\delta$, such that

$$
\gamma_{2}(\varepsilon, \delta+\sigma(\delta) \delta)-r(\delta) \leq \gamma_{1}(\varepsilon, \delta) \leq \gamma_{2}(\varepsilon, \delta-\sigma(\delta) \delta)+r(\delta),
$$

and $\sigma(\delta) \rightarrow 0, r(\delta) \rightarrow 0$ as $\delta \searrow 0$. 
Thanks to (112), there exists a constant $\lambda_{0}$ (which does not depend on $\delta, \varepsilon$ ) such that $\partial_{\varepsilon} B_{\delta / \varepsilon} \subseteq \bar{B}_{\delta / \varepsilon} \backslash B_{\delta / \varepsilon-\lambda_{0}}$. Let $\sigma \in(0,1 / 2)$ be a parameter, possibly depending on $\delta$ but not on $\varepsilon$, to be chosen later. By taking $\varepsilon$ small enough, we can assume without loss of generality that $(1-\sigma / 2) \delta / \varepsilon \leq \delta / \varepsilon-\lambda_{0}$, so that

$$
\partial_{\varepsilon} B_{\delta / \varepsilon} \subseteq \bar{B}_{\delta / \varepsilon} \backslash B_{(1-\sigma / 2) \delta / \varepsilon} .
$$

We construct a function that interpolates between $\overline{\mathbf{g}}_{\varepsilon, \delta}$ and $\overline{\mathbf{h}}$ on the annulus $A^{\varepsilon, \delta}:=B_{(1-\sigma / 2) \delta / \varepsilon} \backslash B_{(1-\sigma) \delta / \varepsilon}$. Let $\theta_{\delta}$ be a lifting for $\overline{\mathbf{g}}_{\delta}$, that is, a $\operatorname{map} \theta_{\delta} \in W^{1,2}\left(A_{\delta / 2, \delta} \backslash([0,+\infty) \times\{0\}) ; \mathbb{R}\right)$ such that $\overline{\mathbf{g}}_{\delta}=\exp \left(i \theta_{\delta}\right)$, and let $\theta_{\varepsilon, \delta}(x):=\theta_{\delta}(\varepsilon x)$. We also consider the function $\varphi(x):=\arctan \left(x_{2} / x_{1}\right)$, which is a lifting for $\overline{\mathbf{h}}$. Both $\theta_{\varepsilon, \delta}$ and $\varphi$ have a jump across the ray $[0,+\infty) \times$ $\{0\}$, and the size of both jumps is equal to ind $\left(\overline{\mathbf{g}}_{\varepsilon, \delta}, 0\right)=\operatorname{ind}(\overline{\mathbf{h}}, 0)=1$. Thus, $\theta_{\varepsilon, \delta}-\varphi \in W^{1,2}\left(A^{\varepsilon, \delta} ; \mathbb{R}\right)$. By combining a scaling argument, Lemma 17 and the continuity of the lifting in $W^{1 / 2,2}$ (see [13, Remark 3]) we deduce that, modulo rotations, there holds

$$
\left\|\theta_{\varepsilon, \delta}-\varphi\right\|_{W^{1 / 2,2}\left(\partial B_{(1-\sigma / 2) \delta / \varepsilon}\right)}=\left\|\theta_{\delta}-\varphi\right\|_{W^{1 / 2,2}\left(\partial B_{(1-\sigma / 2) \delta}\right)} \rightarrow 0
$$

as $\delta \searrow 0$. Let $u_{\varepsilon, \delta}$ be the unique solution of

$$
\left\{\begin{array}{l}
\Delta u=0 \quad \text { in } A^{\varepsilon, \delta} \\
u=\theta_{\varepsilon, \delta}-\varphi \quad \text { on } \partial B_{(1-\sigma / 2) \delta / \varepsilon}, \quad u=0 \quad \text { on } \partial B_{(1-\sigma) \delta / \varepsilon},
\end{array}\right.
$$

let $\psi_{\varepsilon, \delta}:=u_{\varepsilon, \delta}+\varphi$ and $\mathbf{u}_{\varepsilon, \delta}:=\mathbf{e}^{i \psi_{\varepsilon, \delta}}$. There holds $\mathbf{u}_{\varepsilon, \delta}=\overline{\mathbf{g}}_{\varepsilon}$ on $\partial B_{(1-\sigma / 2) \delta / \varepsilon}$, $\mathbf{u}_{\varepsilon, \delta}=\overline{\mathbf{h}}$ on $\partial B_{(1-\sigma) \delta / \varepsilon}$. By standard elliptic theory, we find that

$$
\left\|\nabla u_{\varepsilon, \delta}\right\|_{L^{2}\left(A^{\varepsilon, \delta}\right)}^{2} \leq C \sigma^{-1}\left\|\theta_{\varepsilon, \delta}-\varphi\right\|_{W^{1 / 2,2}\left(\partial B_{(1-\sigma / 2) \delta / \varepsilon}\right.}^{2} .
$$

The costant $C \sigma^{-1}$ can be obtained via a scaling argument; one could also solve (118) explicitely, passing to polar coordinates and using the method of separation of variables. Now, since $\left|\nabla \mathbf{u}_{\varepsilon, \delta}\right|=\left|\nabla \psi_{\varepsilon, \delta}\right|$ a.e. on $A^{\varepsilon, \delta}$, we have

$$
\left\|\nabla \mathbf{u}_{\varepsilon, \delta}\right\|_{L^{2}\left(A^{\varepsilon, \delta}\right)}^{2} \leq 2\left(\|\nabla \varphi\|_{L^{2}\left(A^{\varepsilon, \delta}\right)}^{2}+\left\|\nabla u_{\varepsilon, \delta}\right\|_{L^{2}\left(A^{\varepsilon, \delta}\right)}^{2}\right)
$$

Using (119), and computing explicitely the gradient of $\varphi$, we obtain

$$
\left\|\nabla \mathbf{u}_{\varepsilon, \delta}\right\|_{L^{2}\left(A^{\varepsilon, \delta}\right)}^{2} \leq C\left(\log \left(1+\frac{\sigma / 2}{1-\sigma}\right)+\sigma^{-1}\left\|\theta_{\varepsilon, \delta}-\varphi\right\|_{W^{1 / 2,2}\left(\partial B_{(1-\sigma / 2) \delta / \varepsilon}\right)}^{2}\right) .
$$

We choose

$$
\sigma=\sigma(\delta):=\left\|\theta_{\varepsilon, \delta}-\varphi\right\|_{W^{1 / 2,2}\left(\partial B_{(1-\sigma / 2) \delta / \varepsilon}\right)}+\delta
$$

Note that, thanks to (117), the right-hand side does not depend on $\varepsilon$ and converges to 0 as $\delta \searrow 0$; in particular, when $\delta$ is small enough, we have $\sigma \leq 1 / 2$. Then, using (120), we deduce that

$$
\lim _{\delta \searrow 0} \sup _{\varepsilon \in(0, \delta)}\left\|\nabla \mathbf{u}_{\varepsilon, \delta}\right\|_{L^{2}\left(A^{\varepsilon, \delta}\right)}^{2}=0 .
$$


Moreover, Lemma 16 combined with a scaling argument implies that

$$
\left\|\nabla \overline{\mathbf{g}}_{\varepsilon, \delta}\right\|_{L^{2}\left(B_{\delta / \varepsilon} \backslash B_{\delta / \varepsilon-\sigma(\delta) \delta /(2 \varepsilon)}\right)}^{2}=\left\|\nabla \overline{\mathbf{g}}_{\delta}\right\|_{L^{2}\left(B_{\delta} \backslash B_{\delta-\sigma(\delta) \delta / 2}\right)}^{2} \rightarrow 0
$$

as $\delta \searrow 0$.

Let $\mathbf{v}_{\delta}^{*} \in \mathrm{T}\left(\mathcal{S}_{\varepsilon} ; \mathbb{S}^{1}\right)$ be a minimizer for Problem $(114)$ on $B_{(\delta-\sigma(\delta) \delta) / \varepsilon}$, i.e. the problem that defines $\gamma_{2}(\varepsilon, \delta-\sigma(\delta) \delta)$. We construct the following discrete vector field:

$$
\mathbf{v}_{\varepsilon, \delta}:= \begin{cases}\mathbf{v}_{\delta}^{*} & \text { in } B_{(\delta-\sigma(\delta) \delta) / \varepsilon} \cap \mathcal{S}_{\varepsilon}^{0} \\ \mathbf{u}_{\varepsilon, \delta} & \text { in } A^{\varepsilon, \delta} \cap \mathcal{S}_{\varepsilon}^{0} \\ \mathbf{g}_{\varepsilon, \delta} & \text { in }\left(B_{\delta / \varepsilon} \backslash B_{\delta / \varepsilon-\sigma(\delta) \delta /(2 \varepsilon)}\right) \cap \mathcal{S}_{\varepsilon}^{0} .\end{cases}
$$

Thanks to standard interpolation arguments (see, e.g., [19, Theorem 3.1.5]), we see that

$$
\begin{aligned}
\frac{1}{2} \int_{\widehat{\left(B_{\delta / \varepsilon}\right)_{\varepsilon}}}\left|\nabla \mathbf{v}_{\varepsilon, \delta}\right|^{2} \mathrm{~d} S \leq \gamma_{2}(\varepsilon, \delta & -\sigma(\delta) \delta)+C\left\|\nabla \mathbf{u}_{\varepsilon, \delta}\right\|_{L^{2}\left(A^{\varepsilon, \delta}\right)}^{2} \\
& +C\left\|\nabla \overline{\mathbf{g}}_{\varepsilon, \delta}\right\|_{L^{2}\left(B_{\delta / \varepsilon} \backslash B_{\delta / \varepsilon-\sigma(\delta) \delta /(2 \varepsilon)}\right)}^{2}
\end{aligned}
$$

for some constant $C$ that does not depend on $\varepsilon, \delta$ (this is possible because the sequence of triangulations $\mathcal{S}_{\varepsilon}$ satisfies (112)). Then, with the help of (121), (122), we deduce that

$$
\frac{1}{2} \int_{\widehat{\left(B_{\delta / \varepsilon}\right)_{\varepsilon}}}\left|\nabla \mathbf{v}_{\varepsilon, \delta}\right|^{2} \mathrm{~d} S \leq \gamma_{2}(\varepsilon, \delta-\sigma(\delta) \delta)+r(\delta)
$$

where $r(\delta) \rightarrow 0$ and $\sigma(\delta) \rightarrow 0$ as $\delta \searrow 0$. However, due to (116), there holds $\mathbf{v}_{\varepsilon, \delta}=\overline{\mathbf{g}}_{\varepsilon, \delta}$ on $\partial_{\varepsilon} B_{\delta / \varepsilon}$, so $\mathbf{v}_{\varepsilon, \delta}$ is an admissible competitor in Problem (113) that defines $\gamma_{1}(\varepsilon, \delta)$. Thus, a comparison argument immediately yields the $\leq$-inequality in (115). The other inequality is obtained via a similar argument. Step 3. Remind that, in view of $\left(\mathrm{H}_{4}\right)$, the sequence $\mathcal{S}_{\varepsilon}$ converges to a triangulation $\mathcal{S}$. We consider the analogue of Problem (114) on $\mathcal{S}$, that is,

$$
\gamma_{3}(\varepsilon, \delta):=\min _{\mathbf{v} \in \mathrm{T}\left(\mathcal{S}, S^{1}\right)}\left\{\frac{1}{2} \int_{\widehat{\left(B_{\delta / \varepsilon}\right)_{*}}}|\nabla \widehat{\mathbf{v}}|^{2} \mathrm{~d} S: \quad \mathbf{v}=\overline{\mathbf{h}} \text { on } \partial_{*} B_{\delta / \varepsilon}\right\} .
$$

We have written $\widehat{\left(B_{\delta / \varepsilon}\right)}$ * to denote the union of the triangles $T \in \mathcal{S}$ such that $T \subseteq B_{\delta / \varepsilon}$, and $\partial_{*} B_{\delta / \varepsilon}:=\partial\left(\widehat{B_{\delta / \varepsilon}}\right) \cap \mathcal{S}^{0}$. We claim that there exists a positive number $s(\varepsilon, \delta)$ such that

$$
(1-s(\varepsilon, \delta)) \gamma_{3}(\varepsilon, \delta) \leq \gamma_{2}(\varepsilon, \delta) \leq(1+s(\varepsilon, \delta)) \gamma_{3}(\varepsilon, \delta)
$$

and

$$
\lim _{\varepsilon \searrow 0} s(\varepsilon, \delta)|\log \varepsilon|=0 \quad \text { for any } \delta .
$$


Thanks to $\left(\mathrm{H}_{4}\right)$ and Lemma 1 , for any $\varepsilon, \delta$ we find a quantity $s_{1}(\varepsilon, \delta)>0$ that satisfies $(125)$ and a piecewise affine map $\phi_{\varepsilon} \in \operatorname{Iso}\left(\mathcal{S}_{\varepsilon}, \mathcal{S}_{\mid B_{\delta / \varepsilon}}\right)$ such that

$$
\max \left\{\operatorname{Lip}\left(\phi_{\varepsilon}\right), \operatorname{Lip}\left(\phi_{\varepsilon}^{-1}\right)\right\} \leq 1+s_{1}(\varepsilon, \delta) .
$$

If $\mathbf{v}^{*}$ is a minimizer for Problem (123), then we have $\mathbf{v}^{*} \circ \phi_{\varepsilon}=\overline{\mathbf{h}} \circ \phi_{\varepsilon}$ on $\partial_{\varepsilon} B_{\delta / \varepsilon}$ so $\mathbf{v}^{*} \circ \phi_{\varepsilon}$ may not be admissible competitor for Problem (114). However, since $|\nabla \overline{\mathbf{h}}(x)| \leq C /|x|$, for any $i \in \partial_{\varepsilon} B_{\delta / \varepsilon}$ we have

$$
\left|\mathbf{v}^{*} \circ \phi_{\varepsilon}(i)-\overline{\mathbf{h}}(i)\right| \leq \frac{C \varepsilon}{\delta} d\left(\mathcal{S}_{\varepsilon}, \mathcal{S}_{\mid B_{\delta / \varepsilon}}\right)=: s_{2}(\varepsilon, \delta)
$$

and, thanks to $\left(\mathrm{H}_{4}\right), s_{2}(\varepsilon, \delta)$ also satisfies $(125)$. We set $s(\varepsilon, \delta):=s_{1}(\varepsilon, \delta)+$ $s_{2}(\varepsilon, \delta)$, and consider the discrete field

$$
\mathbf{v}_{\varepsilon, \delta}:= \begin{cases}\mathbf{v}^{*} & \text { in }\left(B_{\delta / \varepsilon} \cap \mathcal{S}_{\varepsilon}^{0}\right) \backslash \partial_{\varepsilon} B_{\delta / \varepsilon} \\ \overline{\mathbf{h}} & \text { on } \partial_{\varepsilon} B_{\delta / \varepsilon} .\end{cases}
$$

Then, $\mathbf{v}_{\varepsilon, \delta}$ is admissible for Problem (114), and a straightforward computation, based on (112), (126) and (127) yields that

$$
\begin{aligned}
\gamma_{2}(\varepsilon, \delta) & \leq \frac{1}{2} \int_{\widehat{\left(B_{\delta / \varepsilon}\right)_{\varepsilon}}}\left|\nabla \widehat{\mathbf{v}}_{\varepsilon, \delta}\right|^{2} \mathrm{~d} S \\
& \leq \frac{1+s(\varepsilon, \delta)}{2} \int_{\widehat{\left(B_{\delta / \varepsilon}\right)_{*}}}|\nabla \widehat{\mathbf{v}}|^{2} \mathrm{~d} S=(1+s(\varepsilon, \delta)) \gamma_{3}(\varepsilon, \delta) .
\end{aligned}
$$

Again, the other inequality in (124) follows by a similar argument.

Step 4 (Conclusion). Arguing as in [2, Theorem 4.1] (see also [12, Lemma III.1] for the continuous case), we find a number $\gamma(\bar{x})$ such that, for any $\delta$, we have

$$
\lim _{\varepsilon \searrow 0}\left(\gamma_{3}(\varepsilon, \delta)-\pi \log \frac{\delta}{\varepsilon}\right)=\gamma(\bar{x})
$$

Then, the proposition follows by combining (111), (115), (124) and (125).

Finally, we prepare the following refined lower bound on the Dirichlet energy of a unit norm vector field on an anulus.

Lemma 21 Let $C$ and $R^{*}$ be as in Lemma 9. Then, for any given $\rho_{1}<\rho_{2}<$ $R^{*}$ and any given tangent, unit norm vector field $\mathbf{w}$ defined in $A_{\rho_{1}, \rho_{2}}(\bar{x}):=$ $B_{\rho_{2}}(\bar{x}) \backslash B_{\rho_{1}}(\bar{x})$ with $\mathbf{w} \in W_{\tan }^{1,2}\left(A_{\rho_{1}, \rho_{2}}(\bar{x}) ; \mathbb{S}^{2}\right)$ and with ind $(\mathbf{w}, \bar{x})=d$, we have

$$
\frac{1}{2} \int_{\partial B_{\rho}(\bar{x})}|\mathrm{Dw}|^{2} \mathrm{~d} s \geq \frac{1}{4 \pi \rho+C \rho^{2}}\left|2 \pi d-\int_{B_{\rho}(\bar{x})} G \mathrm{~d} s\right|^{2}
$$

for any $\rho \in\left(\rho_{1}, \rho_{2}\right)$. 
Proof. The proof is based on the fact that for a vector field w with the regularity of the statement there holds (see (43))

$$
|\mathrm{D} \mathbf{w}|^{2}=|\jmath(\mathbf{w})|^{2} .
$$

Then, the proof is similar, actually simpler, to the proof of Lemma 9. In particular, there exist positive numbers $R_{*}$ and $C$ such that, for any $x_{0} \in M$ and any $0<\rho \leq R_{*}$, there holds

$$
\mathscr{H}^{1}\left(\partial B_{\rho}\left(x_{0}\right)\right) \leq 2 \pi \rho+C \rho^{2} .
$$

Thus, Jensen's inequality gives

$$
\frac{1}{2} \int_{\partial B_{\rho}(\bar{x})}|\mathrm{D} \mathbf{w}|^{2} \mathrm{~d} s=\frac{1}{2} \int_{\partial B_{\rho}(\bar{x})}|\jmath(\mathbf{w})|^{2} \mathrm{~d} s \geq \frac{1}{4 \pi \rho+C \rho^{2}}\left|2 \pi d-\int_{B_{\rho}} G\right|^{2} .
$$

Lemma 22 For any $\rho_{1}, \rho_{2} \in\left(0, R^{*}\right)$, for any $\bar{x} \in M$ and for any $\mathbf{w} \in$ $W_{\tan }^{1,2}\left(A_{\rho_{1}, \rho_{2}}(\bar{x}) ; \mathbb{S}^{2}\right)$, there holds

$$
\frac{1}{2} \int_{A_{\rho_{1}, \rho_{2}}(\bar{x})}|\mathrm{D} \mathbf{w}|^{2} \mathrm{~d} S \geq \pi|d|^{2} \log \frac{\rho_{2}}{\rho_{1}}-C \pi|d|^{2} \log \frac{2 \pi+C \rho_{2}}{2 \pi+C \rho_{1}}-|d|,
$$

where $d:=\operatorname{ind}(\mathbf{w}, \bar{x})$.

Proof. Lemma 21 gives that

$$
\frac{1}{2} \int_{\partial B_{\rho}(\bar{x})}|\mathrm{Dw}|^{2} \mathrm{~d} s \geq \frac{2 \pi^{2} d^{2}}{2 \pi \rho+C \rho^{2}}-\frac{|d|}{\rho} \int_{B_{\rho}(\bar{x})} G d S .
$$

To conclude, we integrate between $\rho_{1}$ and $\rho_{2}$ and note that thanks to the smoothness of the Gauss curvature $G$, by possibly reducing $R^{*}$, we can assume that

$$
\int_{\rho_{1}}^{\rho_{2}}\left(\frac{1}{\rho} \int_{B_{\rho}(\bar{x})} G \mathrm{~d} S\right) \mathrm{d} \rho<1 .
$$

\subsection{Proof of Theorem B}

Proof of (i) - Compactness. The proof follows the line of [2, Theorem 4.2]. Given $\mathbf{v}_{\varepsilon} \in \mathrm{T}\left(\mathcal{T}_{\varepsilon} ; \mathbb{S}^{2}\right)$ such that

$$
X Y_{\varepsilon}\left(\mathbf{v}_{\varepsilon}\right)-\mathscr{K} \pi|\log \varepsilon| \leq C
$$

the existence of a subsequence of $\hat{\mu}_{\varepsilon}$ and of the measure $\mu \in X$ with $\sum_{i=1}^{k}\left|d_{i}\right| \leq$ $\mathscr{K}$ follows from the compactness part of the zeroth order $\Gamma$-convergence result in Theorem A. Thus, we are left with the proof of the implication

$$
\sum_{i=1}^{k}\left|d_{i}\right|=\mathscr{K} \quad \Longrightarrow \quad\left|d_{i}\right|=1 \text { for any } i=1, \ldots, \mathscr{K},
$$


which implies that

$$
\mathscr{K} \equiv \chi(M) \bmod 2 .
$$

Now, fix a small $r>0$ in such a way that the balls $B_{r}\left(x_{i}\right), i=1, \ldots, \mathscr{K}$ are pairwise disjoint. As usual we set

$$
M_{r}:=M \backslash \bigcup_{i=1}^{\mathscr{K}} B_{r}\left(x_{i}\right) .
$$

We have

$$
X Y_{\varepsilon}\left(\mathbf{v}_{\varepsilon}\right)=\frac{1}{2} \sum_{i=1}^{k}\left|\mathbf{w}_{\varepsilon}\right|_{W_{\varepsilon}^{1,2}\left(B_{r}\left(x_{i}\right)\right)}^{2}+\frac{1}{2}\left|\mathbf{w}_{\varepsilon}\right|_{W_{\varepsilon}^{1,2}\left(M_{r}\right)}^{2} .
$$

Thanks to the localized $\Gamma$-liminf inequality in Proposition 4, the first term in the equality above is bounded from below in the following way:

$$
\frac{1}{2} \sum_{i=1}^{k}\left|\mathbf{w}_{\varepsilon}\right|_{W_{\varepsilon}^{1,2}\left(B_{r}\left(x_{i}\right)\right)}^{2} \geq \pi \sum_{i=1}^{k}\left|d_{i}\right| \log \frac{r}{\varepsilon}+C
$$

for a constant $C$ that does not depend on $r$. Thus, the energy estimate (23) and the fact that $\sum_{i}\left|d_{i}\right|=\mathscr{K}$ give

$$
\frac{1}{2}\left|\mathbf{w}_{\varepsilon}\right|_{W_{\varepsilon}^{1,2}\left(M_{r}\right)}^{2} \leq \mathscr{K} \pi|\log r|+C .
$$

Consequently, we have that the sequence $\mathbf{w}_{\varepsilon}$ is uniformly bounded (w.r.t. $\varepsilon$ ) in $W_{\varepsilon}^{1,2}\left(M_{r}\right)$ for any $r>0$. Hence, there exists a tangent vector field $\mathbf{v}$ and a subsequence such that

$$
\mathbf{w}_{\varepsilon} \rightarrow \mathbf{v} \text { strongly in } L^{2}\left(M ; \mathbb{R}^{3}\right) \text { and weakly in } W_{\text {loc }}^{1,2}\left(M \backslash \bigcup_{i=1}^{\mathscr{K}} x_{i} ; \mathbb{R}^{3}\right) \text {. }
$$

Moreover, thanks to Lemma 5 and to the strong $L^{2}$ convergence we have that $|\mathbf{v}|=1$. Passing to the limit as $\varepsilon \searrow 0$ in (132), we also see that $\mathbb{W}(\mathbf{v})<+\infty$. Finally, thanks to Fubini's Theorem, we can find some $r^{\prime} \in(r, 2 r)$ such that for any $i=1, \ldots, k$

$$
\int_{\partial B_{r^{\prime}}\left(x_{i}\right)}\left|\nabla_{\mathrm{s}} \mathbf{w}_{\varepsilon}\right|^{2} \mathrm{~d} s \leq C .
$$

implying, by compactness, that

$$
\mathbf{w}_{\varepsilon} \rightarrow \mathbf{v} \text { uniformly on } \partial B_{r^{\prime}}\left(x_{i}\right)
$$

and then we have that

$$
\operatorname{ind}\left(\mathbf{v}, x_{i}\right)=d_{i} .
$$

Thus, recalling that $\mathbf{v} \in W_{\tan }^{1,1}\left(M ; \mathbb{S}^{2}\right)$ (see Lemma 15) we conclude thanks to Lemma 6 that $\star \mathrm{d} \jmath(\mathbf{v})=2 \pi \mu-G \mathrm{~d} S$. Now, we prove that $k=\mathscr{K} \equiv \chi(M)$ mod 2 and that $\left|d_{i}\right|=1$ for any $i=1, \ldots, \mathscr{K}$. We fix $\rho_{1}$ and $\rho_{2}$ in the interval 
$\left(0, R^{*}\right]\left(R^{*}\right.$ is as in Lemma 9) with $\rho_{1}<\rho_{2}$ and such that the geodesic balls $B_{\rho_{2}}\left(x_{i}\right)$ are pairwise disjoint. We have

$$
\begin{aligned}
X Y_{\varepsilon}\left(\mathbf{v}_{\varepsilon}\right) & =\frac{1}{2} \sum_{i=1}^{k}\left|\mathbf{w}_{\varepsilon}\right|_{W_{\varepsilon}^{1,2}\left(B_{\rho_{1}}\left(x_{i}\right)\right)}^{2}+\frac{1}{2}\left|\mathbf{w}_{\varepsilon}\right|_{W_{\varepsilon}^{1,2}\left(M \backslash \bigcup_{i=1}^{k} B_{\rho_{1}}\left(x_{i}\right)\right)}^{2} \\
& \geq \frac{1}{2} \sum_{i=1}^{k}\left|\mathbf{w}_{\varepsilon}\right|_{W_{\varepsilon}^{1,2}\left(B_{\rho_{1}}\left(x_{i}\right)\right)}^{2}+\frac{1}{2} \sum_{i=1}^{k}\left|\mathbf{w}_{\varepsilon}\right|_{W_{\varepsilon}^{1,2}\left(A_{\rho_{1}, \rho_{2}}^{i}\right)}^{2} .
\end{aligned}
$$

Now, thanks to the localized $\Gamma$-lim inf inequality in Proposition 4 , the first term in the inequality above is, as in (131), bounded from below

$$
\frac{1}{2} \sum_{i=1}^{k}\left|\mathbf{w}_{\varepsilon}\right|_{W_{\varepsilon}^{1,2}\left(B_{\rho_{1}}\left(x_{i}\right)\right)}^{2} \geq \pi \sum_{i=1}^{k}\left|d_{i}\right| \log \frac{\rho_{1}}{\varepsilon}+C .
$$

Thus, we get (recall that $\sum_{i=1}^{k}\left|d_{i}\right|=\mathscr{K}$ )

$$
\frac{1}{2} \sum_{i=1}^{k}\left|\mathbf{w}_{\varepsilon}\right|_{W_{\varepsilon}^{1,2}\left(A_{\rho_{1}, \rho_{2}}^{i}\right)}^{2} \leq X Y_{\varepsilon}\left(\mathbf{v}_{\varepsilon}\right)-\pi \mathscr{K}|\log \varepsilon| \leq C_{\mathscr{K}} .
$$

As before, we obtain that the sequence $\mathbf{w}_{\varepsilon}$ is uniformly bounded (w.r.t. $\varepsilon$ ) $W_{\varepsilon}^{1,2}\left(\bigcup_{i=1}^{k} A_{\rho_{1}, \rho_{2}}^{i}\right)$. Let $\mathbf{v}$ the unit norm vector field identified above. By semicontinuity of the norm we get

$$
\begin{aligned}
\liminf _{\varepsilon \rightarrow 0} \frac{1}{2} \sum_{i=1}^{k}\left|\mathbf{w}_{\varepsilon}\right|_{W_{\varepsilon}^{1,2}\left(A_{\rho_{1}, \rho_{2}}^{i}\right)}^{2} \geq \frac{1}{2} \sum_{i=1}^{k} \int_{A_{\rho_{1}, \rho_{2}}^{i}}|\mathrm{D} \mathbf{v}|^{2} \mathrm{~d} S & \\
& +\frac{1}{2} \sum_{i=1}^{k} \int_{A_{\rho_{1}, \rho_{2}}^{i}}|\mathrm{~d} \boldsymbol{\gamma}[\mathbf{v}]|^{2} \mathrm{~d} S .
\end{aligned}
$$

Thus,

$$
\begin{array}{r}
X Y_{\varepsilon}\left(\mathbf{v}_{\varepsilon}\right) \geq \pi \sum_{i=1}^{k} \mid \\
\left|d_{i}\right| \log \frac{\rho_{1}}{\varepsilon}+\frac{1}{2} \sum_{i=1}^{k} \int_{A_{\rho_{1}, \rho_{2}}^{i}}|\mathrm{D} \mathbf{v}|^{2} \mathrm{~d} S \\
+\frac{1}{2} \sum_{i=1}^{k} \int_{A_{\rho_{1}, \rho_{2}}^{i}}|\mathrm{~d} \boldsymbol{\gamma}[\mathbf{v}]|^{2} \mathrm{~d} S+C
\end{array}
$$

Moreover, Lemma 22, together with $\sum_{i=1}^{k}\left|d_{i}\right|=\mathscr{K}$, gives

$$
\begin{array}{r}
X Y_{\varepsilon}\left(\mathbf{v}_{\varepsilon}\right) \geq \pi \mathscr{K}|\log \varepsilon|+\pi \sum_{i=1}^{k}\left(\left|d_{i}\right|^{2}-\left|d_{i}\right|\right) \log \frac{\rho_{2}}{\rho_{1}} \\
-C \pi \sum_{i=1}^{k}\left|d_{i}\right|^{2} \log \frac{2 \pi+C \rho_{2}}{2 \pi+C \rho_{1}}+C .
\end{array}
$$


Thus, letting $\rho_{1} \rightarrow 0$ and using the energy bound, we get that

$$
\lim _{\rho_{1} \rightarrow 0} \pi \sum_{i=1}^{k}\left(\left|d_{i}\right|^{2}-\left|d_{i}\right|\right) \log \frac{\rho_{2}}{\rho_{1}} \leq C_{\mathscr{K}}+C+C \pi \sum_{i=1}^{k}\left|d_{i}\right|^{2} \log \frac{2 \pi+C \rho_{2}}{2 \pi}
$$

Then, since the last term is bounded by a constant depending on $\mathscr{K}$ and on $R^{*}$ we get that $\left|d_{i}\right|=1$ for any $i=1, \ldots, \mathscr{K}$ and thus $\mathbf{v} \in \mathcal{V}_{\mathscr{K}}$.

Proof of (ii) $-\Gamma$-liminf. Let $\mathbf{v}_{\varepsilon}$ be a sequence in $\mathrm{T}\left(\mathcal{T}_{\varepsilon} ; \mathbb{S}^{2}\right)$ satisfying the energy estimate (see (23))

$$
X Y_{\varepsilon}\left(\mathbf{v}_{\varepsilon}\right)-\mathscr{K} \pi|\log \varepsilon| \leq C
$$

with $\mathscr{K} \equiv \chi(M) \bmod 2$. Let $\mathbf{v} \in \mathcal{V}_{\mathscr{K}}$ be a tangent unit-norm vector field such that

$$
\hat{\mu}_{\varepsilon}\left(\mathbf{v}_{\varepsilon}\right) \stackrel{\text { flat }}{\longrightarrow} \star \mathrm{d} \jmath(\mathbf{v})
$$

and

$$
\mathbf{w}_{\varepsilon} \rightarrow \mathbf{v} \quad \text { strongly in } L^{2}\left(M ; \mathbb{R}^{3}\right) \text { and weakly in } W_{\text {loc }}^{1,2}\left(M \backslash \bigcup_{i=1}^{\mathscr{K}} x_{i} ; \mathbb{R}^{3}\right) .
$$

In particular, the semicontinuity of the norm gives that, for any $r>0$ such that the geodesic balls $B_{2 r}\left(x_{i}\right)$ are pairwise disjoint, there holds (recall (130))

$$
\liminf _{\varepsilon \rightarrow 0} \frac{1}{2}\left|\mathbf{w}_{\varepsilon}\right|_{W_{\varepsilon}^{1,2}\left(M_{r}\right)}^{2} \geq \frac{1}{2} \int_{M_{r}}|\mathrm{D} \mathbf{v}|^{2}+|\mathrm{d} \gamma[\mathbf{v}]|^{2} \mathrm{~d} S .
$$

In what follows, we will assume that $d_{i}=1$ for any $i$. The case $d_{i}=-1$ for some index $i$ can be treated in a similar way, with straightforward modifications of the proof.

For any $i$ we consider the minimum problem (103) with $\bar{x}=x_{i}$ and $t \leq r$. As in [2], the following property holds. For any fixed $\sigma>0$, there exists a positive $\omega=\omega(\sigma)$ (independent of $t$ and of $i$ ) such that if

$$
\sigma<d\left(\mathbf{w}_{\varepsilon}, \mathcal{H}\left(t, x_{i}\right)\right):=\inf \left\{\left\|\nabla_{\mathbf{s}} \mathbf{w}_{\varepsilon}-\nabla_{\mathbf{s}} \mathbf{v}\right\|_{L^{2}\left(A_{t / 2, t}\left(x_{i}\right)\right)}: \mathbf{v} \in \mathcal{H}\left(t, x_{i}\right)\right\} .
$$

then

$$
\liminf _{\varepsilon \rightarrow 0} \frac{1}{2}\left|\mathbf{w}_{\varepsilon}\right|_{W_{\varepsilon}^{1,2}\left(A_{t / 2, t}\left(x_{i}\right)\right)}^{2} \geq \omega(\sigma)+\eta\left(t, x_{i}\right) \quad \text { for any } i=1, \ldots, \mathscr{K},
$$

where $\eta\left(t, x_{i}\right)$ is the minimum value for (103), namely

$$
\begin{gathered}
\eta\left(t, x_{i}\right):=\min _{\mathbf{w} \in W_{\tan }^{1,2}\left(A_{t / 2, t}\left(x_{i}\right) ; \mathbb{S}^{2}\right)}\left\{\frac{1}{2} \int_{A_{t / 2, t}\left(x_{i}\right)}|\mathrm{D} \mathbf{w}|^{2}+|\mathrm{d} \gamma[\mathbf{w}]|^{2} \mathrm{~d} S,\right. \\
\left.\operatorname{ind}\left(\mathbf{w}, x_{i}\right)=1\right\} .
\end{gathered}
$$


By Lemma 18, if $t \leq r$ is sufficiently small we have

$$
\eta\left(t, x_{i}\right) \geq \frac{\pi}{2} \log 2
$$

Then, we fix $L \in \mathbb{N}$ in such a way that

$$
L \omega(\sigma) \geq \mathbb{W}(\mathbf{v})+\sum_{i=1}^{\mathscr{K}} \gamma\left(x_{i}\right)-\mathscr{K}(\pi \log r+C) .
$$

where $C$ is the constant that appears in the localized liminf inequality in Proposition 4 . This is clearly possible since $\mathbb{W}(\mathbf{v})<+\infty$. Moreover, we set $\lambda:=2^{1 /(2 \mathscr{K})} \in[1,2]$. For $l=1, \ldots, L$, and $i=1, \ldots, \mathscr{K}$ we set $A_{l}^{i}:=$ $B_{\lambda^{1-l_{r}}}\left(x_{i}\right) \backslash B_{\lambda^{-l} r}\left(x_{i}\right)$.

We have to face the two following situations.

Case 1 For a $\varepsilon$ sufficiently small and for any $l=1, \ldots, L$, there exists one $i$ such that $d\left(\mathbf{w}_{\varepsilon}, \mathcal{H}\left(\lambda^{1-l} r, x_{i}\right)\right) \geq \sigma$. Thus, thanks to (141), (143), to the localized lim inf inequality in Proposition 4 and to (144), we get (recall that $\mathscr{K} \geq 1)$

$$
\begin{aligned}
X Y_{\varepsilon}\left(\mathbf{v}_{\varepsilon}\right) & \geq \sum_{i=1}^{\mathscr{K}} \frac{1}{2}\left|\mathbf{w}_{\varepsilon}\right|_{W_{\varepsilon}^{1,2}\left(B_{\lambda}-L_{r}\left(x_{i}\right)\right)}^{2}+\sum_{l=1}^{L} \sum_{i=1}^{\mathscr{K}} \frac{1}{2}\left|\mathbf{w}_{\varepsilon}\right|_{W_{\varepsilon}^{1,2}\left(A_{l}^{i}\right)}^{2} \\
& \geq \pi \mathscr{K} \log \frac{\lambda^{-L} r}{\varepsilon}-C \mathscr{K}+\frac{\pi}{2} L \log 2+L \omega(\sigma)+\mathrm{o}_{\varepsilon \rightarrow 0}(1) \\
& \geq \pi \mathscr{K}|\log \varepsilon|+\sum_{i=1}^{\mathscr{K}} \gamma\left(x_{i}\right)+\mathbb{W}(\mathbf{v})+\mathrm{o}_{\varepsilon \rightarrow 0}(1) .
\end{aligned}
$$

Case 2 The second possibility we have to face is that (up to a subsequence) there exists a $\bar{l} \in\{1, \ldots, L\}$ such that for every $i$ there holds

$$
d\left(\mathbf{w}_{\varepsilon}, \mathcal{H}\left(\lambda^{1-\bar{l}} r, x_{i}\right)\right) \leq \sigma
$$

Now, for any $i=1, \ldots, \mathscr{K}$, let $\mathbf{w}_{\varepsilon}^{i}$ a vector field in $\mathcal{H}$ such that

$$
d\left(\mathbf{w}_{\varepsilon}, \mathcal{H}\left(\lambda^{1-\bar{l}} r, x_{i}\right)\right)=\left\|\nabla_{\mathbf{S}} \mathbf{w}_{\varepsilon}-\nabla_{\mathbf{S}} \mathbf{w}_{\varepsilon}^{i}\right\|_{L^{2}\left(A_{l}^{i}\right)} .
$$

Note that by construction, $\mathbf{w}_{\varepsilon}^{i}$ is a tangent vector field with unit norm (defined in $\left.A_{l}^{i}\right)$ and such that ind $\left(\mathbf{w}_{\varepsilon}^{i}, x_{i}\right)=1$. Thus, by mimicking the cut off argument in [2], we can construct a discrete vector field $\tilde{\mathbf{v}}_{\varepsilon} \in \mathrm{T}\left(\mathcal{T}_{\varepsilon} ; \mathbb{S}^{2}\right)$ for which its corresponding $\tilde{\mathbf{w}}_{\varepsilon}$ (see (36)) verifies for any $i=1, \ldots, \mathscr{K} \tilde{\mathbf{w}}_{\varepsilon}=\mathbf{w}_{\varepsilon}^{i}$ on $\partial B_{\lambda^{1-\bar{\tau}_{r}}}\left(x_{i}\right)$ and

$$
\frac{1}{2}\left|\mathbf{w}_{\varepsilon}\right|_{W_{\varepsilon}^{1,2}\left(B_{\lambda^{1}-\bar{l}_{r}}\left(x_{i}\right)\right)}^{2} \geq \frac{1}{2}\left|\tilde{\mathbf{w}}_{\varepsilon}\right|_{W_{\varepsilon}^{1,2}\left(B_{\lambda^{1}-\bar{l}_{r}}\left(x_{i}\right)\right)}^{2}+\mathfrak{r}(\varepsilon, \sigma),
$$

with $\lim _{\sigma \searrow 0} \lim _{\varepsilon \searrow 0} \mathfrak{r}(\varepsilon, \sigma)=0$. To construct such a vector field, one can map the lattice $\mathcal{T}_{0}^{\varepsilon}$ on $\mathbb{R}^{2}$ with geodesic normal coordinates and then use the 
construction of [2] (see also [3] for an analogous construction in the framework of the two dimensional Ginzburg Landau functional).

We are ready to conclude the proof of the $\Gamma$-lim inf. On the one hand, the construction above and Proposition 5 give that (we set $\bar{r}:=\lambda^{1-\bar{l}} r$ and we recall that $\gamma_{x_{i}}(\varepsilon, \bar{r})$ is the value of the minimum problem in (104))

$$
\begin{aligned}
\frac{1}{2} \sum_{i=1}^{\mathscr{K}}\left|\mathbf{w}_{\varepsilon}\right|_{W_{\varepsilon}^{1,2}\left(B_{\bar{r}}\left(x_{i}\right)\right)}^{2} & \geq \frac{1}{2} \sum_{i=1}^{\mathscr{K}}\left|\overline{\mathbf{w}}_{\varepsilon}\right|_{W_{\varepsilon}^{1,2}\left(B_{\lambda^{1}-\bar{l}_{r}}\left(x_{i}\right)\right)}^{2}+\mathfrak{r}(\varepsilon, \sigma) \\
& \geq \sum_{i=1}^{\mathscr{K}} \gamma_{x_{i}}(\varepsilon, \bar{r})+\mathfrak{r}(\varepsilon, \sigma) \\
& =\sum_{i=1}^{\mathscr{K}} \gamma\left(x_{i}\right)+\pi \mathscr{K}\left|\log \frac{\varepsilon}{\bar{r}}\right|+\mathfrak{r}(\varepsilon, \sigma)+\mathrm{o}_{\varepsilon \rightarrow 0}(1) \\
& +\mathrm{o}_{\bar{r} \rightarrow 0}(1) .
\end{aligned}
$$

On the other hand, (140) and (18) give

$$
\begin{aligned}
\frac{1}{2}\left|\mathbf{w}_{\varepsilon}\right|_{W_{\varepsilon}^{1,2}\left(M_{\bar{r}}\right)}^{2} & \geq \frac{1}{2} \int_{M_{\bar{r}}}|\mathrm{D} \mathbf{v}|^{2} \mathrm{~d} S+\frac{1}{2} \int_{M_{\bar{r}}}|\mathrm{~d} \gamma[\mathbf{v}]|^{2} \mathrm{~d} S+\mathrm{o}_{\varepsilon \rightarrow 0}(1) \\
& \geq \pi \mathscr{K}|\log \bar{r}|+\mathbb{W}(\mathbf{v})+\mathrm{o}_{\varepsilon \rightarrow 0}(1)+\mathrm{o}_{\bar{r} \rightarrow 0}(1) .
\end{aligned}
$$

As a result, combining (147) and (148) we get

$$
\begin{aligned}
X Y_{\varepsilon}\left(\mathbf{v}_{\varepsilon}\right)= & \frac{1}{2} \sum_{i=1}^{\mathscr{K}}\left|\mathbf{w}_{\varepsilon}\right|_{W_{\varepsilon}^{1,2}\left(B_{\bar{r}}\left(x_{i}\right)\right)}^{2}+\frac{1}{2}\left|\mathbf{w}_{\varepsilon}\right|_{W_{\varepsilon}^{1,2}\left(M_{\bar{r}}\right)}^{2} \\
\geq & \pi \mathscr{K}|\log \bar{r}|+\mathbb{W}(\mathbf{v})+\sum_{i=1}^{\mathscr{K}} \gamma\left(x_{i}\right)+\pi \mathscr{K}\left|\log \frac{\varepsilon}{\bar{r}}\right| \\
& +\mathfrak{r}(\varepsilon, \sigma)+\mathrm{o}_{\varepsilon \rightarrow 0}(1)+\mathrm{o}_{\bar{r} \rightarrow 0}(1) \\
= & \pi \mathscr{K}|\log \varepsilon|+\mathbb{W}(\mathbf{v})+\sum_{i=1}^{\mathscr{K}} \gamma\left(x_{i}\right)+\mathfrak{r}(\varepsilon, \sigma)+\mathrm{o}_{\varepsilon \rightarrow 0}(1)+\mathrm{o}_{\bar{r} \rightarrow 0}(1) .
\end{aligned}
$$

Thus, sending $\varepsilon \rightarrow 0, \sigma \rightarrow 0, r \rightarrow 0$ we get the $\Gamma$-liminf inequality (26).

Proof of (iii) $-\Gamma$-limsup. Given $\mathbf{v} \in \mathcal{V}_{\mathscr{K}}$, the goal is to construct a sequence $\mathbf{v}_{\varepsilon}$ such that $\mathbf{w}_{\varepsilon} \rightarrow \mathbf{v}$ weakly in $L^{2}\left(M ; \mathbb{R}^{3}\right)$, such that $\hat{\mu}_{\varepsilon}\left(\mathbf{v}_{\varepsilon}\right) \stackrel{\text { flat }}{\longrightarrow} \star \mathrm{d} \jmath(\mathbf{v})$ and such that the limsup inequality $(27)$ holds. The recovery sequence $\mathbf{v}_{\varepsilon}$ is constructed as in [2]. For the sake of clarity, we highlight the main points. First of all we suppose that $\mathbf{v}$ is smooth, otherwise (as in [2]) we can approximate it with a smooth vector field in the $W^{1,2}$ norm (see [44] and [18]). Then, we recall (102) that gives that, for a fixed $\rho>0$,

$$
\lim _{j \rightarrow+\infty} \frac{1}{2} \int_{A_{2^{-j-1} \rho_{\rho, 2}-j \rho}^{i}}|\mathrm{D} \mathbf{v}|^{2}+|\mathrm{d} \gamma[\mathbf{v}]|^{2} \mathrm{~d} S=\pi \log 2 .
$$


Thus, Lemma 19 gives that we can find a matrix $R=R(j) \in \mathrm{SO}(2)$ such that for any $i$

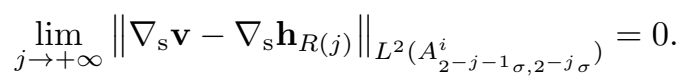

Now, we construct a tangent vector field on $A_{2^{-j-1} \sigma, 2^{-j} \sigma}^{i}$ that interpolates between $\mathbf{h}_{R(j)}$ and $\mathbf{v}$ on $\partial B_{2^{-j-1} \rho}\left(x_{i}\right)$ and $\partial B_{2^{-j} \rho}\left(x_{i}\right)$, respectively. Let $\psi$ : $\left[\frac{1}{2}, 1\right] \rightarrow \mathbb{R}$ be a smooth cut off function such that $\psi(1 / 2)=0$ and $\psi(1)=1$. Let $\theta_{\overline{\mathbf{v}}}$ and $\theta_{\overline{\mathbf{h}}_{R(j)}}$ be the liftings of the vector fields $\varphi^{*} \mathbf{v}$ and $\varphi^{*} \mathbf{h}_{R(j)}$. We set $u_{j}^{i}:=\psi\left(2^{j} \rho|x|\right) \theta_{\overline{\mathbf{v}}}+\left(1-\psi\left(2^{j} \rho|x|\right)\right) \theta_{\overline{\mathbf{h}}_{R(j)}}$, and then we set $\overline{\mathbf{u}}_{j}^{i}:=\mathbf{e}^{\iota u_{j}^{i}}, \iota$ being the immaginary unit. Finally, we map $\overline{\mathbf{u}}_{j}^{i}$ back on $M$ using $\varphi_{*}$, namely we set $\mathbf{u}_{j}^{i}:=\varphi_{*} \overline{\mathbf{u}}_{j}^{i}$. Using (149) it is not difficult to see that for any $i=1, \ldots$, there holds that

$$
\lim _{j \rightarrow+\infty} \int_{A_{2^{-j-1} \sigma, 2^{-j} \sigma}^{i}}\left|\nabla_{\mathbf{s}} \mathbf{u}_{j}^{i}\right|^{2} \mathrm{~d} S=\pi \log 2 .
$$

We consider the following sequence of vector fields

$$
\mathbf{v}_{j}:= \begin{cases}\mathbf{v} & \text { in } M \backslash \bigcup_{i=1}^{\mathscr{K}} B_{2^{-j-1}} \rho\left(x_{i}\right) \\ \tilde{\mathbf{u}}_{j}^{i} & \text { in } A_{2^{-j-1} \rho, 2^{-j}}^{i} .\end{cases}
$$

Now, for any $i \in\{1, \ldots, \mathscr{K}\}$ we consider the discrete vector field $\mathbf{v}_{\varepsilon}^{i}:=\varphi_{*} \overline{\mathbf{v}}_{\varepsilon}^{i}$ with $\overline{\mathbf{v}}_{\varepsilon}^{i}$ be the minimizer of (114) in $B_{2^{-j-1} \rho}$ with $\overline{\mathbf{h}_{R(j)}}$ as boundary condition on $\partial_{\varepsilon} B_{2^{-j-1} \rho}$. Finally, we let the recovery sequence be the sequence of discrete vector fields that coincides with $\mathbf{v}_{j}$ on the nodes of $M \backslash \bigcup_{i=1}^{\mathscr{K}} B_{2^{-j-1} \rho}\left(x_{i}\right)$ and with $\mathbf{v}_{\varepsilon}^{i}$ on the nodes of $B_{2^{-j-1}} \rho$. More precisely, we set

$$
\mathbf{v}_{\varepsilon, j}:=\left\{\begin{aligned}
\mathbf{v}_{j} & \text { in }\left(M \backslash \bigcup_{i=1}^{\mathscr{K}} B_{2^{-j-1}} \rho\left(x_{i}\right)\right) \cap \mathcal{T}_{\varepsilon}^{0}, \\
\mathbf{v}_{\varepsilon}^{i} & \text { in } B_{2^{-j-1} \rho}\left(x_{i}\right) \cap \mathcal{T}_{\varepsilon}^{0} \text { for } i=1, \ldots, \mathscr{K} .
\end{aligned}\right.
$$

Now, let $\mathbf{w}_{\varepsilon, j}:=\hat{\mathbf{v}}_{\varepsilon, j} \circ \widehat{P}_{\varepsilon}^{-1}$ as in (36). By a diagonal argument, we have that there exists sequence $j(\varepsilon) \stackrel{\varepsilon \rightarrow 0}{\longrightarrow} 0$ such that

$$
\mathbf{w}_{\varepsilon, j(\varepsilon)} \rightarrow \mathbf{v} \text { strongly in } L^{2}\left(M ; \mathbb{R}^{3}\right) \text { and weakly in } W_{\text {loc }}^{1,2}\left(M \backslash \bigcup_{i=1}^{\mathscr{K}} x_{i} ; \mathbb{R}^{3}\right) .
$$


Moreover, $\hat{\mu}_{\varepsilon}\left(\mathbf{v}_{\varepsilon, j(\varepsilon)}\right) \stackrel{\text { flat }}{\longrightarrow} \star \mathrm{d} \jmath(\mathbf{v})$. Finally, along the same sequence $j(\varepsilon) \mathbf{v}_{\varepsilon, j(\varepsilon)}$ is indeed a recovery sequence. In fact the following holds

$$
\begin{aligned}
& X Y_{\varepsilon}\left(\mathbf{v}_{\varepsilon, j}\right)-\pi \mathscr{K}|\log \varepsilon|=\frac{1}{2}\left|\mathbf{w}_{\varepsilon, j}\right|_{W_{\varepsilon}^{1,2}(M)}^{2}-\pi \mathscr{K}|\log \varepsilon| \\
& =\frac{1}{2}|\mathbf{v}|_{W_{\varepsilon}^{1,2}\left(M \backslash \bigcup_{i=1}^{\mathscr{K}} B_{2}-j_{\rho}\left(x_{i}\right)\right)}^{2}-\pi \mathscr{K}\left|\log 2^{-j} \rho\right|
\end{aligned}
$$

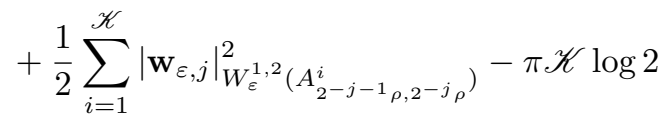

$$
\begin{aligned}
& +\frac{1}{2} \sum_{i=1}^{\mathscr{K}}\left|\mathbf{w}_{\varepsilon, j}\right|_{W_{\varepsilon}^{1,2}\left(B_{2^{-j-1} \rho}\left(x_{i}\right)\right)}^{2}-\pi \mathscr{K}\left|\log \frac{\varepsilon}{2^{-j-1} \rho}\right| .
\end{aligned}
$$

Thus, using (150), (151), and Proposition 5 we conclude.

Acknowledgements G.C.'s research has received funding from the European Research Council under the European Union's Seventh Framework Programme (FP7/2007-2013) / ERC grant agreement $\mathrm{n}^{\circ}$ 291053. G.C. was also partially supported by the Basque Government through the BERC 2014-2017 program; and by the Spanish Ministry of Economy and Competitiveness MINECO: BCAM Severo Ochoa accreditation SEV-2013-0323. A.S gratefully acknowledges the financial support of the FP7-IDEAS-ERC-StG \#256872 (EntroPhase). Part of this work has been done while A.S. was visiting the Erwin Schrödinger Institute in Vienna for the ESI Thematic Program 'Nonlinear Flows'. G.C. and A.S. acknowledge the partial support of the GNAMPA (Gruppo Nazionale per l'Analisi Matematica, la Probabilità e le loro Applicazioni) group of INdAM (Istituto Nazionale di Alta Matematica). The authors would like to thank John M. Ball and Epifanio G. Virga, for inspiring conversations and interesting remarks. The authors also gratefully acknowledge the positive criticism from the referees.

Conflict of Interest. The authors of this manuscript declare that they have no conflict of interest.

\section{References}

1. R. Alicandro and M. Cicalese. Variational analysis of the asymptotics of the $X Y$ model. Arch. Ration. Mech. Anal., 192(3):501-536, 2009.

2. R. Alicandro, L. De Luca, A. Garroni, and M. Ponsiglione. Metastability and dynamics of discrete topological singularities in two dimensions: a $\Gamma$-convergence approach. Arch. Ration. Mech. Anal., 214(1):269-330, 2014.

3. R. Alicandro and M. Ponsiglione. Ginzburg-Landau functionals and renormalized energy: a revised $\Gamma$-convergence approach. J. Funct. Anal., 266(8):4890-4907, 2014.

4. N. Amenta, S. Choi, T. K. Dey, and N. Leekha. A simple algorithm for homeomorphic surface reconstruction. Int. J. Comput. Geom. Ap., 12(01n02):125-141, 2002.

5. Thierry Aubin. Some nonlinear problems in Riemannian geometry. Springer Monographs in Mathematics. Springer-Verlag, Berlin, 1998.

6. J. M. Ball. Convexity conditions and existence theorems in nonlinear elasticity. Arch. Rational Mech. Anal., 63(4):337-403, 1976/77.

7. J. M. Ball. Mathematics and liquid crystals. arXiv, abs/1612.0379v2, 2016.

8. J. M. Ball. Liquid crystals and their defects. arXiv, arXiv:1706.06861v3, 2017.

9. S. Baraket. Critical points of the Ginzburg-Landau system on a Riemannian surface. Asymptotic Anal., 13(3):277-317, 1996. 
10. S. Bartels. Finite Elements Approximation of Harmonic Maps between Surfaces. Habilitation thesis, Humboldt-Universität, Berlin, 2009

11. V. L. Berezinskii. Destruction of long-range order in one-dimensional and twodimensional systems possessing a continuous symmetry group. i. Classical systems. J. Exp. Theor. Phys., 61(3):1144, 1972.

12. F. Bethuel, H Brezis, and F Hélein. Ginzburg-Landau vortices. Progress in Nonlinear Differential Equations and their Applications, 13. Birkhäuser Boston, Inc., Boston, MA, 1994.

13. J. Bourgain, H. Brezis, and P. Mironescu. Lifting in Sobolev spaces. J. Anal. Math., $80: 37-86,2000$.

14. M. J. Bowick and L. Giomi. Two-dimensional matter: order, curvature and defects. Adv. Phys., 58(5):449-563, 2009.

15. M. J. Bowick, D. Nelson, and A. Travesset. Curvature-induced defect unbinding in toroidal geometries. Phys. Rev. E, 69:041102, Apr 2004.

16. A. Braides, M. Cicalese, and F. Solombrino. $Q$-Tensor Continuum Energies as Limits of Head-to-Tail Symmetric Spin Systems. SIAM J. Math. Anal., 47(4):2832-2867, 2015.

17. G. Canevari and A. Segatti. Variational analysis of nematic shells. In Trends on Applications of Mathematics to Mechanics, volume to appear. Springer-Indam Series, 2017.

18. G. Canevari, A. Segatti, and M. Veneroni. Morse's index formula in VMO for compact manifolds with boundary. J. Funct. Anal., 269(10):3043-3082, 2015.

19. P. G. Ciarlet. The finite element method for elliptic problems. North-Holland Publishing Co., Amsterdam-New York-Oxford, 1978. Studies in Mathematics and its Applications, Vol. 4.

20. A. Contreras and P. Sternberg. Gamma-convergence and the emergence of vortices for Ginzburg-Landau on thin shells and manifolds. Calc. Var. Partial Differential Equations, 38(1-2):243-274, 2010.

21. J. Dávila and R. Ignat. Lifting of BV functions with values in $S^{1}$. C. R. Math. Acad. Sci. Paris, 337(3):159-164, 2003.

22. Lucia De Luca. $\Gamma$-convergence analysis for discrete topological singularities: the anisotropic triangular lattice and the long range interaction energy. Asymptot. Anal., 96(3-4):185-221, 2016.

23. M. P. do Carmo. Riemannian geometry. Mathematics: Theory \& Applications. Birkhäuser Boston Inc., Boston, MA, 1992. Translated from the second Portuguese edition by Francis Flaherty.

24. K. Hildebrandt, K. Polthier, and M. Wardetzky. On the convergence of metric and geometric properties of polyhedral surfaces. Geom. Dedicata, 123:89-112, 2006.

25. R. Ignat and R Jerrard. Interaction energy between vortices of vector fields on riemannian surfaces. ArXiv: 1701.06546, 2017.

26. R. Ignat and R Jerrard. Renormalized energy between vortices in some ginzburg-landau models on riemannian surfaces. preprint, 2017.

27. R. L. Jerrard. Lower bounds for generalized Ginzburg-Landau functionals. SIAM J. Math. Anal., 30(4):721-746, 1999.

28. R. L. Jerrard and H. M. Soner. Rectifiability of the distributional Jacobian for a class of functions. C. R. Acad. Sci. Paris Sér. I Math., 329(8):683-688, 1999.

29. R. L. Jerrard and H. M. Soner. The Jacobian and the Ginzburg-Landau energy. Cal. Var. Partial Differential Equations, 14(2):151-191, 2002.

30. T. W. B. Kibble. Symmetry breaking and defects. In Patterns of Symmetry Breaking, volume 127 of NATO Science Series, pages 3-36. Springer, Berlin, 2003.

31. J. M. Kosterlitz and D. J. Thouless. Ordering, metastability and phase transitions in two-dimensional systems. Journal of Physics C: Solid State Physics, 6(7):1181, 1973.

32. John M. Lee. Introduction to smooth manifolds, volume 218 of Graduate Texts in Mathematics. Springer, New York, second edition, 2013.

33. T. C. Lubensky and J. Prost. Orientational order and vesicle shape. J. Phys. II France, $2(3): 371-382,1992$.

34. S. Müller. Det $=$ det. A remark on the distributional determinant. C. R. Acad. Sci. Paris Sér. I Math., 311(1):13-17, 1990.

35. M. Mutz and D. Bensimon. Observation of toroidal vesicles. Phys. Rev. A, 43:45254527, Apr 1991 
36. G. Napoli and L. Vergori. Extrinsic curvature effects on nematic shells. Phys. Rev. Lett., 108(20):207803, 2012.

37. G. Napoli and L. Vergori. Surface free energies for nematic shells. Phys. Rev. E, 85(6):061701, 2012.

38. D. R. Nelson. Toward a tetravalent chemistry of colloids. Nano Lett., 2(10):1125-1129, 2002.

39. J. Qing. Renormalized energy for Ginzburg-Landau vortices on closed surfaces. Math. Z., 225(1):1-34, 1997.

40. É. Sandier. Lower bounds for the energy of unit vector fields and applications. J. Funct. Anal., 152(2):379-403, 1998. see Erratum, ibidem 171, 1 (2000), 233.

41. É. Sandier and S. Serfaty. Vortices in the magnetic Ginzburg-Landau model. Progress in Nonlinear Differential Equations and their Applications, 70. Birkhäuser Boston, Inc., Boston, MA, 2007.

42. É. Sandier and S. Serfaty. Improved lower bounds for Ginzburg-Landau energies via mass displacement. Anal. PDE, 4(5):757-795, 2011.

43. Etienne Sandier. Ginzburg-Landau minimizers from $\mathbb{R}^{n+1}$ to $\mathbb{R}^{n}$ and minimal connections. Indiana Univ. Math. J., 50(4):1807-1844, 2001.

44. R. Schoen and K. Uhlenbeck. Boundary regularity and the Dirichlet problem for harmonic maps. J. Differential Geom., 18(2):253-268, 1983.

45. A. Segatti. Variational models for nematic shells. Lecture Notes for a $\mathrm{PhD}$ course at Universidad Autónoma de Madrid, 2015

46. A. Segatti, M. Snarski, and M. Veneroni. Equilibrium configurations of nematic liquid crystals on a torus. Phys. Rev. E, 90(1):012501, 2014.

47. A. Segatti, M. Snarski, and M. Veneroni. Analysis of a variational model for nematic shells. Math. Models Methods Appl. Sci., 26(10):1865-1918, 2016.

48. R. L. Selinger, A. Konya, A. Travesset, and J. V. Selinger. Monte Carlo studies of the XY model on two-dimensional curved surfaces. J.Phys.Chem B, 48:12989-13993, 2011.

49. J. R. Shewchuk. Delaunay refinement algorithms for triangular mesh generation. Comp. Geom. Theor. Appl., 22(1-3):21-74, 2002. 16th ACM Symposium on Computational Geometry.

50. J. P. Straley. Liquid crystals in two dimensions. Phys. Rev. A, 4(2):675-681, 1971.

51. E. G. Virga. Variational theories for liquid crystals, volume 8 of Applied Mathematics and Mathematical Computation. Chapman \& Hall, London, 1994.

52. V. Vitelli and D. Nelson. Nematic textures in spherical shells. Phys. Rev. E, 74(2):021711, 2006

53. V. Vitelli and D. R. Nelson. Defect generation and deconfinement on corrugated topographies. Phys. Rev. E, 70:051105, Nov 2004 